INTER NATIONAL MONETARY FUND
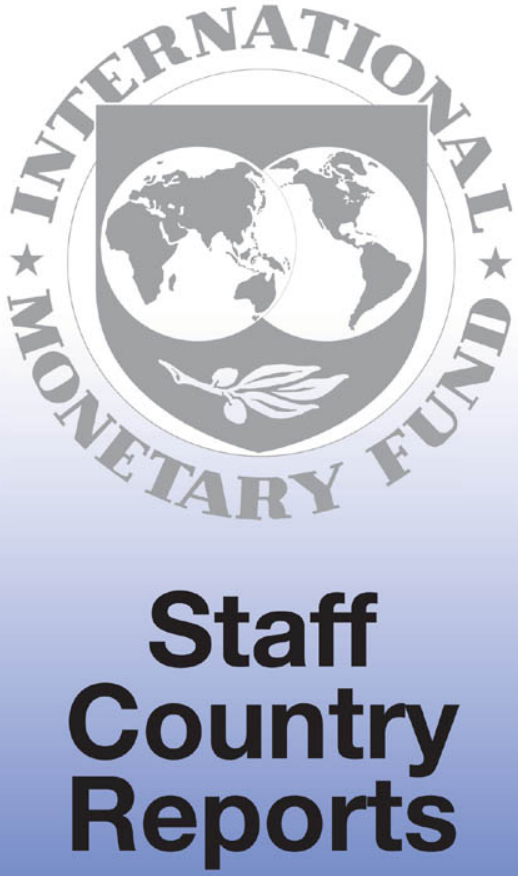
September 1999

IMF Staff Country Report No. 99/102

\section{Uruguay: Statistical Annex}

This Statistical Annex report on Uruguay was prepared by a staff team of the International Monetary Fund as background documentation for the periodic consultation with this member country. As such, the views expressed in this document are those of the staff team and do not necessarily reflect the views of the Government of Uruguay or the Executive Board of the IMF.

Copies of this report are available to the public from International Monetary Fund • Publication Services 700 19th Street, N.W. • Washington, D.C. 20431

Telephone: (202) 623-7430 • Telefax: (202) 623-7201

Telex (RCA): 248331 IMF UR

E-mail: publications@imf.org Internet: http://www.imf.org

Price: $\$ 15.00$ a copy

\section{International Monetary Fund Washington, D.C.}




\section{INTERNATIONAL MONETARY FUND}

\section{URUGUAY}

\section{Statistical Annex}

Prepared by B. Traa (Head) J. Bailén, B. Braumann, J.C. Jaramillo and

E. Tanner (all WHD)

Approved by the Western Hemisphere Department

July 14, 1999

Contents

Basic Data

Tables

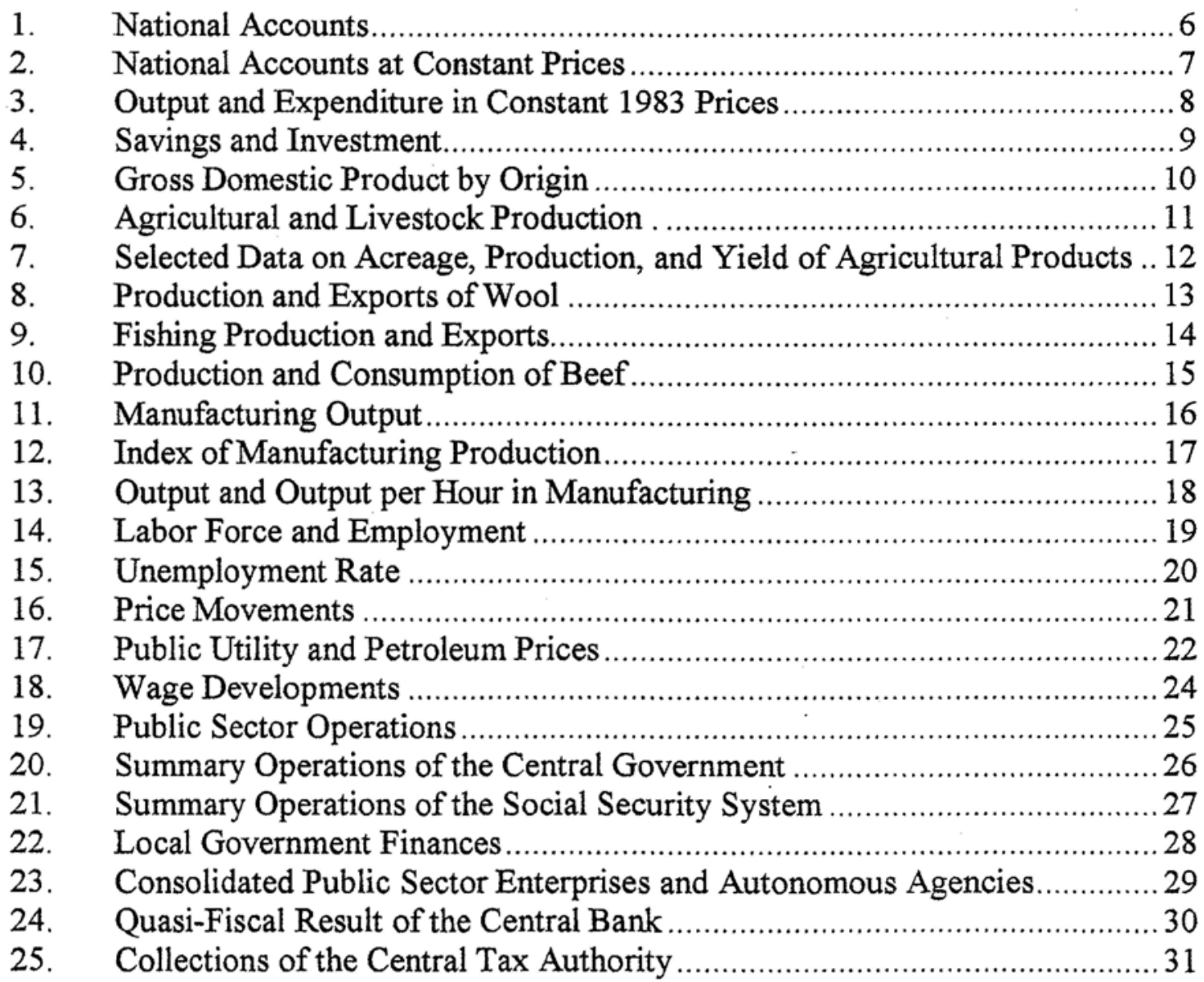

CInternational Monetary Fund. Not for Redistribution 
26. Operations of the Public Enterprises and Autonomous Agencies ................. 32

27. Functional Classification of Central Government Expenditure ....................36

28. Employment in the Public Sector, 1993-98 ….......................................... 37

29. Selected Monetary and Credit Indicators ……............................................... 38

30. Summary Accounts of the Financial System............................................. 39

31. Financial System Credit to the Private Sector …...................................... 40

32. Financial System Credit to the Private Sector in Real Terms ..................... 41

33. Composition of Private Sector Financial Assets ....................................... 42

34. Accounts of the Financial System........................................................... 43

35. Accounts of the Banking System ......................................................... 44

36. Accounts of the Central Bank ................................................................. 45

37. Accounts of the Banco de la República Oriental del Uruguay......................46

38. Accounts of the Private Commercial Banks...........................................47

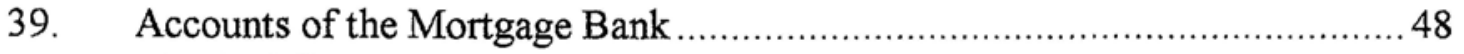

40. Nominal Short-Term Interest Rates ................................................... 49

41. Private Banks' Required Reserve Ratios on Selected Dates ......................50

42. $\quad$ Summary Balance of Payments ............................................................... 53

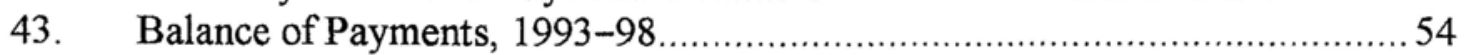

44. Value, Volume, and Unit Value ........................................................ 56

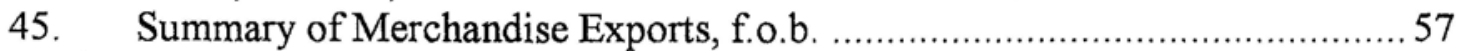

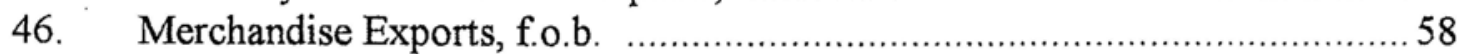

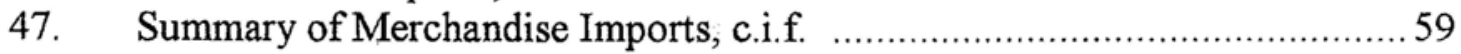

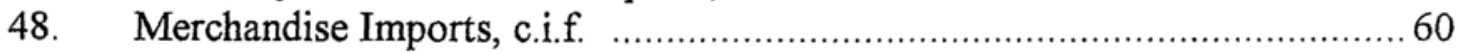

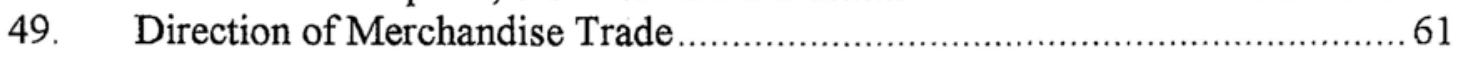

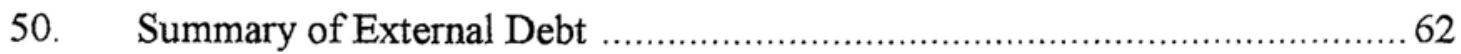

51. Summary of Public External Debt Service ..........................................63

52. Disbursements, Amortization, and Outstanding External Public Debt.........6 64

53. Amortization for Medium- and Long-Term Public Sector External Debt......68

54. Net International Reserves of the Central Bank ......................................69

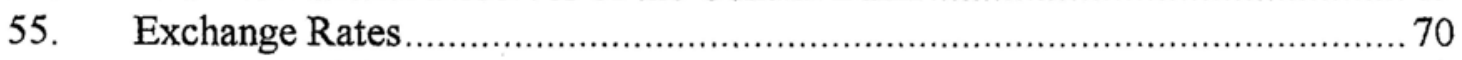

56. Measures of Competitiveness .............................................................. 71

\section{$\underline{\text { Appendices }}$}

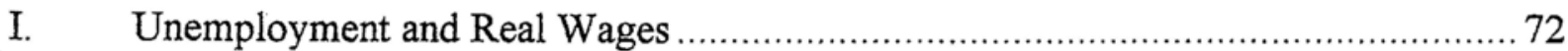

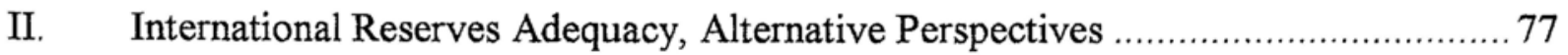

\section{Appendix Tables}

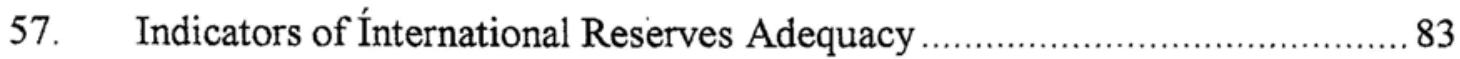

58. International Reserves and Yield Spread by Emerging Markets.................. 84 


\section{Appendix Figures}

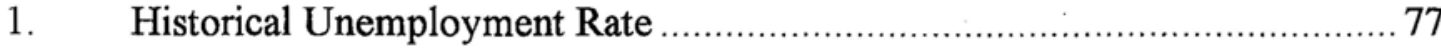

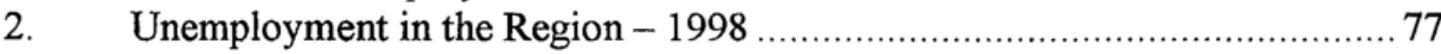

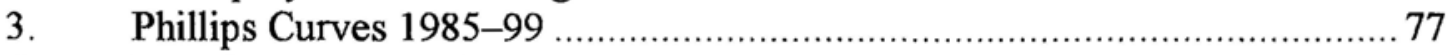

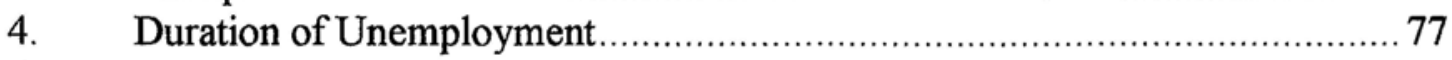

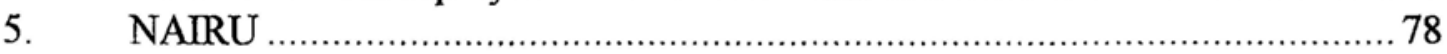

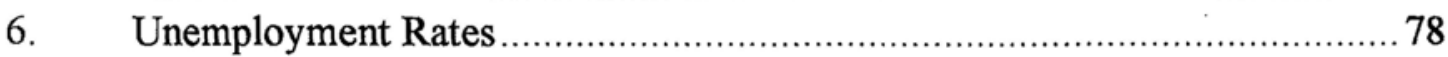

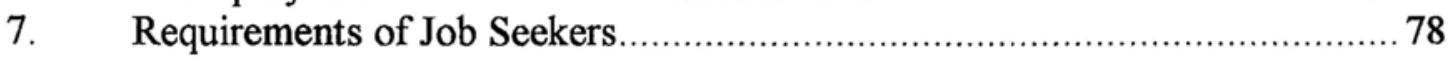

$8 . \quad$ Real Wages 1960-98.................................................................. 79

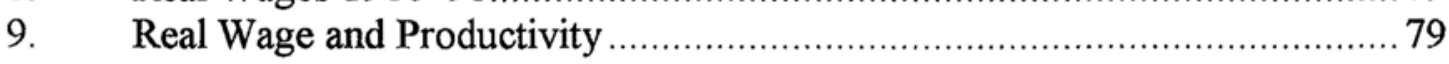

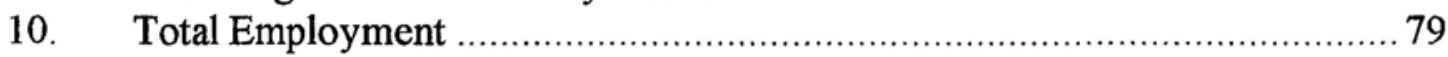

11. Real Wages and Household Income …................................................ 79

(CInternational Monetary Fund. Not for Redistribution 
Uruguay--Basic Data

Social and demographic indicators

Ares

Population (first quarter 1998, estimate)

Annual rate of population increase (1980-95)

Crude birth rate (1993)

Crude death rate (1993)

Infant mortality (1994)

Population per physician (1992)

Population per hospital bed (1992)

Urban population with access to safe water (1992)

Rural population with access to safe water (1992)

Energy consumption per capita (1994)

Per capita protein intake (1991)

Per capita intake of calories (1991)

Primary school enrollment (1993)

Unemployment rate (second quarter 1998)

GDP (1998)

GDP per capita (1998)
Central administration expenditure

Public sector savings (including losses of

official banks)

Nonfinancial public sector overall

surplus or deficit (-)

Financial public sector balance

Combined public sector balance

Combined public sector balance -excluding the cost of the reforms

External public debt (end of period) $1 /$

Total external debt (end of period) $1 /$

Total debt service ratio (in percent of

exports of goods and nonfactor services)

Public interest payments (in percent of

exports of goods and nonfactor services) $2 /$

National savings

Gross domestic investment

Money and quasi-money in

domestic currency (M2)

Annual changes in selected economic indicators

Real GDP per capita

Real GDP

GDP at current prices

Domestic expenditure (at current prices)

Investment

Consumption

GDP deflator

Wholesale prices (annual average)

Consumer prices (annual average)

Consumer prices (end of period)

Central administration expenditure 3/

Central administration current expenditure $3 /$

Central administration current expenditure

Money and quasi-money in domestic currency (M-2)

Money (M-1)

Quasi-money

Money and quasi-money in domestic and

foreign currency (M-3) 3/

Real M-2
1995

1996

21.1

21.3

$-1.2$

19.1

19.8

176,320 sq. kilometers 3.23 million

0.5 percent

17 per 1,000

10 per 1,000

19 per 1,000 live births

365$$
238
$$

95 percent

65 percent

623 kilograms of oil equivalent

79 grams per day

2,791 per day

109 percent

10.1 percent

Ur\$218.1 billion

US\$2 20.8 billion

US\$6,449

Prel.

1997

1998

21.9

22.5

$-1.9$

21.0

20.8

3.4

$-0.6$

$-0.5$

$-0.9$

0.4

26.7

39.4

14.1

9.7

10.1

28.0

16.5

8.9

13.9

15.8

7.9

7.1

7.3

7.5

5.7

$-2.4$

4.6

4.4

3.8

4.5

15.7

16.0

43.1

12.1

52.3

30.9 
Annual changes in selected economic indicators (continued)

Merchandise exports (f.o.b., in U.S. dollars)

Merchandise imports (f.o.b., in U.S. dollars)

Total extemal debt (in U.S. dollars)

External public debt (in U.S. dollars)

Terms of trade (deterioration -, period average)

Nominal effective exchange rate (depreciation - , end of period)

Real effective exchange rate (depreciation - , end of period)

\section{Central administration finances}

Revenue

Current expenditure 3/

Current account 3 /

Overall balance $3 /$

\section{Balance of payments}

Merchandise exports (f.o.b.)

Merchandise imports (f.o.b.)

Investment income (net)

Other services and transfers (net)

1,913

$-2,600$

Current account balance

Public sector capital (net)

Private sector capital (net),

including errors and omissions

Overall balance (deficit -)

Valuation adjustment 5/

Change in official net reserves (increase -)

\section{International reserve position and external debt}

Central Bank international reserves (gross)

(in months of imports) 6/

Financial system international reserves (net) $7 /$

Total external debt 8/

Public external debt

\section{IMF data (as of June 30, 1999)}

Membership status

Intervention currency and rate

Quots

Fund holdings of new Uruguayan pesos

As percent of quots

Reserve position in Fund

As percent of quota

Total Fund credit

From Fund resources

Credit tranche purchases (including SBA)

From supplementary and enlarged access resources

Stand-by purchases

Special Drawing Rights Department

Cumulative SDR allocation

Net acquisition or utilization (-) of SDRs

Holdings of SDRs

Share of profits from gold sales

$\begin{array}{rrrr}2,148 & 2,449 & 2,793 & 2,832 \\ -2,711 & -3,135 & -3,498 & -3,594 \\ -227 & -189 & -193 & -185 \\ 578 & 642 & 610 & 547\end{array}$

1/ Excluding reserve liabilities of the Central Bank and foreign currency deposits by nonresidents.

2/ On public and publicly guaranteed debt; excludes foreign currency deposits of nonresidents, reserve liabilities of the Central Bank, and short-term amortization.

3/ Excludes the costs of reforms (principally severance payments)

4/ Includes foreign currency deposits of residents and nonresidents; foreign currency deposits converted at the end-of-period exchange rate.

$5 /$ Includes an adjustment to allow for the difference between the price at which gold is bought or sold and the price at which it is valued for reserve purposes.

6/ Gold valued at market prices.

7/ Includes the Cental Bank of Uruguay, the Bank of the Republic, the private commercial banks, and the mortgage bank.

8/ Includes private suppliers credits and excludes reserve liabilities of the commercial banks and deposits of nonresidents. 
Table 1. Uruguay: National Accounts

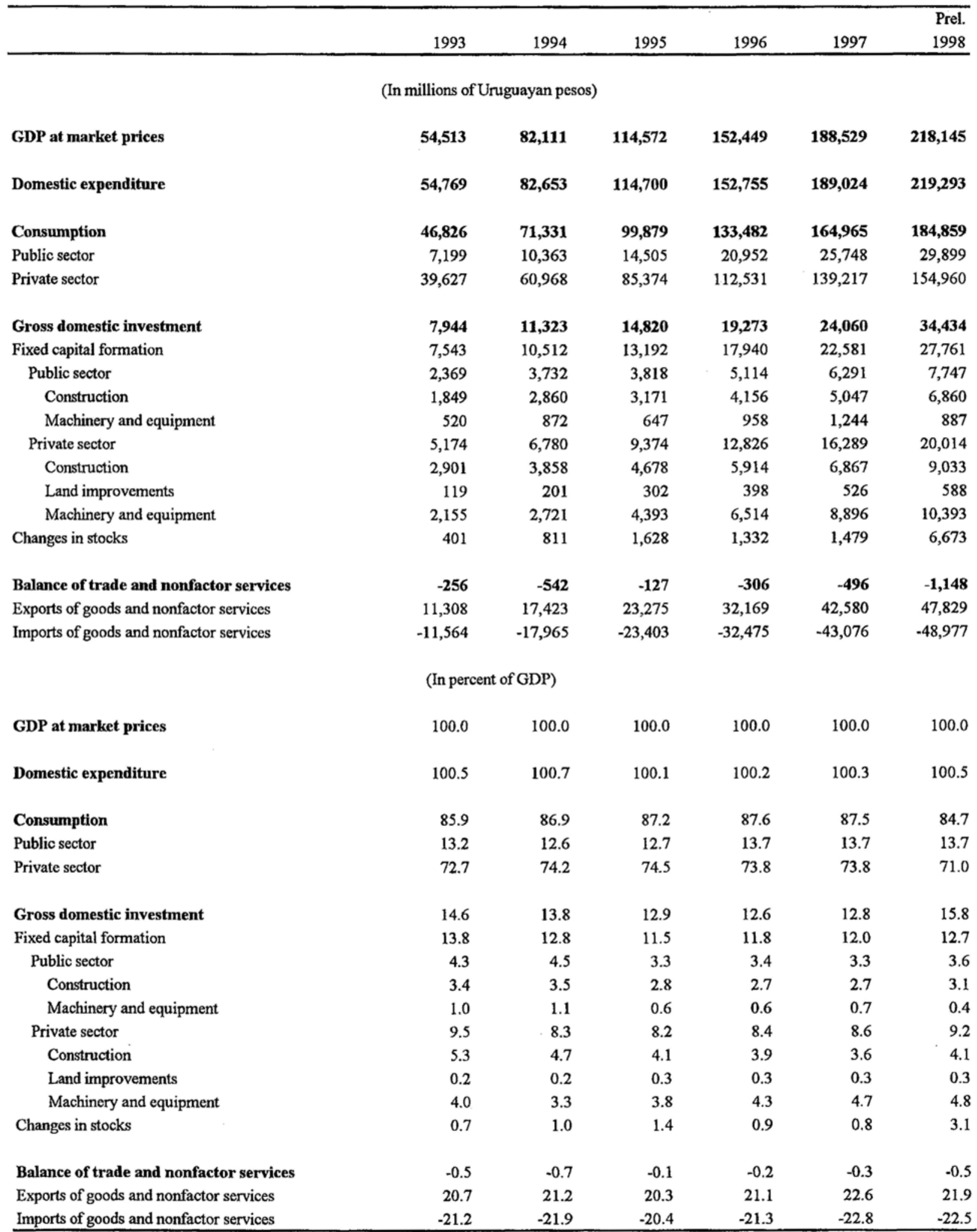

Source: Central Bank of Uruguay. 
Table 2. Uruguay: National Accounts at Constant Prices

(In millions of Uruguayan pesos at 1983 prices)

\begin{tabular}{|c|c|c|c|c|c|c|}
\hline & 1993 & 1994 & 1995 & 1996 & 1997 & $\begin{array}{c}\text { Prel. } \\
1998 \\
\end{array}$ \\
\hline GDP at market prices & 242.4 & 257.7 & 253.2 & 266.6 & 280.1 & 292.6 \\
\hline Domestic expenditure & 258.2 & 278.0 & 273.9 & 290.8 & 307.5 & 330.8 \\
\hline Consumption & 222.2 & 239.3 & 233.2 & 250.7 & 264.7 & 274.6 \\
\hline Public sector & 31.2 & 32.6 & 32.6 & 34.3 & 35.2 & 36.4 \\
\hline Private sector & 191.1 & 206.7 & 200.5 & 216.5 & 229.4 & 238.2 \\
\hline Gross domestic investment & 35.9 & 38.8 & 40.7 & 40.0 & 42.9 & 56.2 \\
\hline Fixed capital formation & 33.7 & 34.3 & 32.8 & 36.4 & 39.5 & 43.6 \\
\hline Public sector & 10.8 & 11.9 & 8.4 & 9.0 & 9.5 & 10.1 \\
\hline Construction & 7.6 & 8.1 & 6.3 & 6.5 & 6.9 & 8.2 \\
\hline Machinery and equipment & 3.1 & 3.7 & 2.1 & 2.5 & 2.6 & 1.9 \\
\hline Private sector & 23.0 & 22.5 & 24.4 & 27.4 & 30.0 & 33.5 \\
\hline Construction & 10.7 & 10.2 & 9.1 & 9.1 & 9.2 & 10.6 \\
\hline Land improvements & 0.7 & 0.9 & 1.1 & 1.1 & 1.2 & 1.2 \\
\hline Machinery and equipment & 11.5 & 11.3 & 14.1 & 17.1 & 19.6 & 21.7 \\
\hline Changes in inventories & 2.2 & 4.4 & 8.0 & 3.6 & 3.4 & 12.6 \\
\hline Balance of trade and nonfactor services & -15.8 & -20.3 & -20.7 & -24.2 & -27.5 & -38.2 \\
\hline Exports of goods and nonfactor services & 79.5 & 91.0 & 90.2 & 99.3 & 112.3 & 114.2 \\
\hline Imports of goods and nonfactor services & -95.3 & -111.3 & -110.9 & -123.5 & -139.8 & -152.4 \\
\hline
\end{tabular}

Source: Central Bank of Uruguay. 
Table 3. Uruguay: Output and Expenditure in Constant 1983 Prices

(Annual percentage change)

\begin{tabular}{|c|c|c|c|c|c|c|}
\hline & 1993 & 1994 & 1995 & 1996 & 1997 & $\begin{array}{l}\text { Prel. } \\
1998 \\
\end{array}$ \\
\hline Gross domestic product & 3.0 & 6.3 & -1.8 & 5.3 & 5.1 & 4.5 \\
\hline Domestic expenditure & 6.7 & 7.7 & -1.5 & 6.2 & 5.8 & 7.6 \\
\hline Consumption & 5.5 & 7.7 & -2.6 & 7.5 & 5.6 & 3.8 \\
\hline Public sector & 0.9 & 4.5 & 0.2 & 5.0 & 2.8 & 3.4 \\
\hline Private sector & 6.3 & 8.2 & -3.0 & 8.0 & 6.0 & 3.8 \\
\hline Gross domestic investment & 14.2 & 7.9 & 5.1 & -1.7 & 7.1 & 31.1 \\
\hline Fixed capital formation & 16.3 & 1.8 & -4.6 & 11.2 & 8.3 & 10.4 \\
\hline Public sector & 35.5 & 10.2 & -29.1 & 7.1 & 5.6 & 6.1 \\
\hline Private sector & 9.1 & -2.1 & 8.4 & 12.6 & 9.3 & 11.8 \\
\hline Change in stocks $1 /$ & -0.1 & 0.9 & 1.4 & -1.7 & -0.1 & 3.3 \\
\hline Balance of trade and nonfactor services & -3.8 & -1.9 & -0.2 & -1.4 & -1.2 & -3.8 \\
\hline Exports of goods and nonfactor services & 8.3 & 14.6 & -0.9 & 10.1 & 13.1 & 1.7 \\
\hline Imports of goods and nonfactor services & 18.8 & 16.8 & -0.4 & 11.4 & 13.2 & 9.0 \\
\hline
\end{tabular}

Source: Central Bank of Uruguay.

1/ Contribution to GDP growth. 
Table 4. Uruguay: Saving and Investment

(As percent of GDP at current market prices)

\begin{tabular}{|c|c|c|c|c|c|c|}
\hline & 1993 & 1994 & 1995 & 1996 & 1997 & $\begin{array}{c}\text { Prel. } \\
1998 \\
\end{array}$ \\
\hline GDP at market prices & 100.0 & 100.0 & 100.0 & 100.0 & 100.0 & 100.0 \\
\hline Domestic expenditure & 100.5 & 100.7 & 100.1 & 100.2 & 100.3 & 100.5 \\
\hline Consumption & 85.9 & 86.9 & 87.2 & 87.6 & 87.5 & 84.7 \\
\hline Public sector & 13.2 & 12.6 & 12.7 & 13.7 & 13.7 & 13.7 \\
\hline Private sector & 72.7 & 74.2 & 74.5 & 73.8 & 73.8 & 71.0 \\
\hline Gross domestic investment & 14.6 & 13.8 & 12.9 & 12.6 & 12.8 & 15.8 \\
\hline Fixed capital formation & 13.8 & 12.8 & 11.5 & 11.8 & 12.0 & 12.7 \\
\hline Public sector & 4.3 & 4.5 & 3.3 & 3.4 & 3.3 & 3.6 \\
\hline Private sector & 9.5 & 8.3 & 8.2 & 8.4 & 8.6 & 9.2 \\
\hline Changes in stocks & 0.7 & 1.0 & 1.4 & 0.9 & 0.8 & 3.1 \\
\hline Gross national savings & 12.0 & 11.1 & 11.8 & 11.4 & 11.3 & 13.9 \\
\hline Public sector 1/ & 3.0 & 2.5 & 2.6 & 2.0 & 2.4 & 2.6 \\
\hline Private sector & 9.0 & 8.6 & 9.2 & 9.4 & 8.9 & 11.3 \\
\hline Current account balance & -2.6 & -2.7 & -1.2 & -1.2 & -1.4 & -1.9 \\
\hline
\end{tabular}

Sources: Central Bank of Uruguay; and Fund staff estimates.

1/ Public sector total revenue minus current expenditure; private saving is residual. 
Table 5. Uruguay: Gross Domestic Product by Origin

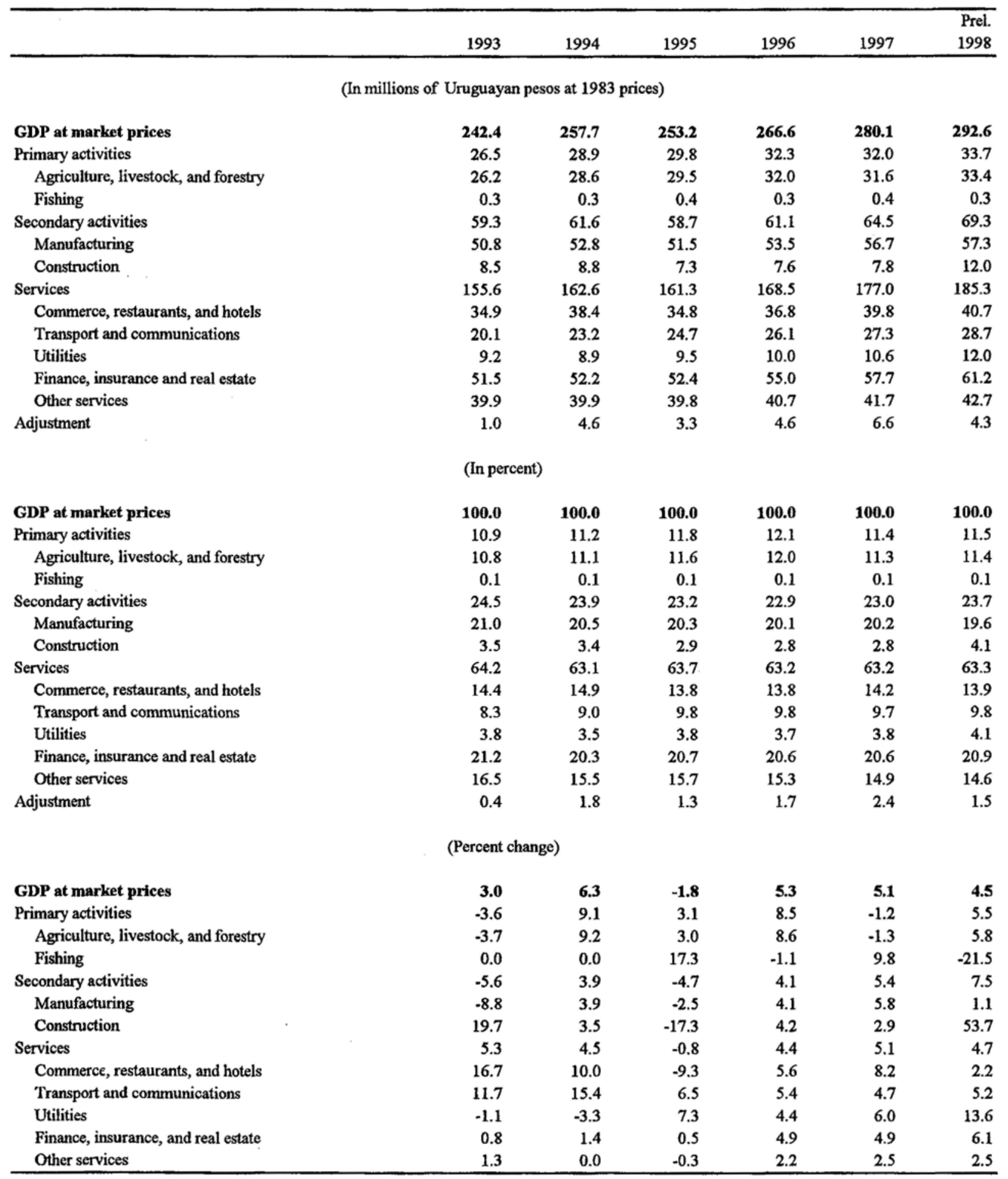

Source: Central Bank of Uruguay.

1/ Includes mining and quarrying.

2/ Includes commercial and personal services.

3/ Subtracts imputed earnings of financial intermediaries and adds import duties. 
Table 6. Uruguay: Agricultural and Livestock Production 1/

(Percentage change)

\begin{tabular}{lrrrrrr}
\hline & 1993 & 1994 & 1995 & 1996 & 1997 & 1998 \\
\hline Total agriculture and livestock & $\mathbf{- 2 . 8}$ & $\mathbf{7 . 4}$ & $\mathbf{3 . 8}$ & $\mathbf{9 . 8}$ & $\mathbf{- 1 . 7}$ & $\mathbf{5 . 8}$ \\
Agricultural products & & & & & & \\
Cereals & $\mathbf{- 9 . 5}$ & $\mathbf{1 6 . 1}$ & $\mathbf{1 0 . 0}$ & $\mathbf{1 5 . 2}$ & $\mathbf{- 2 . 9}$ & $\mathbf{9 . 2}$ \\
Of which: & -12.0 & 22.4 & 12.9 & 22.0 & $\ldots$ & $\ldots$ \\
$\quad$ Wheat & & & & & & \\
$\quad$ Corn & 3.6 & 38.8 & -15.9 & 44.3 & $\ldots$ & $\ldots$ \\
$\quad$ Rice & -28.3 & 15.2 & 21.1 & 38.0 & $\ldots$ & $\ldots$ \\
Oilseed crops & 0.5 & 10.3 & 22.6 & 4.6 & $\ldots$ & $\ldots$ \\
Wine grapes & 11.3 & 44.2 & 8.1 & 2.4 & $\ldots$ & $\ldots$ \\
Other & -21.5 & 1.9 & 14.5 & 7.7 & $\ldots$ & $\ldots$ \\
Livestock products & 3.3 & 10.9 & 10.0 & 15.2 & $\ldots$ & $\ldots$ \\
Beef & & & & & & $\ldots$ \\
Other livestock & $\mathbf{2 . 6}$ & $\mathbf{1 . 2}$ & $\mathbf{- 1 . 2}$ & $\mathbf{5 . 0}$ & $\mathbf{- 0 . 5}$ & $\mathbf{2 . 8}$ \\
Wool & 4.8 & 0.8 & 0.8 & 6.9 & $\ldots$ & $\ldots$ \\
Milk & -8.9 & 7.4 & 12.7 & -8.8 & $\ldots$ & $\ldots$ \\
Other & 2.3 & -9.2 & -7.0 & 7.8 & $\ldots$ & $\ldots$ \\
\hline Source: Centra. & 3.9 & 6.2 & 5.3 & 3.8 & $\ldots$ & $\ldots$ \\
\hline & 0.3 & 24.3 & -14.5 & 3.1 & $\ldots$ & $\ldots$ \\
\hline
\end{tabular}

Source: Central Bank of Uruguay.

1/ Calendar year estimates. 
Table 7. Uruguay: Selected Data on Acreage, Production, and Yield of Agricultural Products

(Production in thousand metric tons; acreage in thousand hectares; yield in metric tons per hectare)

\begin{tabular}{|c|c|c|c|c|c|c|}
\hline & 1993 & 1994 & 1995 & 1996 & 1997 & $\begin{array}{r}\text { Prel. } \\
1998 \\
\end{array}$ \\
\hline & & & Cereals & & & \\
\hline \multicolumn{7}{|l|}{ Wheat } \\
\hline Acreage & 148.0 & 213.8 & 189.0 & 168.2 & 250.3 & 245.4 \\
\hline Production & 340.9 & 342.5 & 485.0 & 392.9 & 649.7 & 504.8 \\
\hline Yield & 2.303 & 1.602 & 2.566 & 2.336 & 2.596 & 2.057 \\
\hline \multicolumn{7}{|l|}{ Corn } \\
\hline Acreage & 65.3 & 51.0 & 44.2 & 59.0 & 61.3 & 60.3 \\
\hline Production & 128.3 & 83.2 & 108.4 & 128.1 & 162.1 & 203.3 \\
\hline Yield & 1.965 & 1.631 & 2.452 & 2.171 & 2.644 & 3.371 \\
\hline \multicolumn{7}{|l|}{ Rice } \\
\hline Acreage & 130.0 & 134.3 & 146.2 & 150.0 & 155.0 & 170.0 \\
\hline Production & 650.0 & 620.1 & 804.1 & 967.5 & 1034.7 & 874.0 \\
\hline Yield & 5.000 & 4.617 & 5.500 & 6.450 & 6.675 & 5.141 \\
\hline \multicolumn{7}{|l|}{ Barley } \\
\hline Acreage & 124.4 & 88.1 & 73.0 & 130.9 & 143.1 & 118.9 \\
\hline Production & 306.9 & 129.0 & 177.0 & 329.0 & 340.6 & 198.6 \\
\hline Yield & 2.467 & 1.464 & 2.425 & 2.513 & 2.380 & 1.670 \\
\hline \multicolumn{7}{|l|}{ Sorghum } \\
\hline Acreage & 40.5 & 25.7 & 42.6 & 32.9 & 38.8 & 30 \\
\hline Production & 129.5 & 63.1 & 135.8 & 92.1 & 129.7 & 91.1 \\
\hline \multirow[t]{2}{*}{ Yield } & 3.198 & 2.455 & 3.188 & 2.799 & 3.343 & 3.037 \\
\hline & \multicolumn{5}{|c|}{ Oilseeds and industrial crops } & \\
\hline \multicolumn{7}{|l|}{ Linseed } \\
\hline Acreage & 2.7 & 2.5 & 0 & 0 & 0 & 0 \\
\hline Production & 2.4 & 2.1 & 0.0 & 0.0 & 0.0 & 0.0 \\
\hline Yield & 0.889 & 0.840 & 0.000 & 0.000 & 0.000 & 0.000 \\
\hline \multicolumn{7}{|c|}{ Sunflower seed } \\
\hline Acreage & 57.6 & 60.8 & 106.3 & 91.6 & 96.8 & 81 \\
\hline Production & 52.4 & 61.6 & 119.6 & 112.3 & 114 & 78.5 \\
\hline Yield & 0.910 & 1.013 & 1.125 & 1.226 & 1.178 & 0.969 \\
\hline \multicolumn{7}{|l|}{ Sugarbeet } \\
\hline Acreage & 0.9 & 0 & 0 & 0 & 0 & 0 \\
\hline Production & 32 & 0 & 0 & 0 & 0 & 0 \\
\hline Yield & 35.556 & 0.000 & 0.000 & 0.000 & 0.000 & 0.000 \\
\hline \multicolumn{7}{|l|}{ Soybeans } \\
\hline Acreage & 8.8 & 12 & 7.5 & 7.5 & 8 & 9 \\
\hline Production & 17.5 & 22.5 & 14.0 & 14.0 & 14.0 & 16.8 \\
\hline Yield & 1.989 & 1.875 & 1.867 & 1.867 & 1.750 & 1.867 \\
\hline \multicolumn{7}{|l|}{ Sugarcane } \\
\hline Acreage & 6.6 & 3.8 & 3.7 & 3.7 & 3.4 & $\ldots$ \\
\hline Production & 303.6 & 205 & 181.2 & 188.4 & 194.6 & $\ldots$ \\
\hline Yield & 46.000 & 53.947 & 48.973 & 50.919 & 57.235 & $\ldots$ \\
\hline
\end{tabular}

Sources: Central Bank of Uruguay; and Ministry of Agriculture and Fishing. 
Table 8. Uruguay: Production and Exports of Wool 1/

(In thousands of metric tons)

\begin{tabular}{lrrrrrr}
\hline & & & & & & Prel. \\
& 1993 & 1994 & 1995 & 1996 & 1997 & 1998 \\
\hline Supply & $\mathbf{9 3 . 3}$ & $\mathbf{9 1 . 3}$ & $\mathbf{9 2 . 4}$ & $\mathbf{9 6 . 4}$ & $\mathbf{8 5 . 4}$ & $\mathbf{7 9 . 8}$ \\
Production & $\mathbf{8 9 . 9}$ & 81.6 & 75.9 & 81.8 & 75.5 & 67.7 \\
Imports & 3.4 & 9.7 & 16.5 & 14.6 & 9.9 & 12.1 \\
& & & & & & \\
Demand & $\mathbf{1 0 6 . 9}$ & $\mathbf{1 0 4 . 1}$ & $\mathbf{7 8 . 1}$ & $\mathbf{1 0 3 . 9}$ & $\mathbf{9 7 . 5}$ & $\mathbf{6 6 . 3}$ \\
Domestic & 3.0 & 3.0 & 3.0 & 3.0 & 3.0 & 3.0 \\
Exports & 103.9 & 101.1 & 75.1 & 100.9 & 94.5 & 63.3 \\
& & & & & & \\
Change in stocks & -13.6 & -12.8 & 14.3 & -7.5 & -12.1 & $\mathbf{1 3 . 5}$ \\
\hline
\end{tabular}

Source: Central Bank of Uruguay.

1/ Greasy wool equivalent. 
Table 9. Uruguay: Fishing Production and Exports

\begin{tabular}{|c|c|c|c|}
\hline & \multirow{2}{*}{$\begin{array}{r}\text { Tonnage } \\
\text { Caught } \\
\end{array}$} & \multicolumn{2}{|c|}{ Exports } \\
\hline & & Tons & Millions of US\$ \\
\hline 1993 & 113,320 & 65,728 & 73.5 \\
\hline 1994 & 120,537 & 64,387 & 74.4 \\
\hline 1995 & 126,120 & 65,350 & 82.2 \\
\hline 1996 & 123,239 & 68,597 & 84.5 \\
\hline 1997 & 133,212 & 80,035 & 92.5 \\
\hline 1998 & $\cdots$ & $\cdots$ & $\cdots$ \\
\hline \multicolumn{4}{|l|}{1996} \\
\hline First quarter & 21,780 & 16,285 & 16.4 \\
\hline Second quarter & 33,604 & 14,021 & 18.8 \\
\hline Third quarter & 36,824 & 20,927 & 25.4 \\
\hline Fourth quarter & 31,031 & 17,364 & 23.9 \\
\hline \multicolumn{4}{|l|}{1997} \\
\hline First quarter & 25,107 & 11,317 & 14.2 \\
\hline Second quarter & 39,777 & 22,760 & 23.5 \\
\hline Third quarter & 36,039 & 22,607 & 24.1 \\
\hline Fourth quarter & 32,289 & 23,351 & 30.7 \\
\hline \multicolumn{4}{|l|}{1998 (Prel.) } \\
\hline First quarter & 24,897 & 13,028 & 19.9 \\
\hline Second quarter & 32,179 & 16,363 & 23.9 \\
\hline Third quarter & 38,440 & 12,010 & 21.1 \\
\hline Fourth quarter & 29,645 & $\cdots$ & $\ldots$ \\
\hline
\end{tabular}

Source: Central Bank of Uruguay. 
Table 10. Uruguay: Production and Consumption of Beef

\begin{tabular}{|c|c|c|c|c|}
\hline & \multicolumn{2}{|c|}{ Production } & \multirow[b]{2}{*}{$\begin{array}{c}\text { Exports } \\
\text { (Metric tons) }\end{array}$} & \multirow[b]{2}{*}{$\begin{array}{l}\text { Consumption } \\
\text { (Metric tons) }\end{array}$} \\
\hline & $\begin{array}{l}\text { Av. Weight } \\
(\mathrm{Kgs} / \mathrm{Head})\end{array}$ & $\begin{array}{c}\text { Heads } \\
\text { (Thousands) }\end{array}$ & & \\
\hline 1990 & 490 & 1,559 & 185,454 & 149,098 \\
\hline 1991 & 502 & 1,260 & 108,985 & 187,461 \\
\hline 1992 & 506 & 1,334 & 109,828 & 218,789 \\
\hline 1993 & 495 & 1,377 & 96,601 & 213,062 \\
\hline 1994 & 484 & 1,612 & 146,209 & 215,190 \\
\hline 1995 & 476 & 1,510 & 138,498 & 199,087 \\
\hline 1996 & 483 & 1,840 & 205,361 & 201,600 \\
\hline 1997 & 476 & 2,115 & 263,264 & 197,600 \\
\hline 1998 & 473 & 2,045 & 250,268 & 192,700 \\
\hline \multicolumn{5}{|l|}{1996} \\
\hline First quarter & 488 & $\ldots$ & 45,684 & $\ldots$ \\
\hline Second quarter & 491 & $\ldots$ & 53,879 & $\ldots$ \\
\hline Third quarter & 476 & $\ldots$ & 54,233 & $\ldots$ \\
\hline Fourth quarter & 477 & $\ldots$ & 51,565 & $\ldots$ \\
\hline \multicolumn{5}{|l|}{1997} \\
\hline First quarter & 489 & $\ldots$ & 63,613 & $\ldots$ \\
\hline Second quarter & 486 & $\ldots$ & 75,609 & $\ldots$ \\
\hline Third quarter & 456 & $\ldots$ & 66,907 & $\ldots$ \\
\hline Fourth quarter & 469 & $\ldots$ & 57,135 & $\ldots$ \\
\hline \multicolumn{5}{|l|}{1998} \\
\hline First quarter & 485 & $\ldots$ & 67,671 & $\ldots$ \\
\hline Second quarter & 481 & $\ldots$ & 70,730 & $\ldots$ \\
\hline Third quarter & 454 & $\ldots$ & 56,739 & $\ldots$ \\
\hline Fourth quarter & 469 & $\ldots$ & 55,128 & $\ldots$ \\
\hline
\end{tabular}

Source: Central Bank of Uruguay. 
Table 11. Uruguay: Manufacturing Output

(Percentage change)

\begin{tabular}{lrrrrr}
\hline & 1993 & 1994 & 1995 & 1996 & 1997 \\
\hline & & & & & \\
& & & & & \\
Total manufacturing output & $\mathbf{8 . 9}$ & $\mathbf{4 . 0}$ & $\mathbf{- 2 . 8}$ & $\mathbf{4 . 0}$ & $\mathbf{5 . 8}$ \\
$\quad$ Of which: & & & & & \\
$\quad$ Food processing & -6.6 & 9.6 & 3.0 & 9.6 & 5.4 \\
Electrical machinery & -8.9 & 3.6 & -2.0 & 24.3 & 21.6 \\
Transportation equipment & -3.6 & 34.4 & -49.8 & -54.5 & 63.1 \\
Petroleum derivatives 1/ & -67.0 & -93.1 & $4,440.9$ & 16.2 & -5.4 \\
Textiles & -10.0 & 0.0 & -19.4 & -2.0 & 5.9 \\
Chemicals & -4.4 & 6.7 & -9.2 & -0.5 & -4.3 \\
Beverages & 2.7 & 0.2 & -1.3 & -1.8 & 9.6 \\
\hline
\end{tabular}

Source: Central Bank of Uruguay.

1/ 1993 and 1994 relfects the closure of the oil refinery for refurbishment, production resumed in 1995. 
Table 12. Uruguay: Index of Manufacturing Production

(Physical output; quartenty average 1983=100)

\begin{tabular}{|c|c|c|c|c|c|c|c|c|c|c|c|c|c|c|}
\hline & \multirow[b]{2}{*}{1993} & \multirow[b]{2}{*}{1994} & \multirow[b]{2}{*}{1995} & \multirow[b]{2}{*}{1996} & \multirow[b]{2}{*}{1997} & \multirow[b]{2}{*}{ i 998} & \multicolumn{4}{|c|}{1997} & \multicolumn{4}{|c|}{1998} \\
\hline & & & & & & & I & II & III & IV & I & II & III & IV \\
\hline & & & · & & & & & & & & & & & \\
\hline Food & 100.0 & 109.6 & 112.9 & 123.7 & 130.4 & 131.9 & 122.8 & 132.8 & 134.4 & 131.7 & 124.2 & 132.3 & 137.3 & 133.9 \\
\hline Beverages & 131.5 & 131.8 & 130.1 & 127.7 & 140.0 & 138.4 & 145.7 & 128.3 & 112.3 & 173.8 & 136.6 & 113.2 & 126.4 & 177.6 \\
\hline Tobacco & 135.1 & 134.7 & 120.6 & 134.0 & 147.3 & 167.0 & 123.3 & 152.2 & 158.6 & 155.3 & 161.1 & 149.6 & 176.9 & 180.5 \\
\hline Textiles & 129.2 & 129.2 & 104.1 & 102.0 & 108.0 & 87.8 & 106.5 & 114.8 & 108.7 & 101.8 & 98.6 & 97.9 & 82.0 & 72.7 \\
\hline Clothing & 97.6 & 88.0 & 76.6 & 79.1 & 89.3 & 75.6 & 72.4 & 112.6 & 90.5 & 81.6 & 79.5 & 86.6 & 63.8 & 72.5 \\
\hline Leather and leather products & 62.0 & 75.4 & 73.5 & 88.9 & 76.3 & 74.2 & 70.4 & 78.7 & 79.0 & 77.1 & 74.0 & 78.3 & 76.5 & 68.1 \\
\hline Shoes & 119.4 & 128.3 & 100.3 & 118.2 & 104.6 & 46.8 & 68.9 & 157.7 & 65.8 & 126.0 & 39.3 & 63.2 & 47.8 & 36.8 \\
\hline Paper and paper products & 150.4 & 163.3 & 138.4 & 173.1 & 195.0 & 207.0 & 183.0 & 196.0 & 209.4 & 191.7 & 191.8 & 207.8 & 214.2 & 214.0 \\
\hline Printing & 111.4 & 107.4 & 97.3 & 102.1 & 109.2 & 125.6 & 94.5 & 104.7 & 110.2 & 127.4 & 116.3 & 124.1 & 120.4 & 141.3 \\
\hline Chemicals & 131.8 & 140.6 & 127.6 & 127.0 & 121.5 & 125.8 & 106.3 & 120.6 & 134.9 & 124.1 & 110.8 & 111.2 & 141.4 & 139.8 \\
\hline Oil refining & 31.9 & 2.2 & 99.9 & 116.1 & 109.8 & 139.6 & 44.1 & 133.8 & 124.6 & 136.6 & 136.9 & 128.6 & 147.2 & 145.7 \\
\hline Rubber & 142.5 & 144.6 & 149.8 & 127.9 & 152.5 & 116.2 & 148.7 & 168.1 & 157.7 & 135.5 & 114.1 & 141.0 & 139.2 & 70.4 \\
\hline Plastics & 104.6 & 116.9 & 125.4 & 119.5 & 142.1 & 149.3 & 118.9 & 123.5 & 141.1 & 185.0 & 151.7 & 127.8 & 144.9 & 172.8 \\
\hline Clay and porcelain & 463.4 & 530.7 & 473.8 & 450.0 & 454.1 & 369.7 & 479.6 & 435.5 & 435.7 & 465.5 & 363.7 & 378.7 & 305.1 & 431.3 \\
\hline Glass & 169.8 & 112.7 & 92.6 & 84.8 & 92.4 & 78.7 & 99.3 & 104.5 & 72.1 & 93.6 & 90.7 & 42.0 & 77.5 & 104.4 \\
\hline Other nonmetallic mineral products & 115.8 & 128.1 & 104.5 & 105.6 & 124.4 & 128.2 & 111.0 & 116.4 & 126.8 & 143.4 & 125.5 & 115.8 & 135.6 & 135.6 \\
\hline Metallics & 101.2 & 92.7 & 87.8 & 86.1 & 94.0 & 98.0 & 53.8 & 104.4 & 105.0 & 112.6 & 64.0 & 106.5 & 111.1 & 110.3 \\
\hline Machinery and metallic products & 109.1 & 123.1 & 90.2 & 84.6 & 80.9 & 71.0 & 64.9 & 89.5 & 90.9 & 78.2 & 69.0 & 73.2 & 66.4 & 75.5 \\
\hline Machinery and electric products & 139.6 & 144.6 & 141.7 & 176.2 & 214.2 & 227.8 & 201.4 & 220.1 & 221.9 & 213.4 & 189.0 & 219.7 & 277.6 & 232.7 \\
\hline Transportation materials & 314.2 & 422.3 & 211.9 & 96.5 & 157.4 & 313.5 & 56.6 & 134.3 & 202.6 & 236.2 & 228.4 & 326.1 & 373.1 & 326.3 \\
\hline Other manufacturing & 90.2 & 94.9 & 89.9 & 92.5 & 98.7 & 102.6 & 85.5 & 101.7 & 102.0 & 105.4 & 96.2 & 99.6 & 107.1 & 107.3 \\
\hline Total & 113.2 & 117.7 & 114.4 & 119.0 & 125.9 & 128.9 & 110.1 & 130.1 & 129.5 & 134.0 & 122.2 & 124.9 & 133.4 & 135.0 \\
\hline Year-on-year percentage change & -8.9 & 4.0 & -2.8 & 4.0 & 5.8 & 2.4 & -3.8 & 14.6 & 5.9 & 4.6 & 11.0 & -4.0 & 3.0 & 0.7 \\
\hline
\end{tabular}


Table 13. Uruguay: Output and Output per Hour in Manufacturing

(Percentage change over corresponding period of previous year)

\begin{tabular}{|c|c|c|c|}
\hline & $\begin{array}{r}\text { Manufacturing } \\
\text { Output } \\
\end{array}$ & $\begin{array}{r}\text { Hours } \\
\text { Worked } \\
\end{array}$ & $\begin{array}{r}\text { Output } \\
\text { per Hour }\end{array}$ \\
\hline 1993 & -11.7 & -5.3 & -6.1 \\
\hline 1994 & 2.9 & -5.7 & 9.1 \\
\hline 1995 & 3.1 & -10.1 & 14.8 \\
\hline 1996 & 4.8 & -4.5 & 9.7 \\
\hline 1997 & 4.9 & -0.1 & 5.0 \\
\hline \multicolumn{4}{|l|}{1998} \\
\hline \multicolumn{4}{|l|}{1993} \\
\hline 1st quarter & -2.7 & -9.8 & 7.9 \\
\hline 2nd quarter & -16.1 & -14.9 & -1.5 \\
\hline 3rd quarter & -16.6 & 2.3 & -18.5 \\
\hline 4th quarter & -10.9 & 1.4 & -12.1 \\
\hline \multicolumn{4}{|l|}{1994} \\
\hline 1st quarter & -14.0 & -11.4 & -2.8 \\
\hline 2nd quarter & 9.9 & -5.4 & 16.2 \\
\hline 3 rd quarter & 9.3 & -1.8 & 11.3 \\
\hline 4th quarter & 7.5 & -3.7 & 11.6 \\
\hline \multicolumn{4}{|l|}{1995} \\
\hline 1st quarter & 8.6 & -3.4 & 12.4 \\
\hline 2nd quarter & 6.1 & -9.2 & 16.9 \\
\hline 3rd quarter & -4.9 & -14.4 & 11.2 \\
\hline 4th quarter & 3.6 & -13.1 & 19.2 \\
\hline \multicolumn{4}{|l|}{1996} \\
\hline 1st quarter & 10.6 & -11.0 & 24.2 \\
\hline 2nd quarter & -5.3 & -7.9 & 2.9 \\
\hline 3rd quarter & 11.9 & -0.3 & 12.2 \\
\hline 4th quarter & 3.3 & 1.7 & 1.6 \\
\hline \multicolumn{4}{|l|}{1997} \\
\hline 1st quarter & -8.7 & -1.0 & -7.8 \\
\hline 2nd quarter & 14.9 & 2.6 & 12.0 \\
\hline 3rd quarter & 5.6 & 0.2 & 5.3 \\
\hline 4th quarter & 7.8 & -2.2 & 10.3 \\
\hline \multicolumn{4}{|l|}{1998 (Prel.) } \\
\hline 1st quarter & 18.9 & 0.0 & 18.9 \\
\hline 2nd quarter & -2.0 & -7.9 & 6.4 \\
\hline 3rd quarter & 5.8 & -7.9 & 14.9 \\
\hline 4th quarter & 1.8 & -7.4 & 9.9 \\
\hline
\end{tabular}

Sources: National Institute of Statistics; and Central Bank of Uruguay. 
Table 14. Uruguay: Labor Force and Employment

(As a percentage of population 14 years of age and older)

\begin{tabular}{|c|c|c|c|c|c|c|}
\hline & & Labor I & & & ployment & \\
\hline & Male & Female & Total & Male & Female & Total \\
\hline 1990 & & & & & & \\
\hline First half & 74.6 & 48.3 & 60.0 & 69.7 & 42.6 & 54.7 \\
\hline Second half & 74.4 & 46.5 & 59.1 & 68.5 & 41.0 & 53.4 \\
\hline 1991 & & & & & & \\
\hline First half & 73.6 & 46.7 & 58.8 & 68.1 & 40.9 & 53.2 \\
\hline Second half & 75.0 & 48.2 & 60.2 & 69.9 & 43.3 & 55.3 \\
\hline 1992 & & & & & & \\
\hline First half & 73.2 & 47.5 & 59.0 & 68.1 & 41.4 & 53.2 \\
\hline Second half & 73.4 & 49.3 & 60.0 & 68.8 & 44.0 & 55.0 \\
\hline 1993 & & & & & & \\
\hline First half & 73.4 & 48.0 & 59.2 & 68.7 & 42.3 & 54.0 \\
\hline Second half & 72.0 & 48.1 & 58.7 & 67.5 & 43.1 & 54.1 \\
\hline 1994 & & & & & & \\
\hline First half & 73.8 & 48.0 & 59.7 & 68.8 & 42.8 & 54.6 \\
\hline Second half & 75.6 & 49.8 & 61.4 & 70.2 & 43.3 & 55.3 \\
\hline 1995 & & & & & & \\
\hline First half & 76.3 & 50.9 & 62.3 & 70.1 & 44.1 & 55.8 \\
\hline Second half & 75.3 & 51.4 & 61.9 & 68.8 & 44.3 & 55.1 \\
\hline 1996 & & & & & & \\
\hline First half & 73.7 & 51.2 & 61.3 & 66.1 & 43.5 & 53.6 \\
\hline Second half & 78.3 & 52.9 & 62.0 & 65.5 & 45.5 & 54.5 \\
\hline 1997 & & & & & & \\
\hline First half & 72.2 & 49.4 & 59.6 & 65.1 & 41.9 & 52.4 \\
\hline Second half & 73.1 & 50.6 & 60.7 & 66.7 & 43.5 & 54.0 \\
\hline 1998 & & & & & & \\
\hline First half & 73.3 & 51.3 & 61.1 & 68.1 & 44.6 & 55.1 \\
\hline Second half & 73.8 & 51.8 & 61.8 & 67.2 & 45.3 & 55.2 \\
\hline
\end{tabular}

Source: General Directorate of Statistics.

1/ For Montevideo. 
Table 15. Uruguay: Unemployment Rate $1 /$

(As a percentage of the labor force)

\begin{tabular}{|c|c|c|c|}
\hline & Male & Female & Total \\
\hline \multicolumn{4}{|l|}{1990} \\
\hline First half & 6.7 & 11.7 & 8.9 \\
\hline Second half & 8.0 & 11.9 & 9.7 \\
\hline \multicolumn{4}{|l|}{1991} \\
\hline First half & 7.5 & 12.4 & 9.6 \\
\hline Second half & 6.7 & 10.2 & 8.3 \\
\hline \multicolumn{4}{|l|}{1992} \\
\hline First half & 7.1 & 13.0 & 9.7 \\
\hline Second half & 6.3 & 10.7 & 8.3 \\
\hline \multicolumn{4}{|l|}{1993} \\
\hline First half & 6.4 & 11.9 & 8.9 \\
\hline Second half & 6.3 & 10.0 & 8.0 \\
\hline \multicolumn{4}{|l|}{1994} \\
\hline First half & 6.7 & 10.9 & 8.5 \\
\hline Second half & 7.2 & 13.0 & 9.8 \\
\hline \multicolumn{4}{|l|}{1995} \\
\hline First half & 8.1 & 13.6 & 10.6 \\
\hline Second half & 8.7 & 13.8 & 11.0 \\
\hline \multicolumn{4}{|l|}{1996} \\
\hline First half & 10.3 & 15.2 & 12.6 \\
\hline Second half & 10.7 & 13.9 & 12.2 \\
\hline \multicolumn{4}{|l|}{1997} \\
\hline First half & 9.7 & 15.1 & 12.2 \\
\hline Second half & 8.6 & 14.0 & 11.0 \\
\hline \multicolumn{4}{|l|}{1998} \\
\hline First half & 7.1 & 13.0 & 9.8 \\
\hline Second half & 9.0 & 12.5 & 10.6 \\
\hline
\end{tabular}

Source: General Directorate of Statistics.

1/ For Montevideo. 
Table 16. Uruguay: Price Movements

(Annual percentage change) $1 /$

\begin{tabular}{lrrrrrr}
\hline & 1993 & 1994 & 1995 & 1996 & 1997 & 1998 \\
\hline Consumer prices & & & & & & \\
Food & $\mathbf{5 2 . 9}$ & $\mathbf{4 4 . 1}$ & $\mathbf{3 5 . 4}$ & $\mathbf{2 4 . 3}$ & $\mathbf{1 5 . 2}$ & $\mathbf{8 . 6}$ \\
Housing & 53.4 & 42.9 & 29.5 & 22.2 & 14.0 & 9.2 \\
Clothing & 62.1 & 50.0 & 41.6 & 28.1 & 19.5 & 8.9 \\
& 35.9 & 30.8 & 33.7 & 16.1 & 7.1 & 8.0 \\
Wholesale prices & & & & & & \\
Manufactures & $\mathbf{3 1 . 1}$ & $\mathbf{4 1 . 0}$ & $\mathbf{2 7 . 7}$ & $\mathbf{2 3 . 5}$ & $\mathbf{1 3 . 3}$ & $\mathbf{3 . 3}$ \\
Agricultural products & 34.7 & 34.9 & 31.3 & 21.6 & 13.0 & 5.4 \\
& 21.4 & 60.1 & 18.4 & 28.8 & 13.7 & -2.9 \\
\hline
\end{tabular}

Source: General Directorate of Statistics.

1/ End of period. 
Table 17. Uruguay: Public Utility and Petroleum Prices

\begin{tabular}{|c|c|c|c|c|c|c|c|c|}
\hline & \multicolumn{2}{|c|}{ Electricity } & \multirow[b]{2}{*}{ Water } & \multirow{2}{*}{$\begin{array}{r}\text { Natural } \\
\text { Gas }\end{array}$} & \multirow{2}{*}{$\begin{array}{r}\text { Montevideo } \\
\text { Transport }\end{array}$} & \multirow{2}{*}{$\begin{array}{r}\text { Regular } \\
\text { Gasoline }\end{array}$} & \multirow{2}{*}{$\begin{array}{r}\text { Gas } \\
\text { Oil }\end{array}$} & \multirow{2}{*}{$\begin{array}{r}\text { Fuel } \\
\text { Oil }\end{array}$} \\
\hline & Household & Industrial & & & & & & \\
\hline \multicolumn{9}{|c|}{ I. Percentage Change During Period } \\
\hline 1992 & 51.0 & 50.0 & 64.0 & 37.0 & 56.0 & 36.0 & 15.0 & 23.0 \\
\hline 1993 & 61.0 & 67.0 & 77.0 & 44.0 & 43.0 & 38.0 & 27.0 & 4.0 \\
\hline 1994 & 64.0 & 55.0 & 57.0 & 35.0 & 58.0 & 38.0 & 38.0 & 8.0 \\
\hline 1995 & 52.0 & 47.0 & 46.0 & 39.0 & 59.0 & 34.0 & 37.0 & 22.0 \\
\hline 1996 & 31.3 & 32.0 & 34.9 & 25.8 & 42.0 & 32.3 & 40.4 & 28.0 \\
\hline 1997 & 22.1 & 23.0 & 29.3 & 22.6 & 25.2 & 24.2 & 24.0 & 22.6 \\
\hline 1998 & 10.9 & 11.5 & 11.2 & 10.4 & 11.8 & 5.2 & 4.8 & 4.8 \\
\hline \multicolumn{9}{|l|}{1996} \\
\hline January & 0.0 & 0.0 & 11.3 & 6.2 & 0.0 & 0.0 & 0.0 & 0.0 \\
\hline February & 4.6 & 4.3 & 0.0 & 0.0 & 0.0 & 3.6 & 8.6 & 3.3 \\
\hline March & 0.0 & 0.0 & 0.0 & 0.0 & 0.0 & 0.0 & 0.0 & 0.0 \\
\hline April & 3.4 & 3.4 & 0.0 & 4.7 & 11.6 & 8.8 & 12.6 & 8.9 \\
\hline May & 0.0 & 0.0 & 11.9 & 0.0 & 0.0 & 0.0 & 0.0 & 0.0 \\
\hline June & 4.8 & 5.1 & 0.0 & 0.0 & 0.0 & 4.8 & 3.8 & 4.0 \\
\hline July & 0.0 & 0.0 & 0.0 & 8.8 & 5.6 & 0.0 & 0.0 & 0.0 \\
\hline August & 3.7 & 2.7 & 0.0 & 0.0 & 0.9 & 4.6 & 4.5 & 4.5 \\
\hline September & 0.0 & 0.0 & 6.1 & 0.0 & 5.0 & 0.0 & 0.0 & 0.0 \\
\hline October & 4.0 & 4.4 & 0.0 & 4.6 & 4.8 & 5.9 & 5.1 & 5.0 \\
\hline Novembar & 0.0 & 0.0 & 0.0 & 0.0 & 0.0 & 0.0 & 0.0 & 0.0 \\
\hline December & 3.8 & 3.8 & 0.0 & 0.0 & 0.0 & 8.3 & 8.2 & 8.4 \\
\hline \multicolumn{9}{|l|}{1997} \\
\hline January & 0.0 & 0.0 & 14.3 & 8.1 & 6.1 & 0.0 & 0.0 & 0.0 \\
\hline February & 3.2 & 3.9 & 0.0 & 0.0 & 0.4 & 0.0 & 0.0 & 0.0 \\
\hline March & 0.0 & 0.0 & 0.0 & 0.0 & 0.0 & 0.0 & 0.0 & 0.0 \\
\hline April & 3.1 & 3.2 & 0.0 & 2.6 & 0.0 & 0.0 & 0.0 & 0.0 \\
\hline May & 0.0 & 0.0 & 5.5 & 0.0 & 0.0 & 0.0 & 0.0 & 0.0 \\
\hline June & 2.6 & 2.9 & 0.0 & 0.0 & 0.0 & 5.1 & 4.8 & 4.5 \\
\hline July & 0.0 & 0.0 & 0.0 & 3.6 & 5.4 & 0.0 & 0.0 & 0.0 \\
\hline August & 3.1 & 2.9 & 0.0 & 0.0 & 3.0 & 0.0 & 0.0 & 0.0 \\
\hline September & 0.0 & 0.0 & 4.0 & 0.0 & 0.0 & 0.0 & 0.0 & 0.0 \\
\hline October & 1.7 & 1.9 & 0.0 & 0.0 & 0.0 & 0.0 & 0.0 & 0.0 \\
\hline November & 0.0 & 0.0 & 0.0 & 2.1 & 0.0 & 3.7 & 3.4 & 3.5 \\
\hline December & 1.7 & 1.7 & 0.0 & 0.0 & 0.0 & 0.0 & 0.0 & 0.0 \\
\hline \multicolumn{9}{|l|}{1998} \\
\hline January & 0.0 & 0.0 & 4.2 & 2.9 & 5.6 & 0.0 & 0.0 & 0.0 \\
\hline February & 3.1 & 3.0 & 0.1 & 0.0 & 1.6 & 0.0 & 0.0 & 0.0 \\
\hline March & 0.0 & 0.0 & 0.0 & 0.0 & 0.0 & 0.0 & 0.0 & 0.0 \\
\hline April & 0.0 & 0.0 & 0.0 & 2.2 & 0.0 & 0.0 & 0.0 & 0.0 \\
\hline May & 0.0 & 0.0 & 0.0 & 0.0 & 0.0 & 0.0 & 0.0 & 0.0 \\
\hline June & 0.0 & 0.0 & 0.0 & 0.0 & 0.0 & 0.0 & 0.0 & 0.0 \\
\hline July & 0.0 & 0.0 & 4.3 & 1.2 & 0.0 & 0.0 & 0.0 & 0.0 \\
\hline August & 1.8 & 3.2 & 0.0 & 0.0 & 0.0 & 0.0 & 0.0 & 0.0 \\
\hline September & 0.0 & 0.0 & 0.0 & 0.0 & 0.0 & 0.0 & 0.0 & 0.0 \\
\hline October & 0.0 & 0.0 & 0.0 & 2.1 & 0.0 & 0.0 & 0.0 & 0.0 \\
\hline November & 0.0 & 0.0 & 0.0 & 0.0 & 0.0 & 0.0 & 0.0 & 0.0 \\
\hline December & 0.0 & 0.0 & 0.0 & 0.0 & 0.0 & 0.0 & 0.0 & 0.0 \\
\hline
\end{tabular}


Table 17. Uruguay: Public Utility and Petroleum Prices

\begin{tabular}{|c|c|c|c|c|c|c|c|c|}
\hline & \multicolumn{2}{|c|}{ Electricity } & \multirow[b]{2}{*}{ Water } & \multirow{2}{*}{$\begin{array}{r}\text { Natural } \\
\text { Gas }\end{array}$} & \multirow{2}{*}{$\begin{array}{r}\text { Montevideo } \\
\text { Transport }\end{array}$} & \multirow{2}{*}{$\begin{array}{l}\text { Regular } \\
\text { Gasoline }\end{array}$} & \multirow{2}{*}{$\begin{array}{r}\text { Gas } \\
\text { Oil }\end{array}$} & \multirow{2}{*}{$\begin{array}{r}\text { Fuel } \\
\text { Oil }\end{array}$} \\
\hline & Household & Industrial & & & & & & \\
\hline \multicolumn{9}{|c|}{ II. End of Period Index Deflated by Cost of Living $(1983=100)$} \\
\hline 1990 & 93.7 & 93.7 & 53.0 & 137.2 & 80.8 & 169.7 & 200.4 & 286.5 \\
\hline 1991 & 85.2 & 85.3 & 64.0 & 127.7 & 84.4 & 134.0 & 157.5 & 257.5 \\
\hline 1992 & 81.0 & 80.6 & 66.0 & 110.2 & 82.9 & 130.9 & 114.0 & 200.0 \\
\hline 1993 & 87.6 & 91.7 & 81.9 & 100.4 & 74.7 & 111.4 & 84.5 & 102.2 \\
\hline \multicolumn{9}{|l|}{1994} \\
\hline March & 87.4 & 90.7 & 85.0 & 102.5 & 77.9 & 109.7 & 87.7 & 126.4 \\
\hline June & 91.0 & 95.5 & 86.2 & 102.5 & 77.5 & 119.6 & 95.5 & 115.5 \\
\hline September & 96.1 & 92.3 & 92.5 & 108.2 & 77.3 & 116.1 & 93.3 & 104.9 \\
\hline December & 96.6 & 94.9 & 83.2 & 97.5 & 85.0 & 113.0 & 90.9 & 102.0 \\
\hline \multicolumn{9}{|l|}{1995} \\
\hline March & 94.6 & 92.7 & 85.3 & 99.9 & 78.2 & 103.9 & 90.5 & 101.7 \\
\hline June & 98.5 & 99.1 & 96.8 & 98.1 & 84.1 & 109.5 & 89.4 & 101.7 \\
\hline September & 96.7 & 97.6 & 91.3 & 96.4 & 89.6 & 105.4 & 87.5 & 94.5 \\
\hline December & 99.7 & 100.4 & 86.4 & 95.4 & 93.6 & 105.6 & 86.4 & 93.7 \\
\hline \multicolumn{9}{|l|}{1996} \\
\hline March & 98.7 & 99.2 & 91.2 & 95.9 & 88.7 & 90.3 & 88.9 & 91.8 \\
\hline June & 100.6 & 101.4 & 95.9 & 94.5 & 93.1 & 96.8 & 97.7 & 97.8 \\
\hline September & 98.1 & 97.8 & 95.6 & 96.6 & 97.9 & 95.1 & 95.9 & 96.8 \\
\hline December & 101.8 & 101.9 & 91.8 & 97.1 & 98.5 & 104.9 & 104.9 & 105.0 \\
\hline \multicolumn{9}{|l|}{1997} \\
\hline March & 100.0 & 100.0 & 100.0 & 100.0 & 100.0 & 100.0 & 100.0 & 100.0 \\
\hline June & 101.6 & 102.0 & 101.3 & 98.5 & 96.0 & 108.8 & 98.5 & 100.3 \\
\hline September & 101.8 & 102.0 & 102.4 & 99.3 & 101.3 & 97.9 & 99.8 & 97.4 \\
\hline December & 102.8 & 103.3 & 100.0 & 99.0 & 99.0 & 99.2 & 98.7 & 98.6 \\
\hline \multicolumn{9}{|l|}{1998 (Prel.) } \\
\hline March & 103.4 & 103.9 & 101.8 & 99.5 & 103.8 & 97.0 & 96.4 & 96.3 \\
\hline June & 101.3 & 101.7 & 99.7 & 99.6 & 101.6 & 95.0 & 94.4 & 94.3 \\
\hline September & 100.3 & 102.2 & 101.2 & 98.2 & 98.9 & 92.4 & 91.9 & 91.7 \\
\hline December & 99.2 & 101.0 & 100.1 & 99.1 & 97.8 & 91.4 & 90.9 & 90.7 \\
\hline
\end{tabular}

Source: Central Bank of Uruguay. 
Table 18. Uruguay: Wage Developments

(Percentage change over the corresponding period of the previous year; period average)

\begin{tabular}{|c|c|c|c|c|c|c|c|}
\hline & \multicolumn{2}{|c|}{ Public Sector } & \multicolumn{3}{|c|}{ Private Sector } & \multicolumn{2}{|c|}{ Overall Wage Index } \\
\hline & Nominal & Real 1/ & Nominal & Real 1/ & In US\$ & Nominal & Real 1/ \\
\hline 1993 & 66.2 & 7.4 & 59.0 & 3.3 & 22.1 & 61.7 & 4.8 \\
\hline 1994 & 45.6 & 0.6 & 46.3 & 1.2 & 14.6 & 46.1 & 1.0 \\
\hline 1995 & 38.6 & -2.6 & 37.8 & -3.1 & 9.6 & 38.1 & -2.9 \\
\hline 1996 & 32.0 & 2.9 & 28.5 & 0.1 & 2.4 & 29.3 & 0.7 \\
\hline 1997 & 21.3 & 1.3 & 19.6 & -0.2 & 1.0 & 20.1 & 0.3 \\
\hline 1998 & 14.3 & 3.1 & 12.1 & 1.2 & 2.3 & 12.8 & 1.8 \\
\hline \multicolumn{8}{|l|}{1994} \\
\hline 1st quarter & 49.7 & 0.1 & 50.7 & 0.8 & 18.9 & 50.3 & 0.5 \\
\hline 2nd quarter & 45.6 & 0.7 & 49.3 & 3.3 & 18.1 & 47.9 & 2.3 \\
\hline 3rd quarter & 45.1 & 2.3 & 45.1 & 2.2 & 12.4 & 45.1 & 2.3 \\
\hline 4th quarter & 43.0 & -0.7 & 41.3 & -1.9 & 9.4 & 41.9 & -1.4 \\
\hline \multicolumn{8}{|l|}{1995} \\
\hline 1st quarter & 43.5 & -0.7 & 43.8 & -0.5 & 12.4 & 43.7 & -0.6 \\
\hline 2nd quarter & 39.3 & -3.9 & 39.3 & -3.9 & 9.6 & 39.3 & -3.9 \\
\hline 3rd quarter & 36.6 & -4.8 & 35.8 & -5.3 & 9.5 & 36.1 & -5.2 \\
\hline 4th quarter & 35.9 & -0.9 & 34.0 & -2.4 & 7.2 & 34.7 & -1.8 \\
\hline \multicolumn{8}{|l|}{1996} \\
\hline 1st quarter & 32.8 & -0.1 & 29.2 & -2.8 & 2.6 & 30.0 & -2.2 \\
\hline 2nd quarter & 33.7 & 3.1 & 30.5 & 0.6 & 3.3 & 31.1 & 1.1 \\
\hline 3rd quarter & 33.2 & 5.4 & 29.3 & 2.3 & 2.1 & 30.2 & 3.0 \\
\hline 4th quarter & 28.9 & 2.8 & 25.5 & 0.1 & 1.7 & 26.4 & 0.8 \\
\hline \multicolumn{8}{|l|}{1997} \\
\hline 1st quarter & 25.0 & 0.7 & 23.5 & -0.5 & 2.1 & 24.0 & -0.1 \\
\hline 2nd quarter & 22.5 & 0.9 & 20.6 & -0.7 & 0.8 & 21.2 & -0.2 \\
\hline 3rd quarter & 19.7 & 1.7 & 18.6 & -0.3 & 1.2 & 18.9 & 0.0 \\
\hline 4th quarter & 18.8 & 2.7 & 16.4 & 0.6 & 0.4 & 17.1 & 1.3 \\
\hline \multicolumn{8}{|l|}{1998} \\
\hline 1st quarter & 17.7 & 3.8 & 14.9 & 1.3 & 2.2 & 15.8 & 2.1 \\
\hline 2nd quarter & 13.8 & 2.7 & 13.0 & 2.1 & 2.8 & 13.3 & 2.3 \\
\hline 3rd quarter & 14.4 & 3.9 & 11.1 & 0.9 & 2.1 & 12.1 & 1.8 \\
\hline 4th quarter & 11.5 & 2.1 & 9.9 & 0.5 & 2.2 & 10.4 & 1.0 \\
\hline
\end{tabular}

Sources: Central Bank of Uruguay; and Fund staff estimates.

1/ Deflated by the consumer price index. 
Table 19. Uruguay: Public Sector Operations

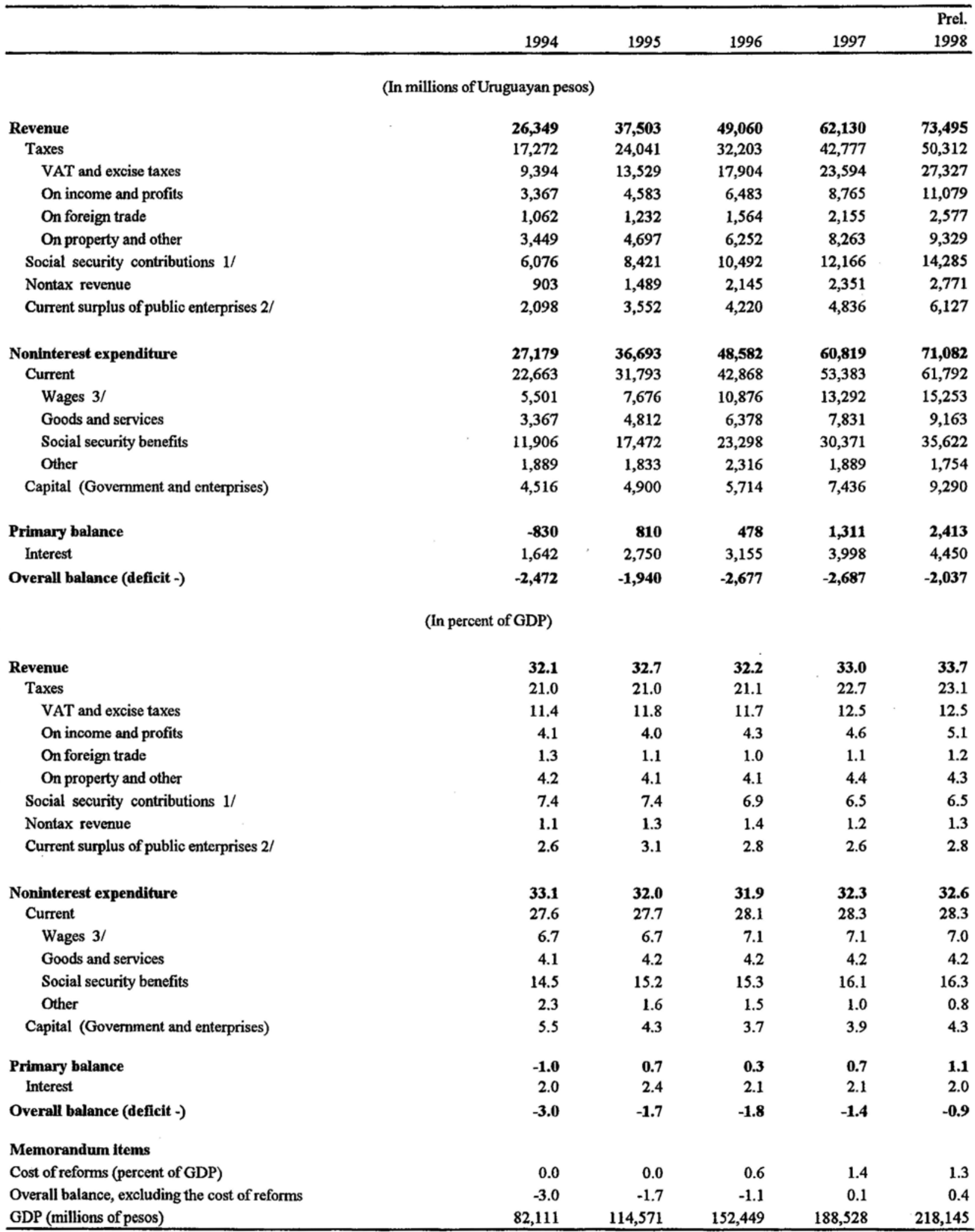

Sources: Ministry of Finance; and Fund staff estimates.

1/ Excludes contributions that are transferred to the private pension funds.

2/ Before interest expenditures.

3/ Includes severance payments under the Reform of the State.

CInternational Monetary Fund. Not for Redistribution 
Table 20. Uruguay: Summary Operations of the Central Government

\begin{tabular}{|c|c|c|c|c|c|}
\hline & 1994 & 1995 & 1996 & 1997 & $\begin{array}{r}\text { Prel. } \\
1998 \\
\end{array}$ \\
\hline \multicolumn{6}{|c|}{ (In millions of Uruguayan pesos) } \\
\hline Total revenue & 15,355 & 21,818 & 29,189 & $\mathbf{3 8 , 5 4 2}$ & 45,721 \\
\hline Import duties & 1,067 & 1,260 & 1,564 & 2,155 & 2,577 \\
\hline Domestic taxes & 12,481 & 18,217 & 24,518 & 31,843 & 37,725 \\
\hline Of which: collections from tax authority & 11,906 & 16,727 & 22,734 & 29,889 & 35,195 \\
\hline Negotiated transfers from public enterprises 1/ & 575 & 573 & 794 & 1,593 & 1,994 \\
\hline Other nontax revenue & 1,232 & 1,768 & 2,313 & 2,951 & 3,425 \\
\hline Total noninterest expenditures & 16,012 & 21,998 & $\mathbf{3 0 , 1 1 7}$ & 38,874 & 45,381 \\
\hline Wages & 4,352 & 5,499 & 7,627 & 9,413 & 10,764 \\
\hline Goods and services & 2,381 & 3,437 & 4,142 & 5,234 & 6,274 \\
\hline Social security expenditures $2 /$ & 6,240 & 9,051 & 13,656 & 17,895 & 20,659 \\
\hline Transfers to social security administration & 4,845 & 7,103 & 11,072 & 14,621 & 16,810 \\
\hline Police pensions & 493 & 687 & 900 & 1,172 & 1,331 \\
\hline Military pensions & 903 & 1,260 & 1,684 & 2,102 & 2,518 \\
\hline Other transfers and subsidies & 821 & 1,375 & 1,879 & 2,567 & 2,481 \\
\hline Capital expenditures & 2,217 & 2,635 & 2,813 & 3,765 & 5,203 \\
\hline Primary balance & -657 & -179 & -928 & -332 & 340 \\
\hline Interest payments & 1,067 & 1,719 & 2,047 & 2,845 & 3,092 \\
\hline Overall balance & $-1,724$ & $-1,898$ & $-2,975$ & $-3,177$ & $-2,752$ \\
\hline \multicolumn{6}{|c|}{ (In percent of GDP) } \\
\hline Total revenue & 18.7 & 19.0 & 19.1 & 20.4 & $\mathbf{2 1 . 0}$ \\
\hline Import duties & 1.3 & 1.1 & 1.0 & 1.1 & 1.2 \\
\hline Domestic taxes & 15.2 & 15.9 & 16.1 & 16.9 & 17.3 \\
\hline Of which: collections from tax authority & 14.5 & 14.6 & 14.9 & 15.9 & 16.1 \\
\hline Negotiated transfers from public enterprises $1 /$ & 0.7 & 0.5 & 0.4 & 0.8 & 0.9 \\
\hline Other nontax revenue & 1.5 & 1.5 & 1.5 & 1.5 & 1.5 \\
\hline Total noninterest expenditures & 19.5 & 19.2 & 19.8 & 20.6 & 20.8 \\
\hline Wages & 5.3 & 4.8 & 5.0 & 5.0 & 4.9 \\
\hline Goods and services & 2.9 & 3.0 & 2.7 & 2.8 & 2.9 \\
\hline Social security expenditures $2 /$ & 7.6 & 7.9 & 9.0 & 9.5 & 9.5 \\
\hline Transfers to social security administration & 5.9 & 6.2 & 7.3 & 7.8 & 7.7 \\
\hline Police pensions & 0.6 & 0.6 & 0.6 & 0.6 & 0.6 \\
\hline Military pensions & 1.1 & 1.1 & 1.1 & 1.1 & 1.2 \\
\hline Other transfers and subsidies & 1.0 & 1.2 & 1.2 & 1.4 & 1.1 \\
\hline Capital expenditures & 2.7 & 2.3 & 1.8 & 2.0 & 2.4 \\
\hline Primary balance & -0.8 & -0.2 & -0.6 & -0.2 & 0.2 \\
\hline Interest payments & 1.3 & 1.5 & 1.3 & 1.5 & 1.4 \\
\hline Overall balance & -2.1 & -1.7 & -2.0 & -1.7 & -1.2 \\
\hline \multicolumn{6}{|l|}{ Memorandum items } \\
\hline Cost of reforms & 0.0 & 0.0 & 0.4 & 1.1 & 1.2 \\
\hline Central administration reform & 0.0 & 0.0 & 0.1 & 0.3 & 0.3 \\
\hline Contribution to AFAPs & 0.0 & 0.0 & 0.4 & 0.8 & 0.9 \\
\hline
\end{tabular}

Sources: Ministry of Finance; and Fund staff estimates.

1/ In addition to statutory tax obligations.

2/ Includes employer contribution for central administration employees. 
Table 21. Uruguay: Summary Operations of the Social Security System

(In millions of Uruguayan pesos)

\section{Revenue}

Contributions and taxes

Transfers from the central government

\section{Expenditure \\ Wages \\ Goods and services \\ Capital spending \\ Benefits \\ Pensions}

Other social security benefits

Transfers $1 /$

Balance (deficit -)

$\begin{array}{rrrrr}\mathbf{1 2 , 5 3 9} & \mathbf{1 7 , 7 6 1} & \mathbf{2 3 , 8 6 7} & \mathbf{3 0 , 4 9 1} & \mathbf{3 5 , 6 8 5} \\ \mathbf{7 , 6 7 7} & 10,643 & 12,854 & \mathbf{1 5 , 9 6 2} & \mathbf{1 8 , 9 3 2} \\ 4,862 & 7,117 & 11,013 & 14,529 & \mathbf{1 6 , 7 5 3} \\ \mathbf{1 2 , 4 8 6} & \mathbf{1 7 , 7 6 0} & \mathbf{2 3 , 8 1 2} & \mathbf{3 0 , 5 4 8} & \mathbf{3 5 , 7 4 8} \\ 370 & \mathbf{5 7 3} & \mathbf{7 7 5} & \mathbf{7 5 8} & \mathbf{8 5 0} \\ 328 & 458 & \mathbf{5 5 4} & \mathbf{5 0 1} & \mathbf{5 0 3} \\ \ldots & \ldots & \ldots & 50 & 128 \\ 10,803 & 15,355 & 19,952 & 25,212 & 29,235 \\ \mathbf{8 , 7 0 6} & 12,374 & 16,079 & 20,285 & 23,369 \\ 2,097 & 2,981 & 3,873 & 4,927 & \mathbf{5 , 8 6 6} \\ \mathbf{9 8 5} & \mathbf{1 , 3 7 5} & 2,531 & 4,027 & \mathbf{5 , 0 3 3} \\ & & & & \\ \mathbf{5 3} & \mathbf{0} & \mathbf{5 5} & \mathbf{- 5 7} & \mathbf{6 3}\end{array}$

(In percent of GDP)

\section{Revenue}

Contributions and taxes

Transfers from the central government

\section{Expenditure}

Wages

Goods and services

Capital spending

Benefits

Pensions

Other social security benefits

Transfers 1/

$\begin{array}{rrrrr}\mathbf{1 5 . 3} & \mathbf{1 5 . 5} & \mathbf{1 5 . 7} & \mathbf{1 6 . 2} & \mathbf{1 6 . 4} \\ 9.3 & 9.3 & 8.4 & 8.5 & \mathbf{8 . 7} \\ 5.9 & 6.2 & 7.2 & 7.7 & 7.7 \\ \mathbf{1 5 . 2} & \mathbf{1 5 . 5} & \mathbf{1 5 . 6} & \mathbf{1 6 . 2} & \mathbf{1 6 . 4} \\ 0.5 & 0.5 & 0.5 & 0.4 & 0.4 \\ 0.4 & 0.4 & 0.4 & 0.3 & 0.2 \\ \ldots & \ldots & \ldots & 0.0 & 0.1 \\ 13.2 & 13.4 & 13.1 & 13.4 & 13.4 \\ 10.6 & 10.8 & 10.5 & 10.8 & 10.7 \\ 2.6 & 2.6 & 2.5 & 2.6 & 2.7 \\ 1.2 & 1.2 & 1.7 & 2.1 & 2.3\end{array}$

\section{Balance (deficit -)}

$0.1 \quad 0.0$

0.0

0.0

0.0

Sources: Social Security Administration (BPS) and Ministry of Economy.

1/ Includes transfers to the Treasury of taxes collected by the social security administration and transfers to the private pension funds. 
Table 22. Uruguay: Local Government Finances

\begin{tabular}{|c|c|c|c|c|c|}
\hline & 1994 & 1995 & 1996 & 1997 & $\begin{array}{r}\text { Est. } \\
1998 \\
\end{array}$ \\
\hline \multicolumn{6}{|c|}{ (In millions of Uruguayan pesos) } \\
\hline Revenue & 2,792 & 3,895 & 5,159 & 6,586 & 7,726 \\
\hline Taxes & 2,710 & 3,781 & 5,006 & 6,396 & 7,663 \\
\hline Other & 82 & 115 & 153 & 190 & 63 \\
\hline Expenditure & 2,842 & 3,857 & 5,101 & 6,308 & 7,313 \\
\hline Wages & 1,150 & 1,604 & 2,177 & 2,693 & 3,135 \\
\hline Goods and services & 903 & 1,260 & 1,608 & 1,989 & 2,260 \\
\hline Social security expenses & 246 & 344 & 491 & 608 & 690 \\
\hline Other & 234 & 328 & 479 & 593 & 679 \\
\hline Capital & 309 & 321 & 345 & 426 & 549 \\
\hline Primary balance (deficit -) & -50 & 39 & 58 & 278 & 413 \\
\hline Interest & 24 & 33 & 44 & 54 & 63 \\
\hline Overall balance (deficit -) & -74 & 6 & 14 & 224 & 350 \\
\hline \multicolumn{6}{|c|}{ (In percent of GDP) } \\
\hline Revenue & 3.4 & 3.4 & 3.4 & 3.5 & 3.5 \\
\hline Taxes & 3.3 & 3.3 & 3.3 & 3.4 & 3.5 \\
\hline Other & 0.1 & 0.1 & 0.1 & 0.1 & 0.0 \\
\hline Expenditure & 3.5 & 3.4 & 3.3 & 3.3 & 3.3 \\
\hline Wages & 1.4 & 1.4 & 1.4 & 1.4 & 1.4 \\
\hline Goods and services & 1.1 & 1.1 & 1.1 & 1.1 & 1.0 \\
\hline Social security expenses & 0.3 & 0.3 & 0.3 & 0.3 & 0.3 \\
\hline Other & 0.3 & 0.3 & 0.3 & 0.3 & 0.3 \\
\hline Capital & 0.4 & 0.3 & 0.2 & 0.2 & 0.3 \\
\hline Primary balance (deficit -) & -0.1 & 0.0 & 0.0 & 0.1 & 0.2 \\
\hline Interest & 0.0 & 0.0 & 0.0 & 0.0 & 0.0 \\
\hline Overall balance (deficit -) & -0.1 & 0.0 & 0.0 & 0.1 & 0.2 \\
\hline
\end{tabular}

Sources: Ministry of Economy. 
Table 23. Uruguay: Consolidated Public Sector Enterprises and Autonomous Agencies

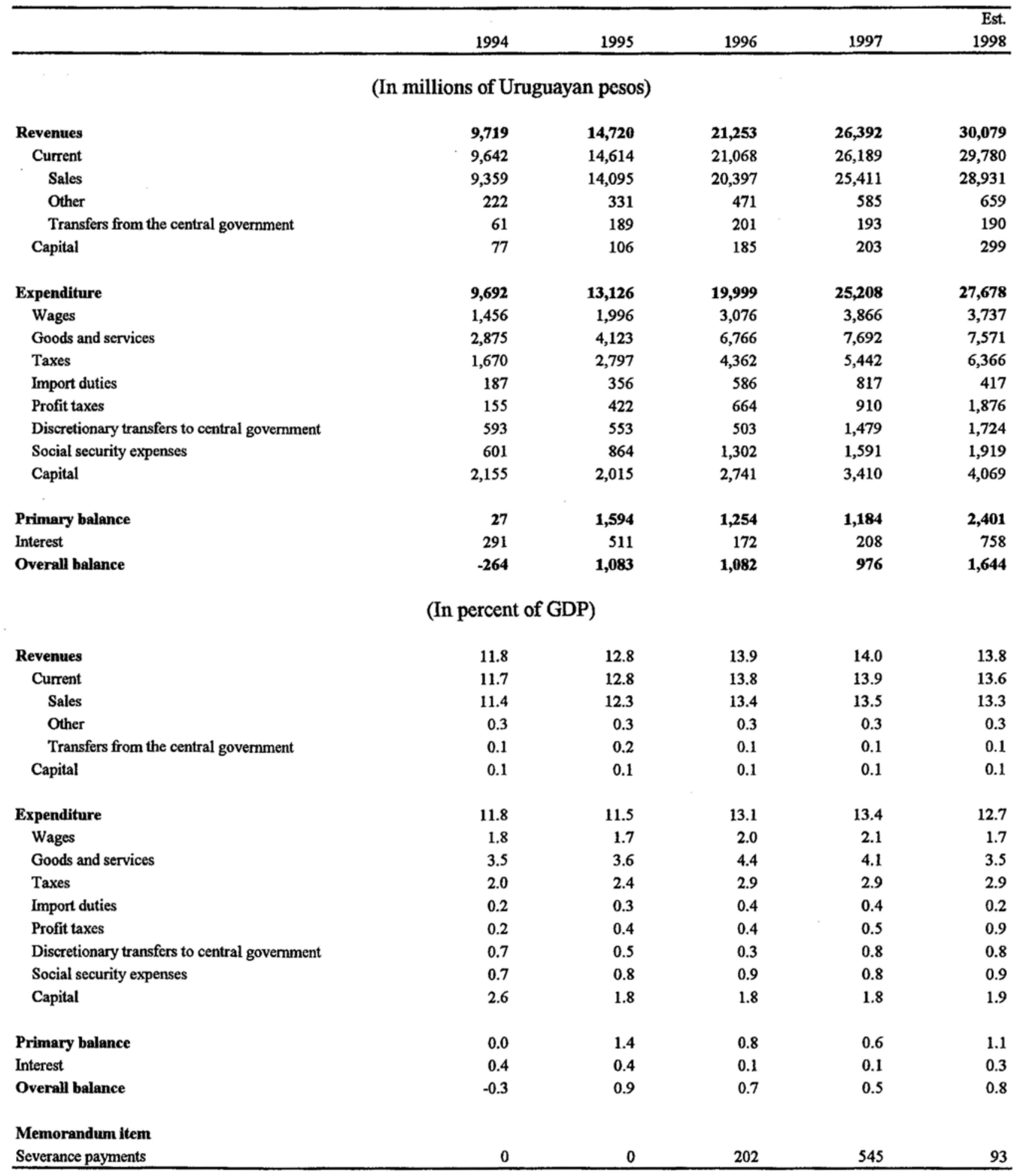

Sources: Ministry of Economy and Office of Budget and Planning. 
Table 24. Uruguay: Quasi Fiscal Result of the Central Bank

1994

1995

1996

1997

1998

(In millions of Uruguayan pesos)

\section{Revenue}

Interest on international reserves

Receipts on other foreign currency assets

Receipts on peso assets

Expenditure

Operating costs

Wages

Goods and services

Interest on foreign debt

Interest on peso debt

Other expenses on foreign currency liabilities

Other expenses in pesos

Overall balance (deficit -)

$\begin{array}{rrrrr}\mathbf{5 9 4} & \mathbf{9 4 8} & \mathbf{1 , 2 8 2} & \mathbf{1 , 5 8 6} & \mathbf{1 , 6 4 6} \\ 187 & 392 & 577 & 668 & 851 \\ 372 & 516 & 698 & 882 & 782 \\ 35 & 40 & 7 & 36 & 13\end{array}$

$1,113 \quad 1,623$

2,129

371

2,539

537

2,732

$139 \quad 276$

211

297

430

630

106

65

74

107

504

$502 \quad 700$

1,359

1,412

126

$441 \quad 553$

329

401

1,528

$23 \quad 78$

53

165

327

233

17

24

14

$-519$

$-675$

$-847$

$-953$

$-1,086$

(In percent of GDP)

\begin{tabular}{llllll} 
Revenue & 0.7 & 0.8 & 0.8 & 0.8 & 0.8 \\
Interest on international reserves & 0.2 & 0.3 & 0.4 & 0.4 & 0.4 \\
Receipts on other foreign currency assets & 0.5 & 0.5 & 0.5 & 0.5 & 0.4 \\
Receipts on peso assets & 0.0 & 0.0 & 0.0 & 0.0 & 0.0 \\
& & & & & \\
Expenditure & 1.4 & 1.4 & 1.4 & 1.3 & 1.3 \\
Operating costs & 0.2 & 0.2 & 0.2 & 0.3 & 0.3 \\
$\quad$ Wages & 0.1 & 0.2 & 0.2 & 0.2 & 0.2 \\
$\quad$ Goods and services & 0.0 & 0.1 & 0.0 & 0.1 & 0.1 \\
Interest on foreign debt & 0.6 & 0.6 & 0.9 & 0.7 & 0.7 \\
Interest on peso debt & 0.5 & 0.5 & 0.2 & 0.2 & 0.1 \\
Other expenses on foreign currency liabilities & 0.0 & 0.1 & 0.0 & 0.1 & 0.1 \\
Other expenses in pesos & 0.0 & 0.0 & 0.0 & 0.0 & 0.0 \\
& & & & & \\
Overall balance (deficit -) & -0.6 & -0.6 & -0.6 & -0.5 & -0.5 \\
\hline
\end{tabular}

Source: Central Bank of Uruguay. 
Table 25. Uruguay: Collections of the Central Tax Authority

(In millions of Uruguayan pesos)

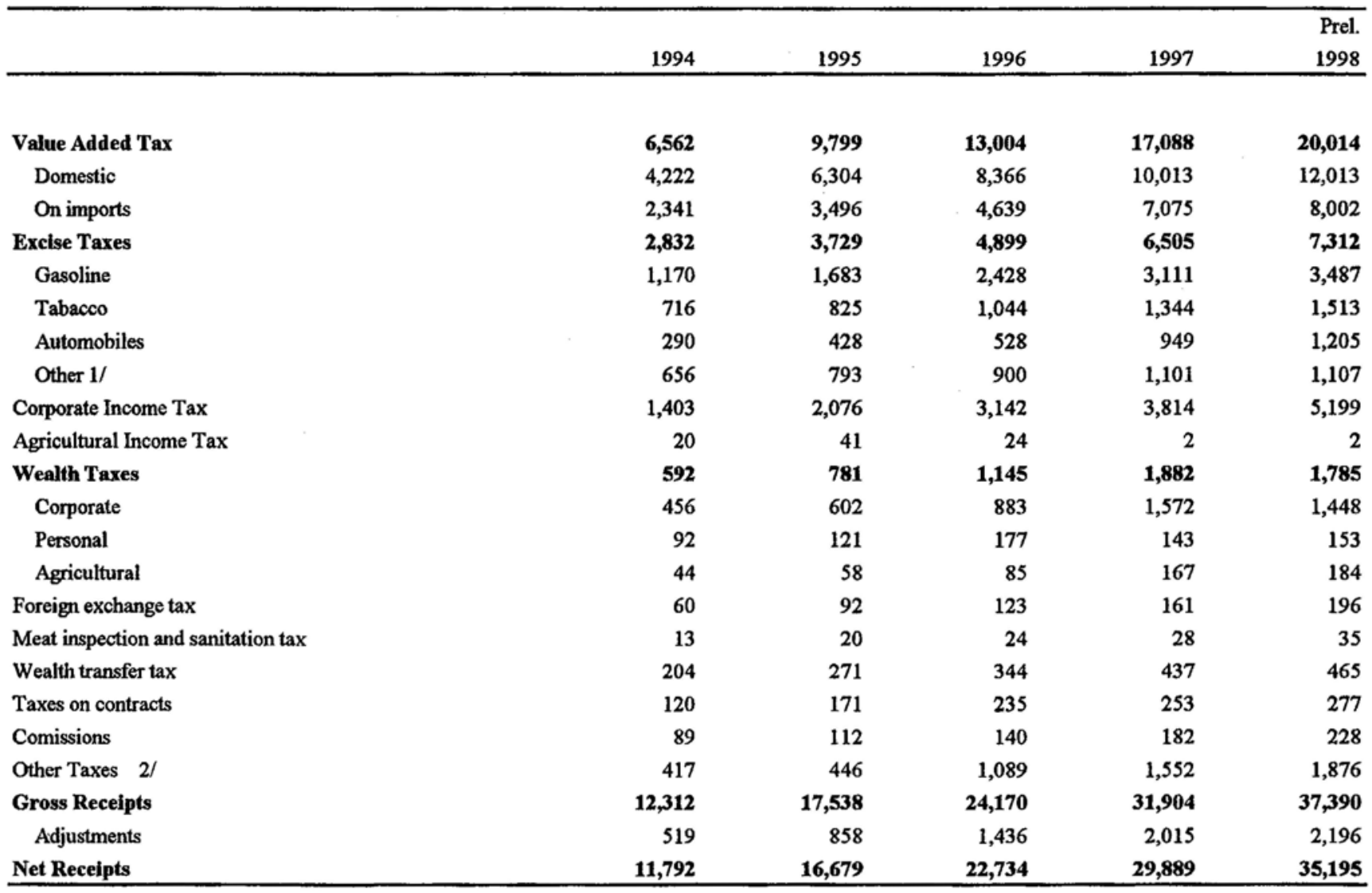

Source: Ministry of Economy and Finance.

1/ Includes Import Commitments

2/ Includes Taxes on Agricultural Sales and Bank Assets. 
Table 26. Uruguay: Operations of the Public Enterprises and Autonomous Agencies

(In millions of Uruguayan pesos)

\begin{tabular}{|c|c|c|c|c|c|}
\hline & 1994 & 1995 & 1996 & 1997 & $\begin{array}{r}\text { Est. } \\
1998 \\
\end{array}$ \\
\hline \multicolumn{6}{|c|}{ I. State Telephone Company (ANTEL) } \\
\hline Revenues & 2,103 & 3,251 & 4,977 & 6,396 & $\mathbf{7 , 8 5 4}$ \\
\hline Current & 2,103 & 3,251 & 4,977 & 6,396 & 7,854 \\
\hline Sales & 2,060 & 3,184 & 4,874 & 6,264 & 7,691 \\
\hline Other & 43 & 67 & 103 & 132 & 162 \\
\hline Transfers from the central government & 0 & 0 & 0 & 0 & 0 \\
\hline Capital & 0 & 0 & 0 & 0 & 0 \\
\hline Expenditure & 2,007 & 2,782 & 4,353 & 6,066 & 7,337 \\
\hline Wages & 307 & 433 & 642 & 765 & 928 \\
\hline Goods and services & 356 & 499 & 826 & 1,253 & 1,750 \\
\hline Taxes & 231 & 385 & 639 & 911 & 1,111 \\
\hline Import duties & 147 & 245 & 405 & 579 & 109 \\
\hline Profit taxes & 14 & 24 & 40 & 57 & 775 \\
\hline Discretionary transfers to central government & 129 & 259 & 324 & 761 & 1,056 \\
\hline Social security expenses & 138 & 196 & 296 & 435 & 550 \\
\hline Capital & 686 & 741 & 1,181 & 1,305 & 1,057 \\
\hline Primary balance & 96 & 469 & 624 & 330 & $\mathbf{5 1 7}$ \\
\hline Interest & 37 & 33 & 43 & 61 & 66 \\
\hline Overall balance & 59 & 436 & $\mathbf{5 8 0}$ & 270 & 451 \\
\hline \multicolumn{6}{|c|}{ II. State Power Company (UTE) } \\
\hline Revenues & 2,794 & 4,478 & 6,501 & 7,902 & 9,089 \\
\hline Current & 2,735 & 4,372 & 6,328 & 7,715 & 8,865 \\
\hline Sales & 2,679 & 4,283 & 6,199 & 7,557 & 8,683 \\
\hline Other & 56 & 89 & 129 & 158 & 181 \\
\hline Transfers from the central government & 0 & 0 & 0 & 0 & 0 \\
\hline Capital & 59 & 106 & 173 & 187 & 225 \\
\hline Expenditure & 2,599 & 3,492 & 5,665 & 7,445 & 7,623 \\
\hline Wages & 494 & 678 & 1,026 & 1,478 & 1,172 \\
\hline Goods and services & 659 & 885 & 1,823 & 2,045 & 1,483 \\
\hline Taxes & 72 & 405 & 750 & 948 & 1,322 \\
\hline Import duties & 11 & 64 & 119 & 151 & 210 \\
\hline Profit taxes & 40 & 226 & 419 & 529 & 738 \\
\hline Discretionary transfers to central government & 303 & 101 & 179 & 406 & 388 \\
\hline Social security expenses & 203 & 287 & 452 & 531 & 639 \\
\hline Capital & 815 & 845 & 897 & 1,357 & 1,671 \\
\hline Primary balance & 195 & 986 & 835 & 457 & 1,467 \\
\hline Interest & 240 & 438 & 87 & 97 & 595 \\
\hline Overall balance & -45 & 548 & 749 & 359 & 871 \\
\hline
\end{tabular}


Table 26. Uruguay: Operations of the Public Enterprises and Autonomous Agencies

(In millions of Uruguayan pesos)

\begin{tabular}{|c|c|c|c|c|c|}
\hline & 1994 & 1995 & 1996 & 1997 & $\begin{array}{r}\text { Est. } \\
1998\end{array}$ \\
\hline \multicolumn{6}{|c|}{ III. Petroleum, Alcohol and Cement Company (ANCAP) } \\
\hline Revenues & 3,542 & 5,111 & 7,308 & $\mathbf{8 , 8 8 7}$ & 9,622 \\
\hline Current & 3,542 & 5,111 & 7,308 & 8,887 & 9,622 \\
\hline Sales & 3,484 & 5,028 & 7,189 & 8,743 & 9,466 \\
\hline Other & 58 & 83 & 119 & 144 & 156 \\
\hline Transfers from the central government & 0 & 0 & 0 & 0 & 0 \\
\hline Capital & 0 & 0 & 0 & 0 & 0 \\
\hline Expenditure & 3,831 & 5,037 & 7,354 & $\mathbf{8 , 5 1 5}$ & 9,372 \\
\hline Wages & 210 & 290 & 525 & 535 & 549 \\
\hline Goods and services & 1,574 & 2,229 & 3,546 & 3,725 & 3,545 \\
\hline Taxes & 1,357 & 1,960 & 2,950 & 3,553 & 3,899 \\
\hline Import duties & 17 & 25 & 38 & 45 & 50 \\
\hline Profit taxes & 37 & 53 & 79 & 96 & 105 \\
\hline Discretionary transfers to central government & 153 & 193 & 0 & 313 & 285 \\
\hline Social security expenses & 98 & 139 & 184 & 201 & 282 \\
\hline Capital & 385 & 149 & 33 & 47 & 657 \\
\hline Primary balance & -289 & 74 & -46 & 373 & 250 \\
\hline Interest & 2 & 12 & 0 & 0 & 0 \\
\hline Overall balance & -291 & 62 & -46 & 373 & 250 \\
\hline \multicolumn{6}{|c|}{ IV. National Railways Company (AFE) } \\
\hline Revenues & 125 & 253 & 315 & 325 & 375 \\
\hline Current & 52 & 76 & 107 & 116 & 111 \\
\hline Sales & 33 & 48 & 68 & 74 & 70 \\
\hline Other & 19 & 28 & 39 & 42 & 40 \\
\hline Transfers from the central government & 55 & 177 & 196 & 193 & 190 \\
\hline Capital & 18 & 0 & 12 & 15 & 75 \\
\hline Expenditure & 129 & 244 & 287 & 327 & 341 \\
\hline Wages & 74 & 132 & 168 & 210 & 211 \\
\hline Goods and services & 36 & 92 & 108 & 104 & 108 \\
\hline Taxes & 0 & 0 & 0 & 0 & 0 \\
\hline Import duties & 0 & 0 & 0 & 0 & 0 \\
\hline Profit taxes & 0 & 0 & 0 & 0 & 0 \\
\hline Discretionary transfers to central government & 0 & 0 & 0 & 0 & 0 \\
\hline Social security expenses & 0 & 0 & 0 & 0 & 0 \\
\hline Capital & 19 & 20 & 10 & 12 & 22 \\
\hline Primary balance & -4 & 9 & 29 & -2 & 30 \\
\hline Interest & 2 & 8 & 9 & 9 & 7 \\
\hline Overall balance & -6 & 1 & 20 & -11 & 27 \\
\hline
\end{tabular}


Table 26. Uruguay: Operations of the Public Enterprises and Autonomous Agencies

(In millions of Uruguayan pesos)

\begin{tabular}{|c|c|c|c|c|c|}
\hline & 1994 & 1995 & 1996 & 1997 & $\begin{array}{r}\text { Est. } \\
1998\end{array}$ \\
\hline \multicolumn{6}{|c|}{ V. National Port Administration (ANP) } \\
\hline Revenues & 310 & 385 & 443 & 589 & 666 \\
\hline Current & 310 & 386 & 443 & 589 & 666 \\
\hline Sales & 286 & 357 & 409 & 544 & 615 \\
\hline Other & 24 & 29 & 34 & 45 & 51 \\
\hline Transfers from the central government & 0 & -1 & 0 & 0 & 0 \\
\hline Capital & 0 & 0 & 0 & 0 & 0 \\
\hline Expenditure & 289 & 383 & 450 & 494 & 590 \\
\hline Wages & 139 & 136 & 192 & 209 & 220 \\
\hline Goods and services & 71 & 102 & 102 & 112 & 164 \\
\hline Taxes & 9 & 45 & 20 & 23 & 30 \\
\hline Import duties & 0 & 0 & 0 & 0 & 0 \\
\hline Profit taxes & 0 & 0 & 0 & 0 & 0 \\
\hline Discretionary transfers to central government & 8 & 0 & 0 & 0 & 4 \\
\hline Social security expenses & 47 & 59 & 73 & 85 & 103 \\
\hline Capital & 15 & 40 & 65 & 65 & 69 \\
\hline Primary balance & 21 & 2 & -7 & 95 & 41 \\
\hline Interest & 2 & 7 & 8 & 10 & 7 \\
\hline Overall balance & 19 & -5 & -15 & 86 & 34 \\
\hline \multicolumn{6}{|c|}{ VI. Sanitary Works Company (OSE) } \\
\hline Revenues & 814 & 1,201 & 1,663 & 2,242 & 2,415 \\
\hline Current & 812 & 1,198 & 1,663 & 2,242 & 2,415 \\
\hline Sales & 789 & 1,165 & 1,617 & 2,179 & 2,347 \\
\hline Other & 23 & 33 & 46 & 62 & 67 \\
\hline Transfers from the central government & 2 & 3 & 0 & 0 & 0 \\
\hline Capital & 0 & 0 & 0 & 0 & 0 \\
\hline Expenditure & 815 & 1,158 & 1,846 & 2,316 & 2,390 \\
\hline Wages & 220 & 308 & 493 & 639 & 633 \\
\hline Goods and services & 178 & 315 & 359 & 450 & 515 \\
\hline Taxes & 1 & 2 & 2 & 3 & 3 \\
\hline Import duties & 12 & 22 & 23 & 43 & 48 \\
\hline Profit taxes & 63 & 119 & 126 & 229 & 258 \\
\hline Discretionary transfers to central government & 0 & 0 & 0 & 0 & 0 \\
\hline Social security expenses & 112 & 178 & 289 & 332 & 341 \\
\hline Capital & 230 & 215 & 553 & 621 & 591 \\
\hline Primary balance & -1 & 43 & -182 & -75 & 25 \\
\hline Interest & 8 & 13 & 26 & 31 & 75 \\
\hline Overall balance & -9 & 30 & -208 & -106 & -50 \\
\hline
\end{tabular}


Table 26. Uruguay: Operations of the Public Enterprises and Autonomous Agencies

(In millions of Uruguayan pesos)

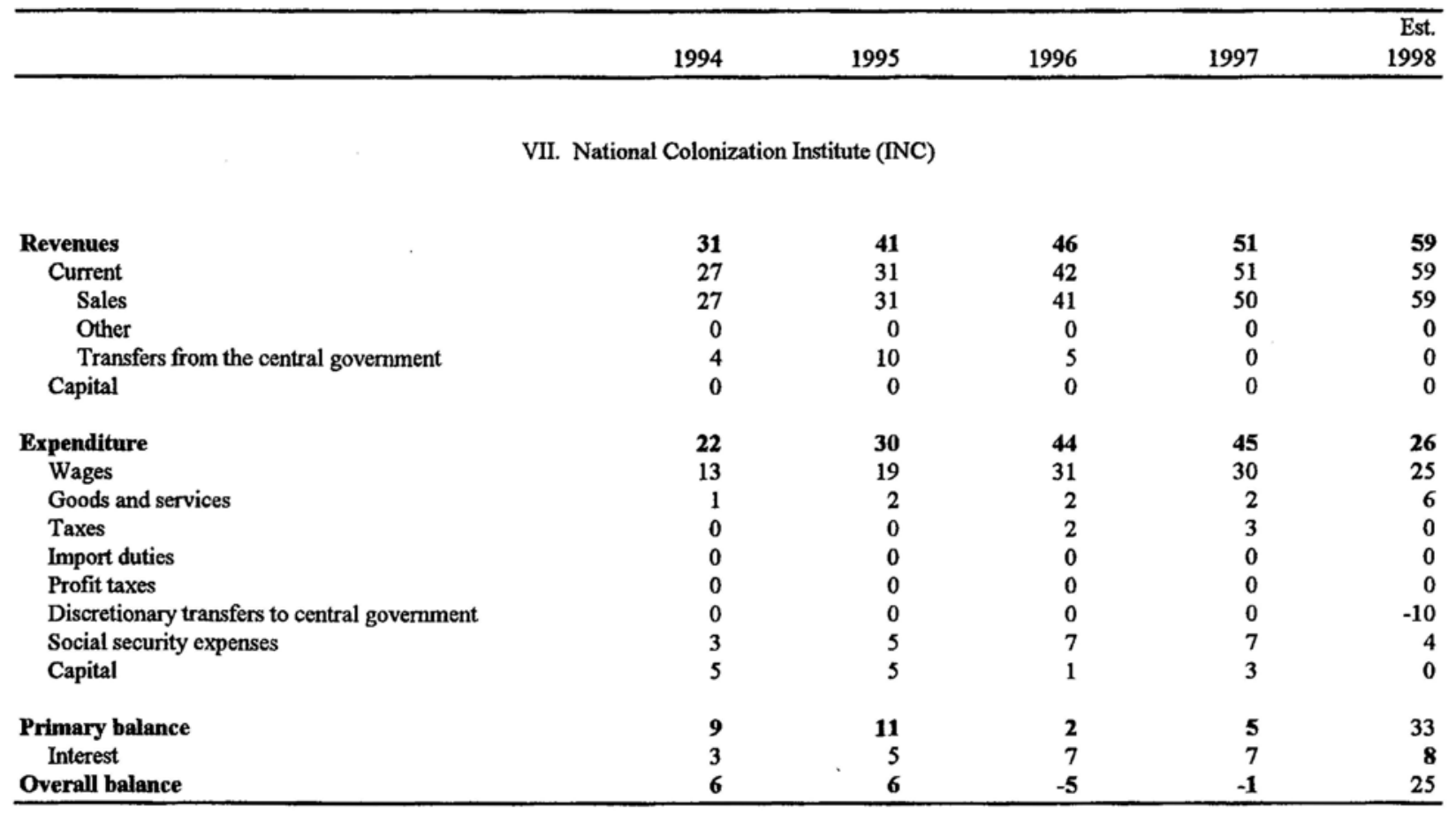

Sources: Office of Planning and Budget; Central Bank of Uruguay; and Fund staff estimates.

1/ Includes changes in stocks.

2/ Privatized in 1995. In 1997 Indemnization outlays amounted Ur \$8 million, included in the overall balance of public enterprises. 
Table 27. Uruguay: Functional Classification of Central Government Expenditure

\begin{tabular}{|c|c|c|c|c|c|}
\hline & 1993 & 1994 & 1995 & 1996 & 1997 \\
\hline \multicolumn{6}{|c|}{ (In millions of Uruguayan pesos) } \\
\hline Total expenditure & 18,103 & 28,717 & 35,390 & 47,914 & 59,743 \\
\hline General public services & 1,575 & 2,420 & 3,228 & 4,863 & 5,978 \\
\hline Defense 1/ & 974 & 2,083 & 1,816 & 2,228 & 2,638 \\
\hline Education & 1,188 & 1,875 & 2,454 & 3,183 & 4,314 \\
\hline Health & 862 & 1,661 & 2,141 & 2,880 & 3,513 \\
\hline Social Security and welfare & 11,219 & 17,397 & 21,169 & 29,614 & 36,061 \\
\hline Housing & 48 & 54 & 636 & 798 & 852 \\
\hline Other social services & 139 & 112 & 144 & 205 & 279 \\
\hline Economic services & 1,294 & 2,011 & 2,041 & 2,510 & 3,712 \\
\hline Unallocated and other purposes & 1,187 & 1,630 & 2,402 & 2,805 & 3,780 \\
\hline Public debt interest & 866 & 1,614 & 2,007 & 1,993 & 3,005 \\
\hline Other & 321 & 16 & 395 & 812 & 775 \\
\hline Adjustment to total expenditure & -383 & -526 & -641 & $-1,172$ & $-1,384$ \\
\hline \multicolumn{6}{|c|}{ (In percent of total) } \\
\hline Total expenditure & 100.0 & 100.0 & 100.0 & 100.0 & 100.0 \\
\hline General public services & 8.7 & 8.4 & 9.1 & 10.1 & 10.0 \\
\hline Defense & 5.4 & 7.3 & 5.1 & 4.6 & 4.4 \\
\hline Education & 6.6 & 6.5 & 6.9 & 6.6 & 7.2 \\
\hline Health & 4.8 & 5.8 & 6.0 & 6.0 & 5.9 \\
\hline Social Security and welfare & 62.0 & 60.6 & 59.8 & 61.8 & 60.4 \\
\hline Housing & 0.3 & 0.2 & 1.8 & 1.7 & 1.4 \\
\hline Other social services & 0.8 & 0.4 & 0.4 & 0.4 & 0.5 \\
\hline Economic services & 7.1 & 7.0 & 5.8 & 5.2 & 6.2 \\
\hline Public debt interest & 4.8 & 5.6 & 5.7 & 4.2 & 5.0 \\
\hline Other expenditure & 1.8 & 0.1 & 1.1 & 1.7 & 1.3 \\
\hline \multicolumn{6}{|c|}{ (In percent of GDP) } \\
\hline \multicolumn{6}{|l|}{ Memorandum items } \\
\hline Expenditure in defense & 1.8 & 2.5 & 1.6 & 1.5 & 1.4 \\
\hline Expenditure in education & 2.2 & 2.3 & 2.2 & 2.1 & 2.3 \\
\hline Expenditure in health & 1.6 & 2.0 & 1.9 & 1.9 & 1.9 \\
\hline
\end{tabular}

Sources: International Monetary Fund, Government Finance Statistics Yearbook.

1/ Excludes extrabudgetary outlays. 
Table 28. Uruguay: Employment in the Public Sector, 1993-98

(In thousands of employees)

\begin{tabular}{lrrrrrr}
\hline & 1993 & 1994 & 1995 & 1996 & 1997 & $19981 /$ \\
\hline & & & & & & \\
Central administration & 103.4 & 105.6 & 101.5 & 97.5 & 96.6 & 94.9 \\
Local administration & 37.3 & 38.5 & 38.2 & 37.4 & 37.4 & 37.4 \\
Official banks & 10.1 & 9.7 & 9.7 & 9.1 & 9.0 & 8.5 \\
Public enterprises & 33.5 & 32.2 & 30.6 & 30.2 & 27.4 & 26.2 \\
Education & 58.0 & 58.0 & 67.9 & 68.3 & 67.8 & 70.1 \\
Legislative & 1.3 & 1.3 & 1.7 & 1.5 & 1.5 & 1.5 \\
Judiciary power & 5.4 & 5.5 & 5.3 & 5.5 & 5.5 & 5.5 \\
BPS & 5.6 & 5.5 & 5.3 & 4.6 & 4.4 & 4.5 \\
& & & & & & \\
Total & 254.4 & 256.2 & 260.1 & 254.2 & 249.6 & 248.6 \\
\hline
\end{tabular}

Source: National Office of the Civil Service.

1/ June 1998. 
Table 29. Selected Monetary and Credit Indicators

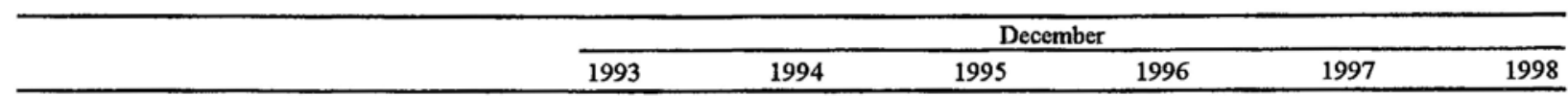

(Percentage change with respect to a year ago)

Monetary agregates $1 /$

Currency

M-1

M-2

M-3*2/

M-3 2/

M-4*2/

M-4 2/

Credit aggregates 3 /

Total banking system credit

Of which: private sector credit

Real money holdings

M-1

M-2

M-3*2/

M-3 2/

$\mathrm{M}-4 * 2 /$

M-4 2/

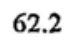

65.9

58.8

41.8

36.0

41.9

36.7

48.3

47.4

$\begin{array}{rr}8.5 & -7.5 \\ 3.8 & -12.0 \\ -7.3 & -2.0 \\ -11.0 & -4.7 \\ -7.2 & -2.0 \\ -10.6 & -4.4\end{array}$

(In percent)

$\begin{array}{llll}30.6 & 21.7 & 23.4 & 10.9 \\ 31.7 & 30.0 & 18.0 & 13.1 \\ 35.5 & 31.3 & 26.7 & 16.8 \\ 27.0 & 37.6 & 34.9 & 20.1 \\ 31.6 & 39.1 & 28.5 & 20.3 \\ 32.1 & 36.9 & 29.1 & 19.4 \\ 33.5 & 37.7 & 27.2 & 19.8 \\ & & & \\ 41.2 & 31.4 & 31.2 & 19.3 \\ 47.9 & 37.6 & 35.5 & 26.7\end{array}$

\section{$-2.7$}

0.1

$-6.2$

$-2.8$

$-2.4$

$-1.4$

\author{
Selected ratios \\ Monetary base to GDP 4/ \\ M1 to GDP 5/ \\ M2 to GDP 5/ \\ M4 to GDP 5/ \\ M4* to GDP 5/
}

$\begin{array}{rr}5.0 & 4.6 \\ 5.2 & 4.8 \\ 8.4 & 7.7 \\ 51.3 & 46.4 \\ 34.3 & 32.2\end{array}$

4.4
4.7
7.1
45.3
27.9

4.2

4.1

3.8

$\begin{array}{lll}4.7 & 4.5 & 4.6\end{array}$

$\begin{array}{lll}7.3 & 7.5 & 7.9\end{array}$

$\begin{array}{lll}7.7 & 7.1 & 7.3\end{array}$

46.0

52.8

Selected interest rates (12-month averages)

\author{
Foreign currency deposits 6/ \\ Foreign currency loans $7 /$ \\ Domestic currency deposits 6/ \\ Domestic currency loans 7/
}

$\begin{array}{rrr}3.1 & 3.4 & 4 . \\ 11.2 & 11.7 & 13.8 \\ 39.4 & 37.0 & 38 . \\ 97.3 & 95.1 & 99 .\end{array}$

4.6
13.8
38.2
99.1

4.8

4.8

$\begin{array}{lll}91.5 & 71.5 & 57.9\end{array}$

Memorandum item

Consumer price inflation $8 /$

52.9

44.1

35.4

24.3

15.2

8.6

Source: Central Bank of Uruguay.

1/ M-1 is defined as currency plus demand deposits; M-2 is defined as M-1 plus time and savings deposits in local currency; M-3* is M-2 plus foreign currency deposits of residents; M-3 is M-3* plus foreign currency deposits of non-residents; M-4* is M-3* plus liabilities of the mortgage bank to the private sector; $\mathrm{M}-4$ is $\mathrm{M}-3$ plus liabilities of the mortgage bank to the private sector.

2/ Foreign currency deposits are valued at end-of-period exchange rates.

3/ Flows of credit in foreign currency are valued at the end-of-period exchange rates.

4/ End of period monetary base including deposits of the rest of the banking system in the Central Bank.

5/ Average monetary aggregates to GDP.

6/ Interest rates on deposits of 30 to 180 day maturity.

$7 /$ Nonprime rate.

8/ End of period. 
Table 30. Uruguay: Summary Accounts of the Financial System 1/

(Annual percentage change)

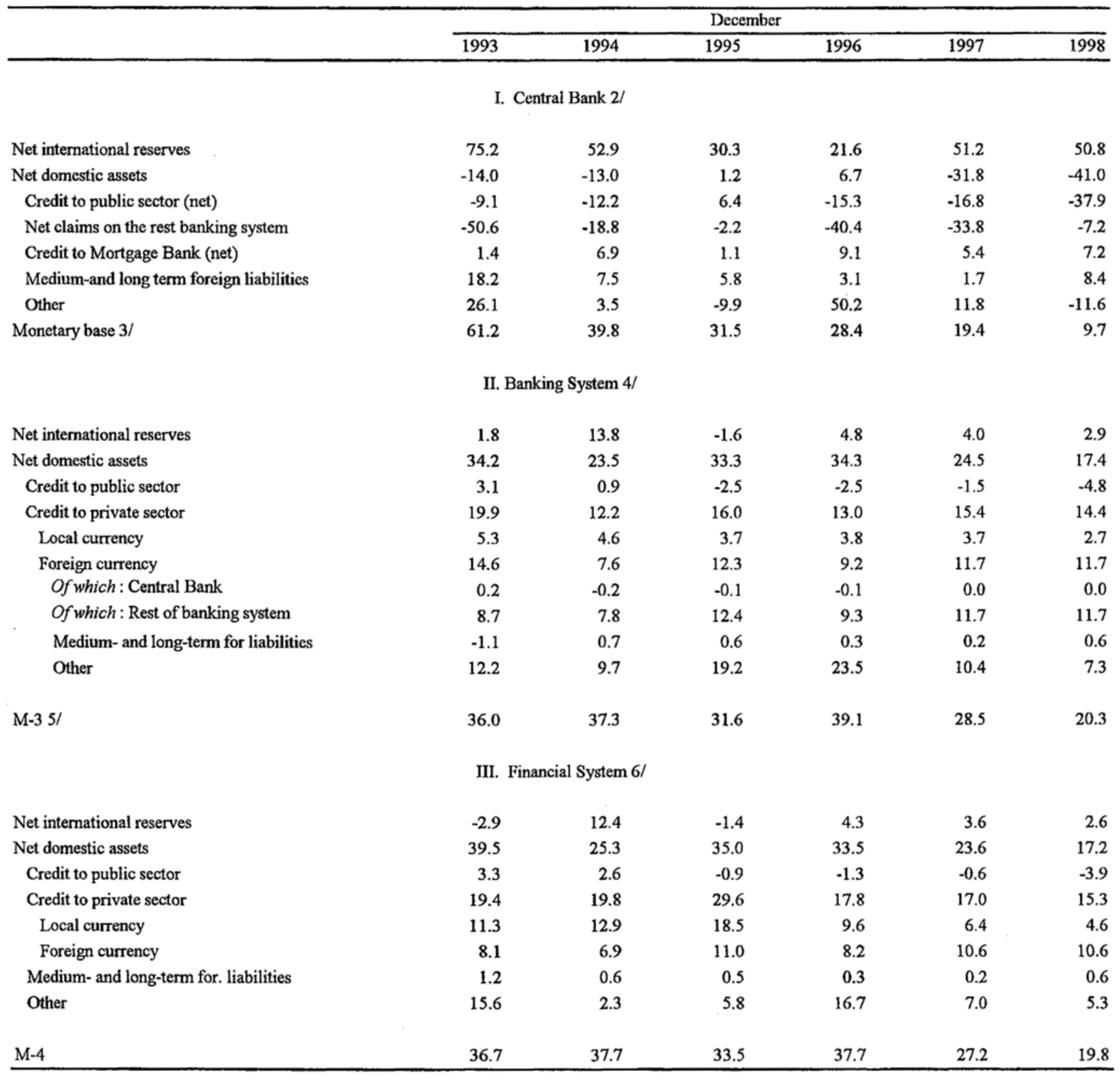

Sources: Central bank of Uruguay; and Fund staff estimates.

1/ Accounts denominated in foreign currency valued at end of period exchange rates.

2/ Changes with respect to the monetary base outstanding at the beginning of the period.

3/ Excludes the Bank of the Republic's vault cash and its deposits at the Central Bank.

4/ Changes with respect to the stock of M-3 at the beginning of the period.

5/ M-3 includes foreign currency deposits of nonresidents.

6/ Changes with respect to the stock of liabilities to the private sector (M-4) at the beginning of the period. 
Table 31. Uruguay: Financial System Credit to the Private Sector 1/

\begin{tabular}{|c|c|c|c|c|c|c|}
\hline & \multicolumn{5}{|c|}{ December } & \multirow[b]{2}{*}{1998} \\
\hline & 1993 & 1994 & 1995 & 1996 & 1997 & \\
\hline \multicolumn{7}{|c|}{ I. Local Currency Credit } \\
\hline \multicolumn{7}{|c|}{ (In millions of Uruguayan pesos) } \\
\hline Total & $7,773.5$ & $11,956.4$ & $20,230.3$ & $25,928.0$ & $31,186.0$ & $36,004.5$ \\
\hline $\mathrm{BCU} 1 /$ & 37.5 & 42.6 & 76.8 & 89.3 & 98.4 & 103.9 \\
\hline BROU 2/ & $1,881.6$ & $2,520.4$ & $3,175.9$ & $3,773.4$ & $4,490.6$ & $6,267.4$ \\
\hline Private banks 3/ & $1,723.3$ & $2,406.1$ & $3,192.1$ & $4,589.8$ & $6,553.7$ & $7,300.1$ \\
\hline BHU 4/ & $4,131.1$ & $6,987.3$ & $13,785.5$ & $17,475.4$ & $20,043.3$ & $22,333.1$ \\
\hline \multicolumn{7}{|c|}{ (12-month percentage change) } \\
\hline Total & 52.6 & 53.8 & 69.2 & 28.2 & 20.3 & 15.5 \\
\hline BCU 1/ & 135.2 & 13.5 & 80.3 & 16.3 & 10.1 & 5.6 \\
\hline BROU 2/ & 42.2 & 33.9 & 26.0 & 18.8 & 19.0 & 39.6 \\
\hline Private banks 3/ & 47.5 & 39.6 & 32.7 & 43.8 & 42.8 & 11.4 \\
\hline BHU 4/ & 59.7 & 69.1 & 97.3 & 26.8 & 14.7 & 11.4 \\
\hline \multicolumn{7}{|c|}{ II. Foreign Currency Credit } \\
\hline \multicolumn{7}{|c|}{ (In millions of Uruguayan pesos) } \\
\hline Total & $13,360.7$ & $19,175.8$ & $29,272.5$ & $40,745.8$ & $55,655.8$ & $71,053.9$ \\
\hline BCU 1/ & 107.4 & 87.6 & 73.7 & 25.6 & 28.0 & 43.9 \\
\hline BROU 2/ & $4,954.2$ & $7,314.9$ & $10,872.1$ & $15,399.6$ & $19,229.0$ & $24,097.9$ \\
\hline Private banks 3/ & $8,264.9$ & $11,717.1$ & $18,239.0$ & $25,144.0$ & $36,030.8$ & $46,382.4$ \\
\hline BHU 4/ & 34.2 & 56.2 & 87.7 & 176.7 & 368.0 & 529.7 \\
\hline \multicolumn{7}{|c|}{ (12-month percentage change) } \\
\hline Total & 48.2 & 43.5 & 52.7 & 39.2 & 36.6 & 27.7 \\
\hline BCU 1/ & 134.8 & -18.4 & -15.9 & -65.3 & 9.4 & 56.9 \\
\hline BROU 2/ & 47.2 & 47.7 & 48.6 & 41.6 & 24.9 & 25.3 \\
\hline Private banks 3/ & 47.8 & 41.8 & 55.7 & 37.9 & 43.3 & 28.7 \\
\hline BHU 4/ & 352.6 & 64.3 & 56.2 & 101.5 & 108.3 & 43.9 \\
\hline \multicolumn{7}{|c|}{ III. Total Credit } \\
\hline \multicolumn{7}{|c|}{ (In millions of Uruguayan pesos) } \\
\hline Total 5/ & $21,134.3$ & $31,132.2$ & $49,502.8$ & $66,673.8$ & $86,841.8$ & $107,058.4$ \\
\hline BCU 1/ & 145.0 & 130.2 & 150.5 & 114.9 & 126.4 & 147.8 \\
\hline BROU 2/ & $6,835.8$ & $9,835.3$ & $14,047.9$ & $19,173.0$ & $23,719.6$ & $30,365.3$ \\
\hline Private banks 3/ & $9,988.2$ & $14,123.2$ & $21,431.1$ & $29,733.8$ & $42,584.5$ & $53,682.5$ \\
\hline BHU 4/ & $4,165.3$ & $7,043.5$ & $13,873.2$ & $17,652.1$ & $20,411.3$ & $22,862.8$ \\
\hline \multicolumn{7}{|c|}{ (12-month percentage change) } \\
\hline Total 5/ & 49.8 & 47.3 & 59.0 & 34.7 & 30.2 & 23.3 \\
\hline BCU 1/ & 134.9 & -10.2 & 15.6 & -23.7 & 10.0 & 17.0 \\
\hline BROU 2/ & 45.8 & 43.9 & 42.8 & 36.5 & 23.7 & 28.0 \\
\hline Private banks 3 / & 47.7 & 41.4 & 51.7 & 38.7 & 43.2 & 26.1 \\
\hline BHU 4/ & 60.6 & 69.1 & 97.0 & 27.2 & 15.6 & 12.0 \\
\hline \multicolumn{7}{|l|}{ Memorandum items } \\
\hline Consumer price index $(1994=100)$ & 69.4 & 100.0 & 135.4 & 168.3 & 193.9 & 210.6 \\
\hline Consumer price inflation (\%) & 52.9 & 44.1 & 35.4 & 24.3 & 15.2 & 8.6 \\
\hline End of year exchange rate & 4,416 & 5,601 & 7,111 & 8,713 & 10,040 & 10,817 \\
\hline
\end{tabular}

Sources: Central Bank of Uruguay; and Fund Staff estimates.

1/ Central Bank of Uruguay

2/ Banco de la República

3/ Includes the intervened banks.

4/ Banco Hipotecario (Mortgage Bank).

5/ Foreign exchange stocks valued at end of period exchange rate.

(C)International Monetary Fund. Not for Redistribution 
Table 32. Uruguay: Financial System Credit to the Private Sector in Real Terms 1/

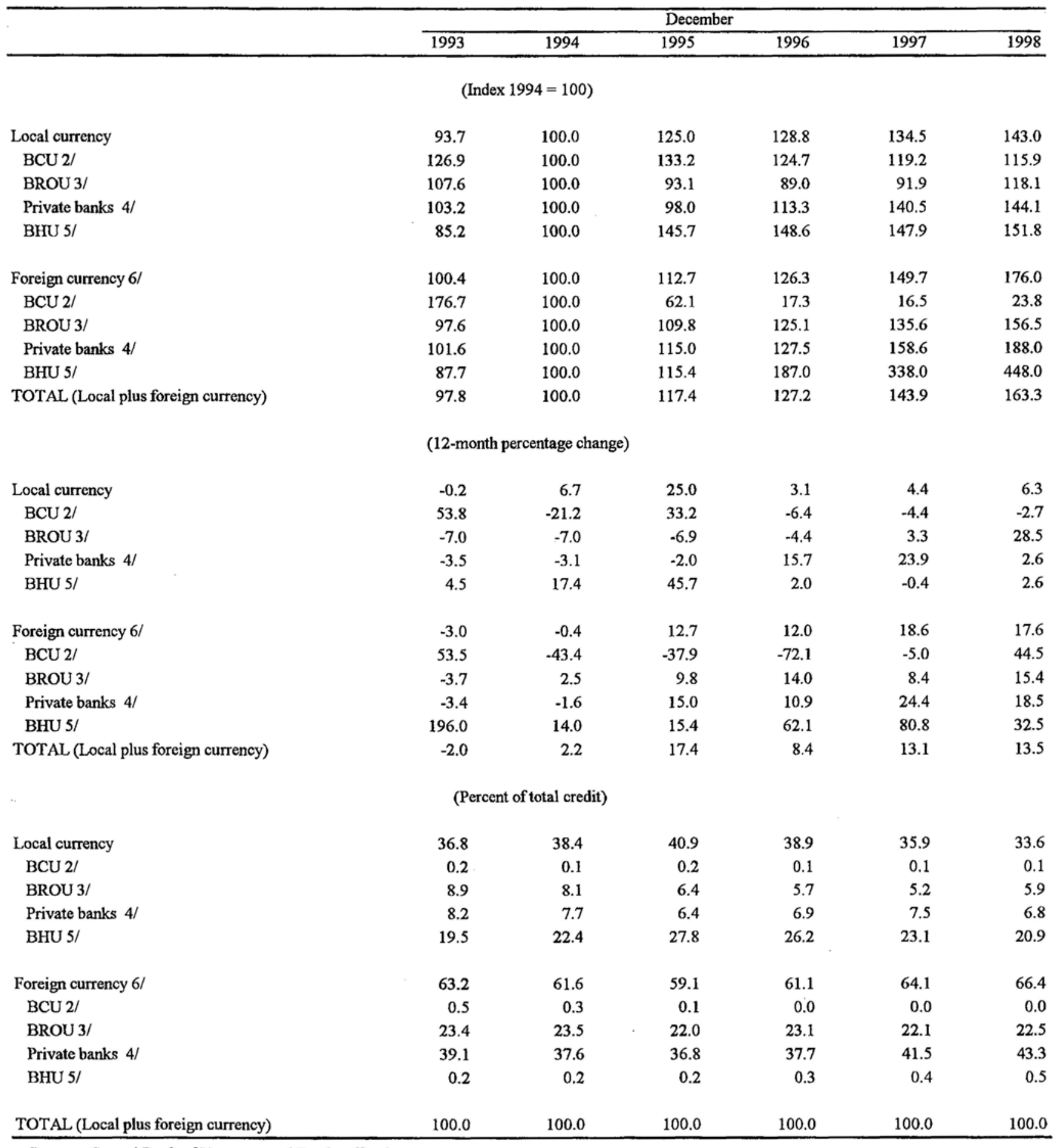

Sources: Central Bank of Uruguay; and Fund staff estimates.

1/ Deflated by the consumer price index.

2/ Central Bank of Uruguay.

3/ Banco de la República.

4/ Includes the intervened banks.

5/ Banco Hipotecario (Mortgage Bank).

6/ Foreign exchange stocks valued at end of period exchange rate. 
Table 33. Uruguay: Composition of Private Sector Financial Assets 1/

\begin{tabular}{|c|c|c|c|c|c|c|}
\hline & \multicolumn{5}{|c|}{ December } & \multirow[b]{2}{*}{1998} \\
\hline & 1993 & 1994 & 1995 & 1996 & 1997 & \\
\hline & \multicolumn{3}{|c|}{ (In millions of Uruguayan pesos) } & & & \\
\hline Total financial assets & 39,451 & 54,281 & 73,688 & 101,243 & 128,811 & 156,539 \\
\hline Banking system & 29,054 & 39,899 & 52,526 & 73,084 & 93,913 & 112,981 \\
\hline Money & 3,762 & 5,017 & 6,609 & 8,590 & 10,136 & 11,469 \\
\hline Currency & 2,313 & 3,314 & 4,327 & 5,265 & 6,498 & 7,208 \\
\hline Demand deposits & 1,449 & 1,703 & 2,282 & 3,325 & 3,638 & 4,261 \\
\hline Quasi-money & 25,292 & 34,882 & 45,917 & 64,494 & 83,777 & 101,513 \\
\hline Local currency deposits & 2,036 & 2,335 & 3,355 & 4,495 & 6,442 & 7,903 \\
\hline Foreign currency deposits & 23,072 & 32,183 & 41,945 & 58,714 & 75,782 & 91,606 \\
\hline held by residents & 13,843 & 20,281 & 25,587 & 35,816 & 47,824 & 57,810 \\
\hline held by nonresidents & 9,229 & 11,902 & 16,359 & 22,899 & 27,958 & 33,795 \\
\hline Other & 184 & 364 & 618 & 1,285 & 1,553 & 2,004 \\
\hline Treasury bills and bonds & 6,680 & 9,348 & 13,219 & 18,846 & 24,103 & 31,183 \\
\hline Letras de regulación monetaria & 375 & 198 & 686 & 0 & 0 & 0 \\
\hline Other financial system & 3,342 & 4,836 & 7,256 & 9,313 & 10,795 & 12,375 \\
\hline Deposits in BHU & 3,320 & 4,685 & 7,012 & 8,914 & 10,359 & 11,900 \\
\hline \multirow[t]{2}{*}{ Mortgage bonds } & 22 & 151 & 244 & 399 & 436 & 475 \\
\hline & \multicolumn{3}{|c|}{ (In percent of total) } & & & \\
\hline Total financial assets & 100.0 & 100.0 & 100.0 & 100.0 & 100.0 & 100.0 \\
\hline Banking system & 73.6 & 73.5 & 71.3 & 72.2 & 72.9 & 72.2 \\
\hline Money & 9.5 & 9.2 & 9.0 & 8.5 & 7.9 & 7.3 \\
\hline Currency & 5.9 & 6.1 & 5.9 & 5.2 & 5.0 & 4.6 \\
\hline Demand deposits & 3.7 & 3.1 & 3.1 & 3.3 & 2.8 & 2.7 \\
\hline Quasi-money & 64.1 & 64.3 & 62.3 & 63.7 & 65.0 & 64.8 \\
\hline Local currency deposits & 5.2 & 4.3 & 4.6 & 4.4 & 5.0 & 5.0 \\
\hline Foreign currency deposits & 58.5 & 59.3 & 56.9 & 58.0 & 58.8 & 58.5 \\
\hline held by residents & 35.1 & 37.4 & 34.7 & 35.4 & 37.1 & 36.9 \\
\hline held by nonresidents & 23.4 & 21.9 & 22.2 & 22.6 & 21.7 & 21.6 \\
\hline Other & 0.5 & 0.7 & 0.8 & 1.3 & 1.2 & 1.3 \\
\hline Treasury bills and bonds & 16.9 & 17.2 & 17.9 & 18.6 & 18.7 & 19.9 \\
\hline Letras de regulación monetaria & 1.0 & 0.4 & 0.9 & 0.0 & 0.0 & 0.0 \\
\hline Other financial system & 8.5 & 8.9 & 9.8 & 9.2 & 8.4 & 7.9 \\
\hline Deposits in BHU & 8.4 & 8.6 & 9.5 & 8.8 & 8.0 & 7.6 \\
\hline Mortgage bonds & 0.1 & 0.3 & 0.3 & 0.4 & 0.3 & 0.3 \\
\hline
\end{tabular}

Sources: Central Bank of Uruguay; and Fund staff estimates.

1/ Foreign currency assets valued at end-of-period exchange rate. 
Table 34. Uruguay: Accounts of the Financial System 1/

(In millions of Uruguayan pesos)

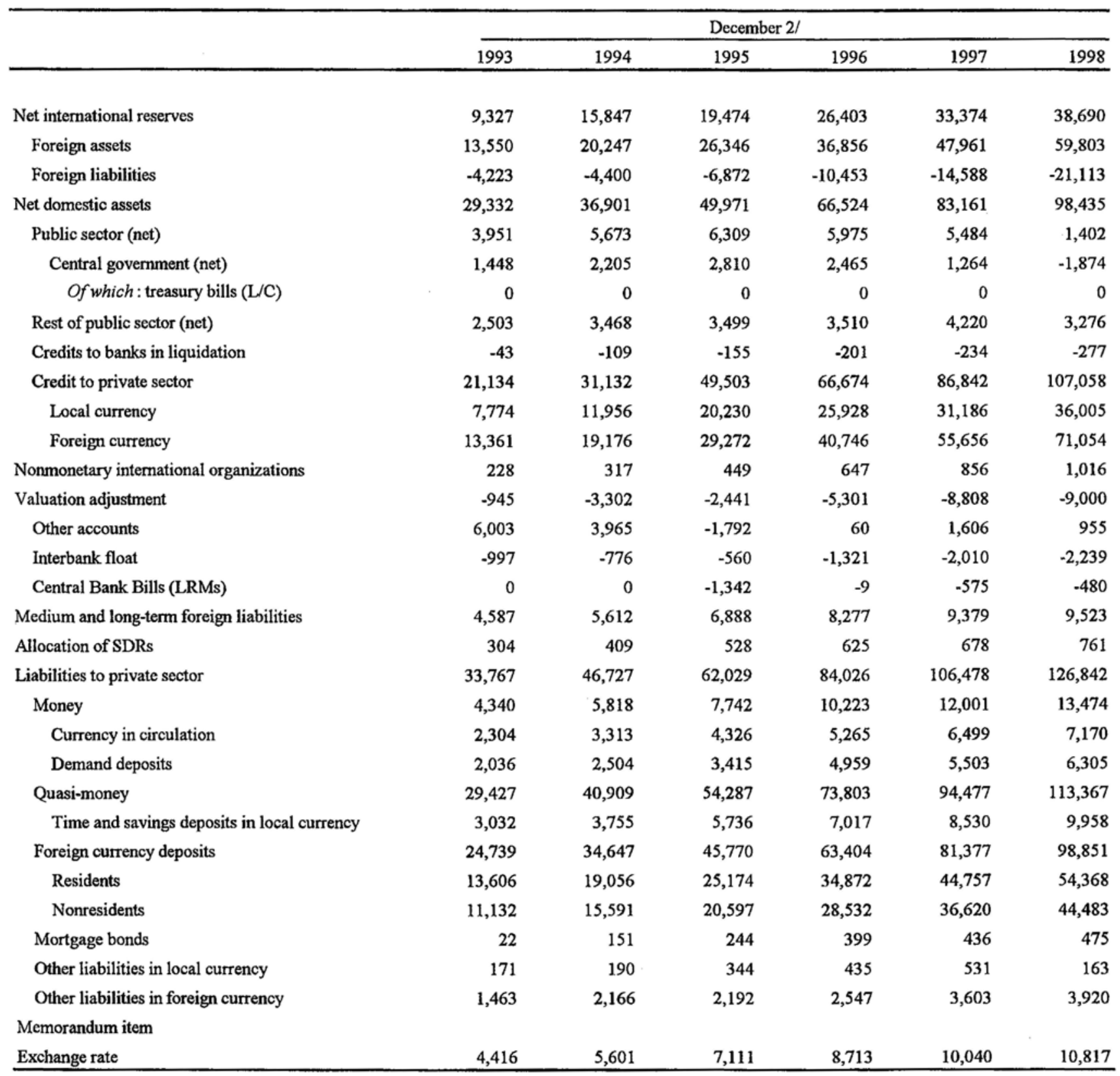

Sources: Central Bank of Uruguay; and Fund staff estimates.

1/ Includes the the Central Bank of Uruguay, the Banco de la República Oriental del Uruguay, the private commercial banks, and the Mortgage Bank. Liabilities to the private sector in this table may differ from the monetary aggregates in Tables 30-31 owing to revisions of data not incorporated in the published balances of financial institutions. Includes other liabilities in local and foreign currencies.

$2 /$ Foreign exchange accounts valued at the exchange rate of the end of the year. 
Table 35. Uruguay: Accounts of the Banking System 1/

(In millions of Uruguayan pesos)

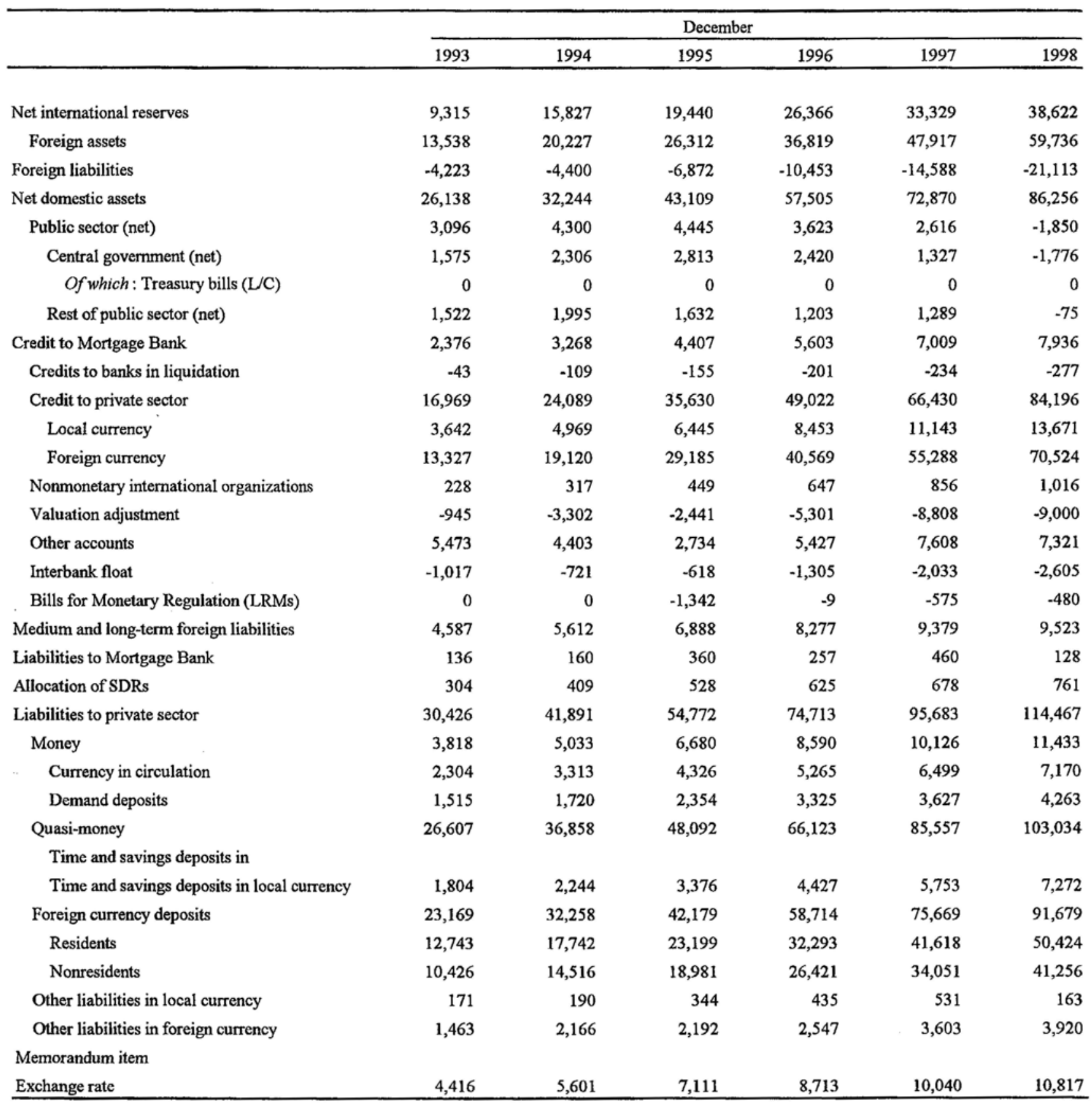

Sources: Central Bank of Uruguay; and Fund staff estimates.

1/ Includes the Central Bank of Uruguay, the Banco de la República Oriental de Uruguay and the private commercial banks.

$2 /$ Foreign exchange accounts valued at the exchange rate of the end of the year. 
Table 36. Uruguay: Accounts of the Central Bank 1/

(In millions of Uruguayan pesos)

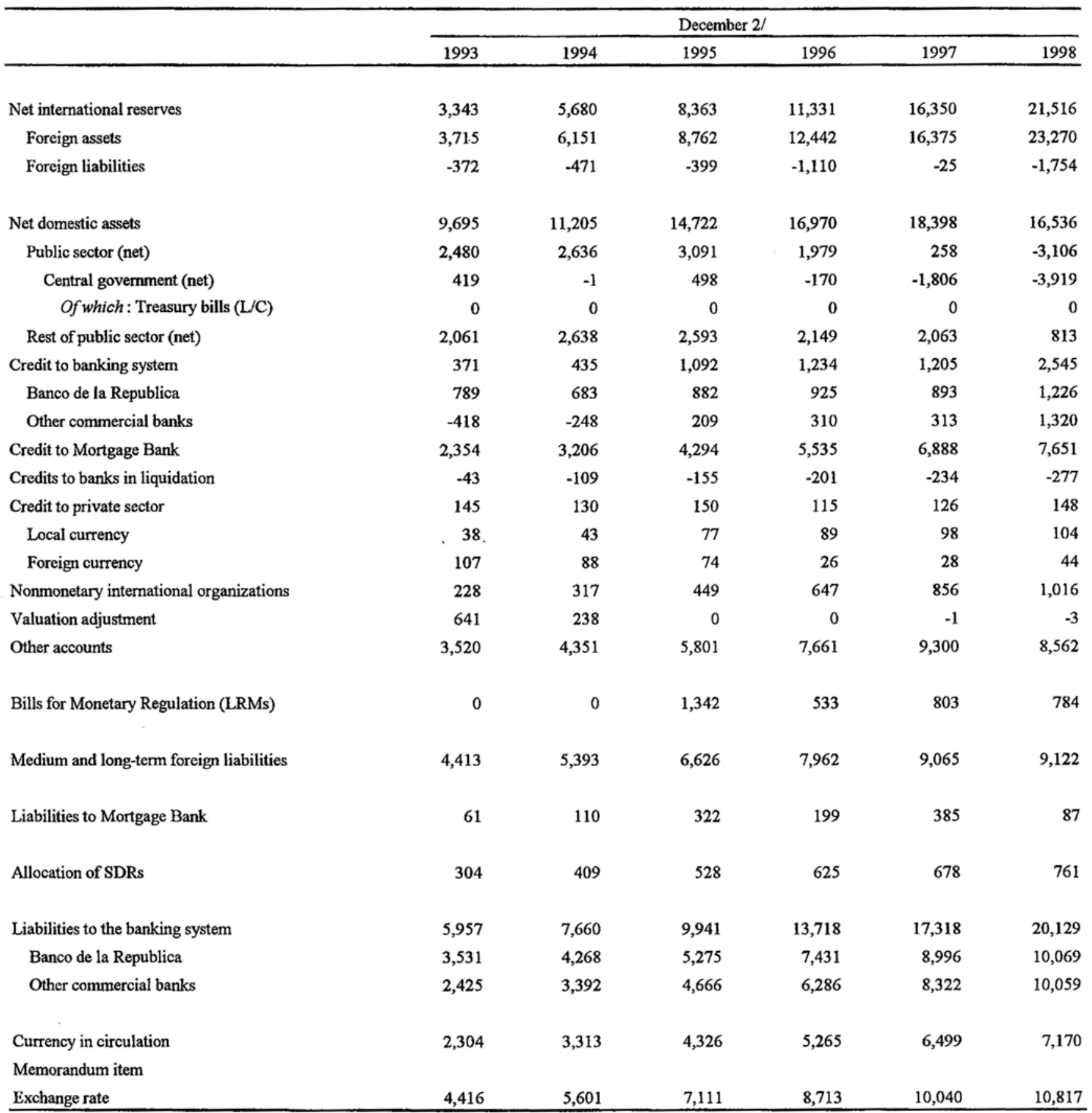

Sources: Central Bank of Uruguay; and Fund staff estimates.

1/ Includes the effect of the external debt reduction operation of February 1991.

2/ Foreign exchange accounts valued at the exchange rate of the end of the year. 
Table 37. Uruguay: Accounts of the Banco de la República Oriental del Uruguay 1/

(In millions of Uruguayan pesos)

\begin{tabular}{|c|c|c|c|c|c|c|}
\hline & \multicolumn{6}{|c|}{ December 2/ } \\
\hline & 1993 & 1994 & 1995 & 1996 & 1997 & 1998 \\
\hline Net international reserves & 2,518 & 2,474 & 3,512 & 4,433 & 5,775 & 6,183 \\
\hline Foreign assets & 2,518 & 2,474 & 3,512 & 4,433 & 5,775 & 6,183 \\
\hline Foreign liabilities & 0 & 0 & 0 & 0 & 0 & 0 \\
\hline Claims on Central Bank & 3,568 & 4,294 & 5,309 & 7,405 & 9,104 & 10,026 \\
\hline Net domestic assets & 3,917 & 6,330 & 9,174 & 12,924 & 16,159 & 20,397 \\
\hline Public sector (net) & $-1,365$ & $-1,397$ & $-1,523$ & $-2,570$ & $-2,916$ & $-4,480$ \\
\hline Central government (net) & -859 & -810 & -609 & $-1,680$ & $-2,182$ & $-3,387$ \\
\hline Of which: treasury bills (L/C) & 0 & 0 & 0 & 0 & 0 & 0 \\
\hline Rest of public sector (net) & -506 & -587 & -914 & -890 & -733 & $-1,093$ \\
\hline Credit to private commercial banks & 18 & 17 & 11 & 26 & 51 & 52 \\
\hline Credit to Mortgage Bank & 0 & 52 & 0 & 0 & 0 & 1 \\
\hline Credit Banks in Liquidation (net) & 0 & 0 & 0 & 0 & 0 & 0 \\
\hline Credit to private sector & 6,836 & 9,835 & 14,048 & 19,173 & 23,720 & 30,365 \\
\hline Local currency & 1,882 & 2,520 & 3,176 & 3,773 & 4,491 & 6,267 \\
\hline Foreign currency & 4,954 & 7,315 & 10,872 & 15,400 & 19,229 & 24,098 \\
\hline Valuation adjustment & -53 & 113 & 43 & 28 & 74 & -160 \\
\hline Other accounts & $-1,520$ & $-2,290$ & $-3,405$ & $-4,257$ & $-4,999$ & $-5,685$ \\
\hline Bills for monetary regulation (LRMs) & 0 & 0 & 0 & 525 & 229 & 304 \\
\hline Medium and long-term foreign liabilities & 174 & 220 & 262 & 315 & 314 & 401 \\
\hline Liabilities to Central Bank & 696 & 480 & 539 & 727 & 894 & 849 \\
\hline Liabilities to private commercial banks & 14 & 18 & 32 & 29 & 145 & 42 \\
\hline Liabilities to Mortgage Bank & 75 & 50 & 38 & 58 & 74 & 41 \\
\hline Liabilities to private sector & 9,044 & 12,331 & 17,124 & 23,632 & 29,610 & 35,272 \\
\hline Demand deposits & 396 & 326 & 461 & 637 & 677 & 815 \\
\hline Time and savings deposits in local currency & 825 & 1,050 & 1,586 & 2,193 & 2,652 & 2,980 \\
\hline Foreign currency deposits & 7,641 & 10,725 & 14,676 & 20,365 & 25,706 & 31,271 \\
\hline Residents & 4,202 & 5,899 & 8,072 & 11,201 & 14,138 & 17,199 \\
\hline Nonresidents & 3,438 & 4,826 & 6,604 & 9,164 & 11,568 & 14,072 \\
\hline Other liabilities in local currency & 134 & 146 & 304 & 379 & 413 & 63 \\
\hline Other liabilities in foreign currency & 48 & 83 & 97 & 59 & 161 & 143 \\
\hline \multicolumn{7}{|l|}{ Memorandum item } \\
\hline Exchange rate & 4,416 & 5,601 & 7,111 & 8,713 & 10,040 & 10,817 \\
\hline
\end{tabular}

Sources: Central Bank of Uruguay; and Fund staff estimates.

1/ Includes the intervened banks.

2/ Foreign exchange accounts valued at the exchange rate of the end of the year. 
Table 38. Uruguay: Accounts of the Private Commercial Banks 1/

(In millions of Uruguayan pesos)

\begin{tabular}{|c|c|c|c|c|c|c|}
\hline & \multicolumn{6}{|c|}{ December 2/ } \\
\hline & 1993 & 1994 & 1995 & 1996 & 1997 & 1998 \\
\hline Net international reserves & 3,454 & 7,673 & 7,564 & 10,601 & 11,204 & 10,924 \\
\hline Foreign assets & 7,305 & 11,602 & 14,038 & 19,944 & 25,767 & 30,283 \\
\hline Foreign liabilities & $-3,851$ & $-3,928$ & $-6,474$ & $-9,343$ & $-14,563$ & $-19,359$ \\
\hline Claims on Central Bank & 1,729 & 2,636 & 3,449 & 4,467 & 5,889 & 5,790 \\
\hline Net domestic assets & 13,948 & 15,912 & 22,297 & 30,737 & 42,452 & 55,353 \\
\hline Public sector (net) & 1,981 & 3,061 & 2,877 & 4,214 & 5,274 & 5,736 \\
\hline Central government (net) & 2,014 & 3,117 & 2,923 & 4,270 & 5,315 & 5,531 \\
\hline Of which: Treasury bills (L/C) & 167 & 21 & 0 & 8 & 0 & 0 \\
\hline Rest of public sector (net) & -33 & -56 & -46 & -55 & -41 & 205 \\
\hline Credit to BROU & 16 & 29 & 22 & 26 & 46 & 43 \\
\hline Credit to Mortgage Bank & 22 & 11 & 113 & 67 & 121 & 284 \\
\hline Credit to private sector & 9,988 & 14,123 & 21,431 & 29,734 & 42,585 & 53,683 \\
\hline Local currency & 1,723 & 2,406 & 3,192 & 4,590 & 6,554 & 7,300 \\
\hline Foreign currency & 8,265 & 11,717 & 18,239 & 25,144 & 36,031 & 46,382 \\
\hline Valuation adjustment & $-1,532$ & $-3,653$ & $-2,484$ & $-5,328$ & $-8,881$ & $-8,836$ \\
\hline Other accounts & 3,474 & 2,342 & 338 & 2,023 & 3,307 & 4,444 \\
\hline Bills for Monetary Regulation (LRMs) & 0 & 0 & 0 & 0 & 0 & 0 \\
\hline Medium and long-term foreign liabilities & 0 & 0 & 0 & 0 & 0 & 0 \\
\hline Liabilities to Central Bank & 0 & 0 & 0 & 0 & 0 & 0 \\
\hline Liabilities to BROU & 53 & -25 & -12 & -11 & -29 & 41 \\
\hline Liabilities to Mortgage Bank & 0 & 0 & 0 & 0 & 0 & 0 \\
\hline Liabilities to private sector & 19,078 & 26,246 & 33,322 & 45,816 & 59,574 & 72,025 \\
\hline Demand deposits & 1,119 & 1,393 & 1,893 & 2,688 & 2,950 & 3,448 \\
\hline Time and savings deposits in local currency & 978 & 1,193 & 1,790 & 2,234 & 3,101 & 4,292 \\
\hline Foreign currency deposits & 15,528 & 21,532 & 27,503 & 38,349 & 49,964 & 60,408 \\
\hline Residents & 8,541 & 11,843 & 15,127 & 21,092 & 27,480 & 33,225 \\
\hline Nonresidents & 6,988 & 9,690 & 12,376 & 17,257 & 22,484 & 27,184 \\
\hline Other liabilities in local currency & 37 & 44 & 40 & 57 & 117 & 100 \\
\hline Other liabilities in foreign currency & 1,415 & 2,083 & 2,096 & 2,488 & 3,442 & 3,777 \\
\hline \multicolumn{7}{|l|}{ Memorandum item } \\
\hline Exchange rate & 4,416 & 5,601 & 7,111 & 8,713 & 10,040 & 10,817 \\
\hline
\end{tabular}

Sources: Central Bank of Uruguay.

$1 /$ Includes the intervened banks.

2/ Foreign exchange accounts valued at the exchange rate of the end of the year. 
Table 39. Uruguay: Accounts of the Mortgage Bank 1/

(In millions of Uruguayan pesos)

\begin{tabular}{|c|c|c|c|c|c|c|}
\hline & \multicolumn{6}{|c|}{ December } \\
\hline & 1993 & 1994 & 1995 & 1996 & 1997 & 1998 \\
\hline Net international reserves & 12 & 20 & 34 & 37 & 44 & 68 \\
\hline Foreign assets & 12 & 20 & 34 & 37 & 44 & 68 \\
\hline Foreign liabilities & 0 & 0 & 0 & 0 & 0 & 0 \\
\hline Claims on banking system & 138 & 78 & 356 & 272 & 547 & 221 \\
\hline Central Bank & 70 & 126 & 355 & 239 & 438 & 150 \\
\hline Of which: currency & 9 & 17 & 33 & 40 & 53 & 58 \\
\hline Banco de la Republica & 68 & 40 & 25 & 33 & 70 & 33 \\
\hline Banca Privada & 0 & -87 & -24 & 0 & 39 & 38 \\
\hline Net domestic assets & 5,550 & 7,979 & 11,211 & 14,637 & 17,278 & 19,750 \\
\hline Public sector (net) & 855 & 1,373 & 1,864 & 2,352 & 2,868 & 3,253 \\
\hline Central government (net) & -127 & -101 & -3 & 45 & -63 & -98 \\
\hline Of which: Treasury bills (L/C) & 7 & 0 & 0 & 0 & 0 & 0 \\
\hline Rest of public sector (net) & 981 & 1,474 & 1,867 & 2,306 & 2,931 & 3,351 \\
\hline Credit to private sector & 4,165 & 7,044 & 13,873 & 17,652 & 20,411 & 22,863 \\
\hline Local currency & 4,131 & 6,987 & 13,786 & 17,475 & 20,043 & 22,333 \\
\hline Foreign currency & 34 & 56 & 88 & 177 & 368 & 530 \\
\hline Other accounts & 530 & -437 & $-4,525$ & $-5,366$ & $-6,002$ & $-6,366$ \\
\hline Liabilities to banking system & 2,358 & 3,241 & 4,345 & 5,634 & 7,074 & 7,663 \\
\hline Central Bank & 2,356 & 3,239 & 4,342 & 5,634 & 7,074 & 7,661 \\
\hline Banco de la Republica & 2 & 2 & 3 & 0 & 0 & 2 \\
\hline Other commercial banks & 0 & 0 & 0 & 0 & 0 & 0 \\
\hline Liabilities to private sector & 3,342 & 4,836 & 7,256 & 9,313 & 10,795 & 12,375 \\
\hline Demand deposits & 522 & 785 & 1,061 & 1,634 & 1,875 & 2,042 \\
\hline \multicolumn{7}{|l|}{ Time and savings deposits in } \\
\hline local currency & 1,229 & 1,511 & 2,360 & 2,590 & 2,776 & 2,687 \\
\hline \multicolumn{7}{|l|}{ Time and savings deposits in } \\
\hline foreign currency & 1,570 & 2,390 & 3,591 & 4,690 & 5,708 & 7,171 \\
\hline Mortgage bonds & 22 & 151 & 244 & 399 & 436 & 475 \\
\hline \multicolumn{7}{|l|}{ Memorandum item } \\
\hline Exchange rate & 4,416 & 5,601 & 7,111 & 8,713 & 10,040 & 10,817 \\
\hline
\end{tabular}

Sources: Central Bank of Uruguay; and Fund staff estimates.

1/ Foreign exchange accounts valued at the exchange rate of the end of the year. 
$-49-$

Table 40. Uruguay: Nominal Short-Term Interest Rates

(Percent per year)

\begin{tabular}{|c|c|c|c|c|c|c|c|c|}
\hline \multirow[b]{3}{*}{$\begin{array}{l}\text { Period } \\
\text { Averages } \\
\end{array}$} & \multicolumn{3}{|c|}{ Deposits } & \multicolumn{4}{|c|}{ Loans } & \multirow[b]{3}{*}{$\begin{array}{l}\text { Open-market } \\
\text { Instruments } 1 /\end{array}$} \\
\hline & \multirow{2}{*}{$\begin{array}{c}\text { In Local Currency } \\
31-180 \\
\text { Days } \\
\end{array}$} & \multicolumn{2}{|c|}{ In Foreign Currency } & \multicolumn{2}{|c|}{ In Local Currency } & \multicolumn{2}{|c|}{$\frac{\text { In Foreign Currency }}{\text { Prime }}$} & \\
\hline & & $\begin{array}{c}\text { Time } \\
\text { Deposits }\end{array}$ & $\begin{array}{c}\text { Sight } \\
\text { Deposits }\end{array}$ & $\begin{array}{l}\text { Prime } \\
\text { Rate }\end{array}$ & Other & $\begin{array}{l}\text { Prime } \\
\text { Rate }\end{array}$ & Other & \\
\hline 1993 & 39.4 & 3.1 & 1.8 & 60.9 & 97.3 & 6.6 & 11.2 & 48.2 \\
\hline 1994 & 37.0 & 3.4 & 1.6 & 59.7 & 98.6 & 7.5 & 11.7 & 44.7 \\
\hline 1995 & 38.2 & 4.6 & 1.7 & 61.7 & 99.1 & 9.0 & 13.8 & 36.6 \\
\hline 1996 & 28.3 & 4.9 & 1.8 & 48.2 & 91.5 & 8.5 & 13.2 & 29.2 \\
\hline 1997 & 19.6 & 4.8 & 1.7 & 39.2 & 71.5 & 8.5 & 12.7 & 23.2 \\
\hline 1998 & 15.1 & 4.9 & 1.7 & 30.6 & 57.9 & 8.5 & 12.4 & 17.0 \\
\hline \multicolumn{9}{|l|}{1993} \\
\hline 1st quarter & 39.3 & 2.9 & 1.8 & 56.7 & 98.6 & 6.5 & 10.8 & 44.5 \\
\hline 2nd quarter & 38.1 & 3.2 & 1.8 & 57.5 & 93.8 & 6.4 & 11.3 & 46.3 \\
\hline 3rd quarter & 40.7 & 3.2 & 1.8 & 65.6 & 96.0 & 6.8 & 11.4 & 56.0 \\
\hline 4th quarter & 39.4 & 3.1 & 1.7 & 60.0 & 100.9 & 6.7 & 11.1 & 46.5 \\
\hline \multicolumn{9}{|l|}{1994} \\
\hline 1st quarter & 37.5 & 3.1 & 1.7 & 55.8 & 97.4 & 6.9 & 11.2 & 40.6 \\
\hline 2nd quarter & 35.0 & 3.3 & 1.6 & 57.3 & 93.6 & 7.3 & 11.2 & 39.6 \\
\hline 3rd quarter & 36.5 & 3.6 & 1.5 & 59.3 & 93.6 & 7.7 & 11.8 & 46.0 \\
\hline 4th quarter & 38.9 & 3.8 & 1.5 & 66.5 & 96.7 & 8.3 & 12.5 & 52.9 \\
\hline \multicolumn{9}{|l|}{1995} \\
\hline 1st quarter & 41.8 & 4.2 & 1.5 & 67.1 & 97.0 & 9.3 & 13.6 & 39.1 \\
\hline 2nd quarter & 37.6 & 4.6 & 1.6 & 66.5 & 101.2 & 9.9 & 14.3 & 35.9 \\
\hline 3rd quarter & 37.1 & 4.4 & 1.7 & 55.4 & 92.4 & 9.1 & 14.2 & 35.8 \\
\hline 4th quarter & 35.8 & 4.7 & 1.7 & 55.4 & 91.2 & 9.2 & 14.0 & 35.5 \\
\hline \multicolumn{9}{|l|}{1996} \\
\hline 1st quarter & 32.4 & 4.9 & 1.8 & 51.1 & 98.6 & 8.4 & 13.8 & 33.9 \\
\hline 2nd quarter & 28.6 & 4.9 & 1.8 & 49.5 & 94.0 & 8.4 & 13.1 & 29.6 \\
\hline 3rd quarter & 26.6 & 4.8 & 1.7 & 47.0 & 88.5 & 8.6 & 12.9 & 27.2 \\
\hline 4th quarter & 25.5 & 4.8 & 1.7 & 45.2 & 85.0 & 8.6 & 12.8 & 26.1 \\
\hline \multicolumn{9}{|l|}{1997} \\
\hline 1st quarter & 21.8 & 4.7 & 1.7 & 45.1 & 79.4 & 8.7 & 12.6 & 25.6 \\
\hline 2nd quarter & 20.4 & 5.0 & 1.7 & 40.3 & 73.9 & 8.4 & 12.8 & 24.8 \\
\hline 3rd quarter & 19.0 & 4.9 & 1.7 & 36.4 & 68.8 & 8.6 & 12.6 & 22.7 \\
\hline 4th quarter & 17.3 & 4.8 & 1.7 & 35.3 & 64.1 & 8.3 & 12.6 & 19.6 \\
\hline \multicolumn{9}{|l|}{1998} \\
\hline 1st quarter & 16.6 & 4.8 & 1.8 & 34.1 & 61.2 & 8.2 & 13.1 & 17.2 \\
\hline 2nd quarter & 14.9 & 5.0 & 1.7 & 32.8 & 61.2 & 8.7 & 12.6 & $\ldots$ \\
\hline 3 rd quarter & 14.4 & 5.0 & 1.7 & 26.7 & 55.4 & 8.4 & 11.7 & 16.9 \\
\hline 4th quarter & 14.5 & 4.9 & 1.7 & 28.8 & 53.9 & 8.7 & 12.3 & $\ldots$ \\
\hline
\end{tabular}

Sources: Central Bank of Uruguay; and Fund staff estimates.

1/ Until the third quarter of 1993, interest rates on central bank bills (Letras de Regulación Monetaria);

thereafter, interest rate on peso-denominated treasury bills of maturity of up to 63 days used in open-market operations.

(C) International Monetary Fund. Not for Redistribution 
Table 41. Uruguay: Private Banks' Required Reserve Ratios on Selected Dates 1/

(In percent of deposits)

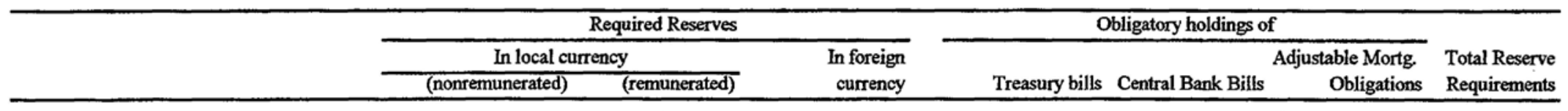

(Situation as of December 31, 1993)

Local currency deposits $2 /$

Sight deposits and time deposits

of less than $\mathbf{3 0}$ days

Time deposits of more than 30 days

but less than 180 days

Time deposits of more than 180 days

Foreign currency deposits 3/

Sight deposits and time deposits

of less than $\mathbf{3 0}$ days

Time deposits of more than 30 days

but less than 180 days

Time deposits of more than 180 days

Credits from correspondents

subject to confirmation

$\begin{array}{ll}10.0 & 10.0 \\ 4.0 & 10 \\ 2.0 & 10\end{array}$

10.0

10.0

10.0

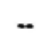

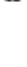

$-$

subject to confirmation

\footnotetext{
but less than 180 days

Time deposits of more than 180 days

Foreign currency deposits 3/

Sight deposits and time deposits of less than $\mathbf{3 0}$ days

Time deposits of more than 30 days but less than 180 days

Time deposits of more than 180 days

Credits from correspondents
}

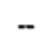

$-$

10.0

11.5

$\begin{array}{lll}- & - & 10.0\end{array}$
$-$

CInternational Monetary Fund. Not for Redistribution

10.0
4.0

CInternational Monetary Fund. Not for Redistribution

$\begin{array}{llll}11.5 & - & - & 21.5 \\ 11.5 & - & - & 21.5 \\ 11.5 & - & - & 15.5 \\ 11.5 & & & \\ & - & - & 21.5\end{array}$

(Situation as of December 31, 1994) 
Table 41. Uruguay: Private Banks' Required Reserve Ratios on Selected Dates 1/

(In percent of deposits)

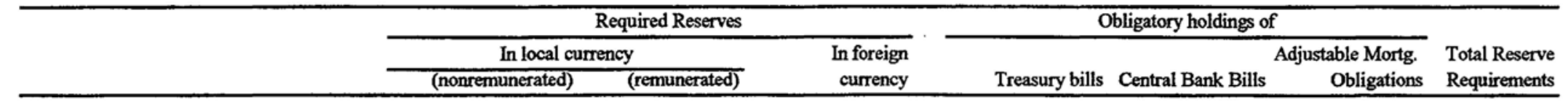

Local currency deposits 2/

Sight deposits and time deposits

of less than 30 days

Time deposits of more than 30 days

but less than 180 days

Time deposits of more than 180 days

Foreign currency deposits $3 /$

Sight deposits and time deposits

of less than 30 days

Time deposits of more than 30 days

but less than 180 days

Time deposits of more than 180 days

Credits from correspondents

subject to confirmation

Local currency deposits 2

Sight deposits and time deposits of less than $\mathbf{3 0}$ days

Time deposits of more than $\mathbf{3 0}$ days

but less than 180 days

Time deposits of more than 180 days

Foreign currency deposits $3 /$

Sight deposits and time deposits

of less than $\mathbf{3 0}$ days

Time deposits of more than $\mathbf{3 0}$ days

but less than 180 days

Time deposits of more than 180 days

Credits from correspondents

subject to confirmation

(Situation as of December 31, 1995)

\begin{tabular}{|c|c|c|c|c|c|c|}
\hline 10.0 & 10.0 & - & -- & - & - & 20.0 \\
\hline 4.0 & 10.0 & - & - & - & - & 14.0 \\
\hline 2.0 & 10.0 & -- & - & - & - & 12.0 \\
\hline- & - & 10.0 & 11.5 & - & - & 21.5 \\
\hline - & - & 10.0 & 11.5 & - & - & 21.5 \\
\hline- & - & 4.0 & 11.5 & - & - & 15.5 \\
\hline & - & 10.0 & 11.5 & - & - & 21.5 \\
\hline
\end{tabular}

(Situation as of December 31, 1996)

10.0

4.0

2.0

$-$

$-$

$-$

$-$
10.0

10.0

10.0
0 
Table 41. Uruguay: Private Banks' Required Reserve Ratios on Selected Dates 1/

(In percent of deposits)

\begin{tabular}{|c|c|c|c|c|c|c|}
\hline \multirow{3}{*}{ - } & \multicolumn{2}{|l|}{ Required Reserves } & \multicolumn{3}{|c|}{ Obligatory holdings of } & \multirow{3}{*}{$\begin{array}{l}\text { Total Reserve } \\
\text { Requirements }\end{array}$} \\
\hline & In local currency & \multirow{2}{*}{$\begin{array}{l}\text { In foreign } \\
\text { currency }\end{array}$} & \multirow[b]{2}{*}{ Treasury bills } & \multirow[b]{2}{*}{ Central Bank Bills } & \multirow{2}{*}{$\begin{array}{r}\text { Adjustable Mortg. } \\
\text { Obligations }\end{array}$} & \\
\hline & (nonremunerated) & & & & & \\
\hline
\end{tabular}

Local currency deposits 2/

(Situation as of December 31, 1997)

Sight deposits and time deposits

of less than $\mathbf{3 0}$ days

Time deposits of more than 30 days

but less than 180 days

Time deposits of more than 180 days

10.0

4.0

2.0

\section{of less than $\mathbf{3 0}$ days}

Time deposits of more than 30 days

but less than 180 days

Time deposits of more than 180 days

Credits from correspondents

subject to confirmation

Local currency deposits 2

Sight deposits and time deposits

of less than $\mathbf{3 0}$ days

Time deposits of more than $\mathbf{3 0}$ days

but less than 180 days

Time deposits of more than 180 days

Foreign currency deposits 3/

Sight deposits and time deposits

of less than $\mathbf{3 0}$ days

Time deposits of more than $\mathbf{3 0}$ days

but less than 180 days

Time deposits of more than 180 days

Credits from correspondents

subject to confirmation

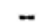

$-$

-

(Situation as of December 31, 1998)

Source: Central Bank of Uruguay.

10.0

10.0

$\begin{array}{rr}- & 10.0 \\ - & 10.0 \\ - & 4.0\end{array}$

10.0

10.0

10.0

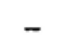

$4.0 \quad 10.0$

$2.0 \quad 10.0$

10.0

-

10.0

4.0

\section{0}

11.5

11.5

11.5

11.5

$-$

$-$

$-$

20.0

$-$

14.0

12.0

$-$

$-$

20.0

11.5

11.5

11.5

$-$

-

14.0

12.0

1/ Excluding financial houses, which are subject to reserve requirements very similar to those of commercial banks.

2/ Applies to all liabilities in local currency to the private sector.

3/ Excludes foreign currency deposits of nonresidents that are used to provide credit to nonresidents (offshore operations). It also excludes public securities after December 1997

C)International Monetary Fund. Not for Redistribution 
Table 42. Uruguay: Summary Balance of Payments $1 /$

\begin{tabular}{|c|c|c|c|c|c|c|}
\hline & 1993 & 1994 & 1995 & 1996 & 1997 & $\begin{array}{l}\text { Prel. } \\
1998\end{array}$ \\
\hline \multicolumn{7}{|c|}{ (In millions of U.S. dollars) } \\
\hline Current account balance & -353 & -439 & -213 & -233 & -287 & -400 \\
\hline Trade Balance & -473 & -686 & -563 & -687 & -704 & -762 \\
\hline Exports, f.o.b. & 1,645 & 1,913 & 2,148 & 2,449 & 2,793 & 2,832 \\
\hline Imports, f.o.b. & $-2,118$ & $-2,600$ & $-2,711$ & $-3,135$ & $-3,498$ & $-3,594$ \\
\hline Nonfactor services (net) & 275 & 450 & 502 & 560 & 536 & 480 \\
\hline \multicolumn{7}{|l|}{ Of which: } \\
\hline Travel receipts & 447 & 632 & 611 & 717 & 759 & 695 \\
\hline Factor services (net) & -193 & -243 & -227 & -189 & -193 & -185 \\
\hline Transfers (net) & 38 & 41 & 76 & 83 & 74 & 67 \\
\hline Financial account & 435 & 672 & 404 & 225 & 539 & 677 \\
\hline Foreign direct investment & 173 & 154 & 157 & 137 & 113 & 164 \\
\hline Portfolio investment & 29 & 158 & 192 & 276 & 174 & 415 \\
\hline Other investment & 232 & 360 & 55 & -189 & 252 & 98 \\
\hline Commercial credit & 21 & 75 & -47 & -17 & 44 & 22 \\
\hline Net assets & 212 & 285 & 102 & -172 & 208 & 76 \\
\hline Errors and omissions & 102 & 5 & 18 & 153 & 79 & 85 \\
\hline Changes in gold valuation & 31 & 0 & -209 & 0 & 0 & 0 \\
\hline Change in international reserves $(-)$ & -214 & -238 & 0 & -144 & -330 & -362 \\
\hline \multicolumn{7}{|c|}{ (In percent, unless otherwise indicated) } \\
\hline \multicolumn{7}{|l|}{ Memorandum items } \\
\hline Current account/GDP & -2.7 & -2.9 & -1.2 & -1.3 & -1.5 & -1.9 \\
\hline Financial account/GDP & 3.3 & 4.4 & 2.4 & 1.2 & 2.8 & 3.2 \\
\hline Public external debt/GDP & 26.6 & 27.9 & 25.7 & 25.4 & 24.9 & 25.3 \\
\hline $\begin{array}{l}\text { Gross official reserves (in months of } \\
\text { imports of goods and services) }\end{array}$ & 5.4 & 5.2 & 6.1 & 5.8 & 5.7 & 6.9 \\
\hline
\end{tabular}

Sources: Central Bank of Uruguay; and Fund staff estimates.

1/ From 1995 onward, the figures in the current account and private sector capital flows incorporate new survey information which permits the identification of certain exports of services in the current account that in prior years were subsumed in private sector capital inflows. Therefore, the figures prior to 1995 and those thereafter are not strictly comparable. 
Table 43. Uruguay: Balance of Payments, 1993-98

(In millions of U.S. dollars)

\begin{tabular}{|c|c|c|c|c|c|c|c|c|c|}
\hline & \multicolumn{3}{|c|}{1993} & \multicolumn{3}{|c|}{1994} & \multicolumn{3}{|c|}{1995} \\
\hline & Credit & Debit & Net & Credit & Debit & Net & Credit & Debit & Net \\
\hline Current account balance & $2,955.8$ & $3,309.1$ & -353.3 & $3,579.4$ & $4,018.1$ & -438.7 & $3,995.0$ & $4,207.6$ & -212.6 \\
\hline Goods f.o.b. & $1,645.3$ & $2,118.3$ & -473.0 & $1,913.3$ & $2,599.6$ & -686.3 & $2,147.5$ & $2,710.6$ & -563.1 \\
\hline Merchandise & $1,645.3$ & $2,118.3$ & -473.0 & $1,913.3$ & $2,599.6$ & -686.3 & $2,105.9$ & $2,678.3$ & .572 .4 \\
\hline Other goods & 0.0 & 0.0 & 0.0 & 0.0 & 0.0 & 0.0 & 41.6 & 32.3 & 9.3 \\
\hline Services & $1,015.2$ & 740.1 & 275.1 & $1,334.9$ & 885.4 & 449.5 & $1,359.2$ & 857.7 & 501.5 \\
\hline Transport & 354.0 & 374.7 & -20.7 & 392.7 & 376.2 & 16.5 & 399.8 & 376.3 & 23.5 \\
\hline Travel & 446.8 & 128.7 & 318.1 & 632.2 & 234.4 & 397.8 & 610.9 & 236.6 & 374.3 \\
\hline Other services & 214.4 & 236.7 & -22.3 & 310.0 & 274.8 & 35.2 & 348.5 & 244.8 & 103.7 \\
\hline Factor income & 249.9 & 442.9 & -193.0 & 282.0 & 525.1 & -243.1 & 404.3 & 631.3 & -227.0 \\
\hline Transfers & 45.4 & 7.8 & 37.6 & 49.2 & 8.0 & 41.2 & 84.0 & 8.0 & 76.0 \\
\hline Financial account & 843.2 & 408.7 & 434.5 & $1,000.3$ & 328.4 & 671.9 & $1,316.8$ & 913.0 & 403.8 \\
\hline Foreign direct investment & 172.8 & 0.0 & 172.8 & 154.0 & 0.0 & 154.0 & 156.6 & 0.0 & 156.6 \\
\hline Portfolio investment & 158.3 & 129.0 & 29.3 & 178.0 & 19.8 & 158.2 & 192.3 & 0.0 & 192.3 \\
\hline Treasury bills & 158.3 & 0.0 & 158.3 & 178.0 & 0.0 & 178.0 & 183.0 & 0.0 & 183.0 \\
\hline Central bank bills & 0.0 & 129.0 & -129.0 & 0.0 & 19.8 & -19.8 & 9.3 & 0.0 & 9.3 \\
\hline Other & 0.0 & 0.0 & 0.0 & 0.0 & 0.0 & 0.0 & 0.0 & 0.0 & 0.0 \\
\hline Other investment & 512.1 & 279.7 & 232.4 & 668.3 & 308.6 & 359.7 & 967.9 & 913.0 & 54.9 \\
\hline Commercial credits & 27.1 & 6.5 & 20.6 & 75.2 & 0.0 & 75.2 & 0.0 & 47.3 & -47.3 \\
\hline General government & 0.0 & 6.5 & -6.5 & 36.0 & 0.0 & 36.0 & 0.0 & 42.8 & -42.8 \\
\hline Nonfinancial private sector & 27.1 & 0.0 & 27.1 & 39.2 & 0.0 & 39.2 & 0.0 & 4.5 & -4.5 \\
\hline Net assets & 485.0 & 273.2 & 211.8 & 593.1 & 308.6 & 284.5 & 967.9 & 865.7 & 102.2 \\
\hline Monetary authorities & 66.9 & 0.0 & 66.9 & 4.0 & 0.0 & 4.0 & 71.7 & 0.0 & 71.7 \\
\hline General government & 38.8 & 0.0 & 38.8 & 134.0 & 0.0 & 134.0 & 61.4 & 0.0 & 61.4 \\
\hline Banks & 374.2 & 273.2 & 101.0 & 455.1 & 276.9 & 178.2 & 827.0 & 831.4 & -4.4 \\
\hline BROU & 86.6 & 29.9 & 56.7 & 136.0 & 2.4 & 133.6 & 43.4 & 60.9 & -17.5 \\
\hline BHU & 32.1 & 0.0 & 32.1 & 0.0 & 6.9 & -6.9 & 0.0 & 3.1 & -3.1 \\
\hline Private banks & 255.5 & 243.3 & 12.2 & 319.1 & 267.6 & 51.5 & 783.6 & 767.4 & 16.2 \\
\hline Other sectors & 5.1 & 0.0 & 5.1 & 0.0 & 31.7 & -31.7 & 7.8 & 34.3 & -26.5 \\
\hline Errors and omissions & 101.6 & 0.0 & 101.6 & 4.7 & 0.0 & 4.7 & 18.0 & 0.0 & 18.0 \\
\hline Adjustment in the value of gold & 30.7 & 0.0 & 30.7 & 0.0 & 0.0 & 0.0 & 0.1 & 209.4 & -209.3 \\
\hline Change in international reserves & 87.7 & 301.1 & -213.5 & 13.9 & 251.9 & -237.9 & 0.0 & 209.4 & 0.1 \\
\hline Liabilities & 0.0 & 26.0 & -26.0 & 13.5 & 8.5 & 5.0 & 0.0 & 47.0 & -47.0 \\
\hline Assets & 87.7 & 275.1 & -187.4 & 0.4 & 243.4 & -243.0 & 0.0 & 162.4 & -162.4 \\
\hline
\end{tabular}


Table 43. Uruguay: Balance of Payments, 1993-98

\begin{tabular}{|c|c|c|c|c|c|c|c|c|c|}
\hline & \multicolumn{3}{|c|}{1996} & \multicolumn{3}{|c|}{1997} & \multicolumn{3}{|c|}{1998} \\
\hline & Credit & Debit & Net & Credit & Debit & $\overline{\text { Net }}$ & Credit & Debit & $\overline{\mathrm{Net}}$ \\
\hline Current account balance & $4,398.4$ & $4,631.8$ & -233.4 & $4,847.5$ & $5,134.9$ & -287.4 & $4,910.4$ & $5,310.4$ & -400.0 \\
\hline Goods f.o.b. & $2,448.5$ & $3,135.4$ & -686.9 & $2,793.1$ & $3,497.5$ & -704.4 & $2,832.3$ & $3,594.2$ & -761.9 \\
\hline Merchandise & $2,397.2$ & $3,109.7$ & .712 .5 & $2,729.5$ & $3,477.6$ & -748.1 & $2,768.7$ & $3,574.3$ & -805.6 \\
\hline Other goods & 51.3 & 25.7 & 25.6 & 63.6 & 19.9 & 43.7 & 63.6 & 19.9 & 43.7 \\
\hline Services & $1,398.7$ & 839.0 & 559.7 & $1,424.1$ & 888.6 & 535.5 & $1,392.9$ & 912.8 & 480.1 \\
\hline Transport & 394.1 & 445.9 & -51.8 & 342.8 & 425.9 & -83.1 & 340.0 & 421.0 & -81.0 \\
\hline Travel & 716.8 & 192.0 & 524.8 & 759.3 & 264.1 & 495.2 & 694.9 & 265.0 & 429.9 \\
\hline Other services & 287.8 & 201.1 & 86.7 & 322.0 & 198.6 & 123.4 & 358.0 & 226.8 & 131.2 \\
\hline Factor income & 460.5 & 649.2 & -188.7 & 547.3 & 740.0 & -192.7 & 602.4 & 787.4 & -185.0 \\
\hline Transfers & 90.7 & 8.2 & 82.5 & 83.0 & 8.8 & 74.2 & 82.8 & 16.0 & 66.8 \\
\hline Financial account & 514.6 & 289.9 & 224.7 & 835.5 & 296.7 & 538.8 & 776.7 & 100.2 & 676.5 \\
\hline Foreign direct investment & 136.8 & 0.0 & 136.8 & 126.4 & 13.2 & 113.2 & 163.8 & 0.0 & 163.8 \\
\hline Portfolio investment & 284.6 & 8.2 & 276.4 & 298.7 & 124.7 & 174.0 & 414.8 & 0.0 & 414.8 \\
\hline Treasury bills & 284.6 & 0.0 & 284.6 & 298.7 & 0.0 & 298.7 & 305.2 & 0.0 & 305.2 \\
\hline Central bank bills & 0.0 & 8.2 & -8.2 & 0.0 & 124.7 & -124.7 & 109.6 & 0.0 & 109.6 \\
\hline Other & 0.0 & 0.0 & 0.0 & 0.0 & 0.0 & 0.0 & 0.0 & 0.0 & 0.0 \\
\hline Other investment & 93.2 & 281.7 & -188.5 & 410.4 & 158.8 & 251.6 & 198.1 & 100.2 & 97.9 \\
\hline Commercial credits & 0.0 & 16.8 & -16.8 & 87.6 & 43.7 & 43.9 & 22.3 & 0.0 & 22.3 \\
\hline General government & 0.0 & 10.9 & -10.9 & 0.0 & 43.7 & -43.7 & 6.1 & 0.0 & 6.1 \\
\hline Nonfinancial private sector & 0.0 & 5.9 & -5.9 & 87.6 & 0.0 & 87.6 & 16.2 & 0.0 & 16.2 \\
\hline Net assets & 93.2 & 264.9 & .171 .7 & 322.8 & 115.1 & 207.7 & 175.8 & 100.2 & 75.6 \\
\hline Monetary authorities & 0.0 & 79.6 & -79.6 & 35.6 & 16.9 & 18.7 & 0.0 & 16.6 & -16.6 \\
\hline General government & 31.2 & 0.0 & 31.2 & 170.1 & 0.0 & 170.1 & 142.4 & 0.0 & 142.4 \\
\hline Banks & 11.9 & 167.5 & -155.6 & 117.1 & 88.4 & 28.7 & 33.4 & 36.2 & -2.8 \\
\hline BROU & 8.0 & 0.0 & 8.0 & 0.0 & 86.9 & -86.9 & 0.0 & 28.9 & -28.9 \\
\hline $\mathrm{BHU}$ & 3.9 & 0.0 & 3.9 & 0.0 & 1.5 & -1.5 & 0.0 & 7.3 & -7.3 \\
\hline Private banks & 0.0 & 167.5 & -167.5 & 117.1 & 0.0 & 117.1 & 33.4 & 0.0 & 33.4 \\
\hline Other sectors & 50.1 & 17.8 & 32.3 & 0.0 & 9.8 & -9.8 & 0.0 & 47.4 & -47.4 \\
\hline Errors and omissions & 152.5 & 0.0 & 152.5 & 79.0 & 0.0 & 79.0 & 85.4 & 0.0 & 85.4 \\
\hline Adjustment in the value of gold & 0.0 & 0.0 & 0.0 & 0.0 & 0.0 & 0.0 & 0.0 & 0.0 & 0.0 \\
\hline Change in international reserves & 0.0 & 143.8 & -143.8 & 0.0 & 330.4 & -330.4 & 160.1 & 522.0 & -361.9 \\
\hline Liabilities & 0.0 & 25.2 & -25.2 & 0.0 & 9.3 & -9.3 & 160.1 & 0.0 & 160.1 \\
\hline Assets & 0.0 & 118.6 & -118.6 & 0.0 & 321.1 & -321.1 & 0.0 & 522.0 & .522 .0 \\
\hline
\end{tabular}

Source: Central Bank of Uruguay. 
Table 44. Uruguay: Value, Volume, and Unit Value of Merchandise Trade

(Percentage change)

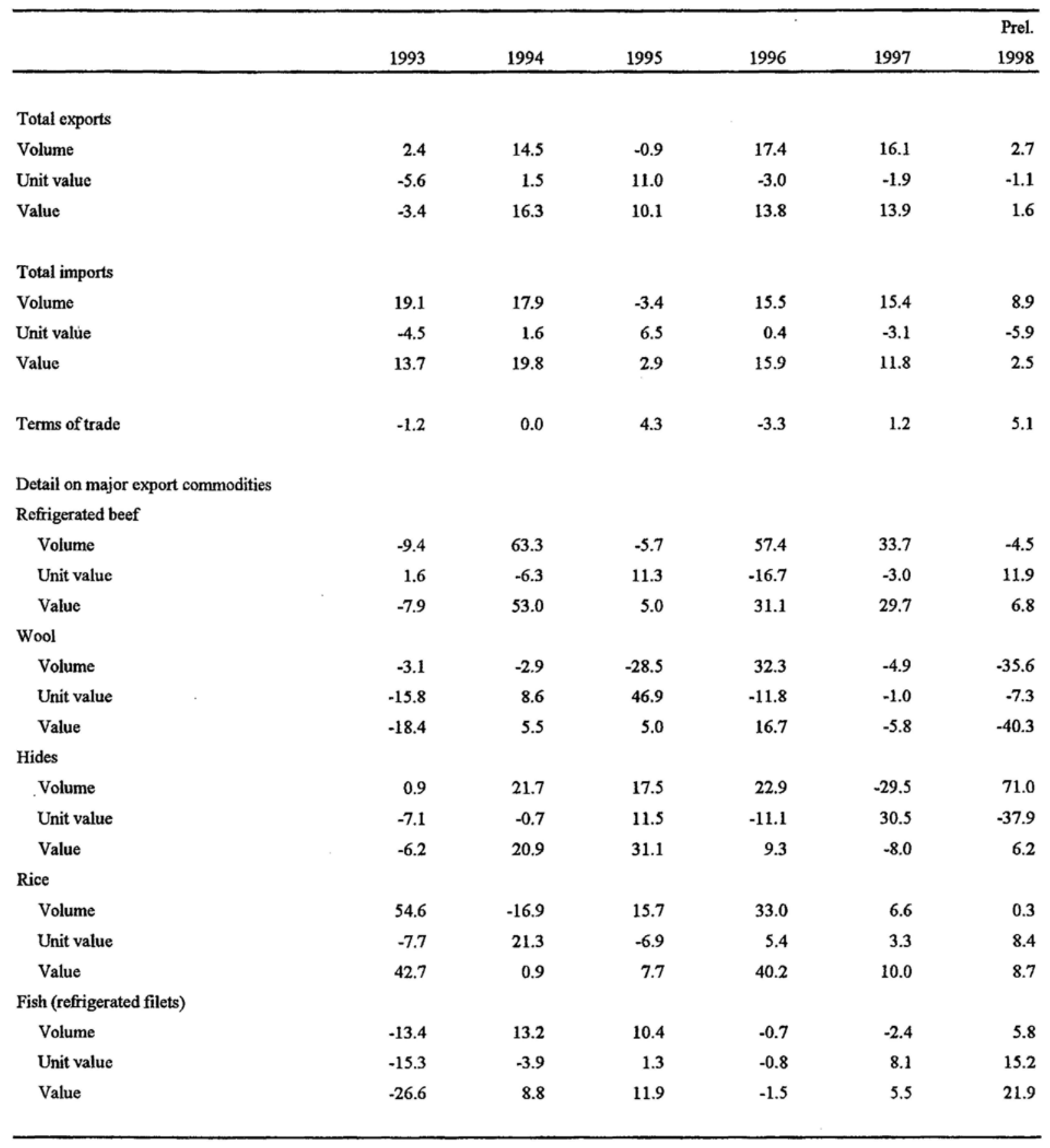

Source: Central Bank of Uruguay. 
Table 45. Uruguay: Summary of Merchandise Exports, f.o.b. 1/

\begin{tabular}{|c|c|c|c|c|c|c|}
\hline & 1993 & 1994 & 1995 & 1996 & 1997 & $\begin{array}{l}\text { Prel. } \\
1998 \\
\end{array}$ \\
\hline \multicolumn{7}{|c|}{ (In millions of U.S. dollars) } \\
\hline Total exports & $1,645.3$ & $1,913.3$ & $2,105.9$ & $2,397.2$ & $2,729.5$ & $2,768.7$ \\
\hline Traditional exports & 432.5 & 561.9 & 648.7 & 712.2 & 844.8 & 726.3 \\
\hline Wool & 217.7 & 229.5 & 241.1 & 290.8 & 276.7 & 158.2 \\
\hline Meat & 167.5 & 236.6 & 242.3 & 322.2 & 408.8 & 433.4 \\
\hline Other & 47.3 & 95.8 & 165.3 & 99.2 & 159.3 & 134.7 \\
\hline Nontraditional exports & $1,212.8$ & $1,351.4$ & $1,457.2$ & $1,685.0$ & $1,884.7$ & $2,042.4$ \\
\hline Rice & 150.1 & 151.3 & 163.0 & 228.5 & 251.5 & 273.4 \\
\hline Fish and shellfish & 69.6 & 75.7 & 87.0 & 89.6 & 100.3 & 116.9 \\
\hline Chemical products & 63.1 & 76.3 & 86.1 & 92.8 & 112.5 & 120.7 \\
\hline Textile manufactures & 170.7 & 162.9 & 180.3 & 185.2 & 203.3 & 191.6 \\
\hline Shoes and others & 25.6 & 21.9 & 17.8 & 23.2 & 21.3 & 17.3 \\
\hline Leather manufactures & 30.3 & 25.6 & 24.6 & 24.0 & 14.8 & 10.5 \\
\hline Other nontraditional & 703.4 & 837.7 & 898.4 & $1,041.7$ & $1,181.0$ & $1,312.0$ \\
\hline \multicolumn{7}{|c|}{ (Percentage change) } \\
\hline \multicolumn{7}{|l|}{ Memorandum items } \\
\hline Exports & -3.4 & 16.3 & 10.1 & 13.8 & 13.9 & 1.4 \\
\hline Traditional exports & -15.9 & 29.9 & 15.4 & 9.8 & 18.6 & -14.0 \\
\hline Wool & -18.4 & 5.4 & 5.1 & 20.6 & -4.8 & -42.8 \\
\hline Meat & -2.3 & 41.3 & 2.4 & 33.0 & 26.9 & 6.0 \\
\hline Nontraditional exports & 2.1 & 11.4 & 7.8 & 15.6 & 11.9 & 8.4 \\
\hline
\end{tabular}

Sources: Central Bank of Uruguay; and Fund staff estimates.

1/ Starting in 1993, a new merchandise classification scheme is in place which may affect comparability with data from previous years. 
Table 46. Uruguay: Merchandise Exports, f.o.b.

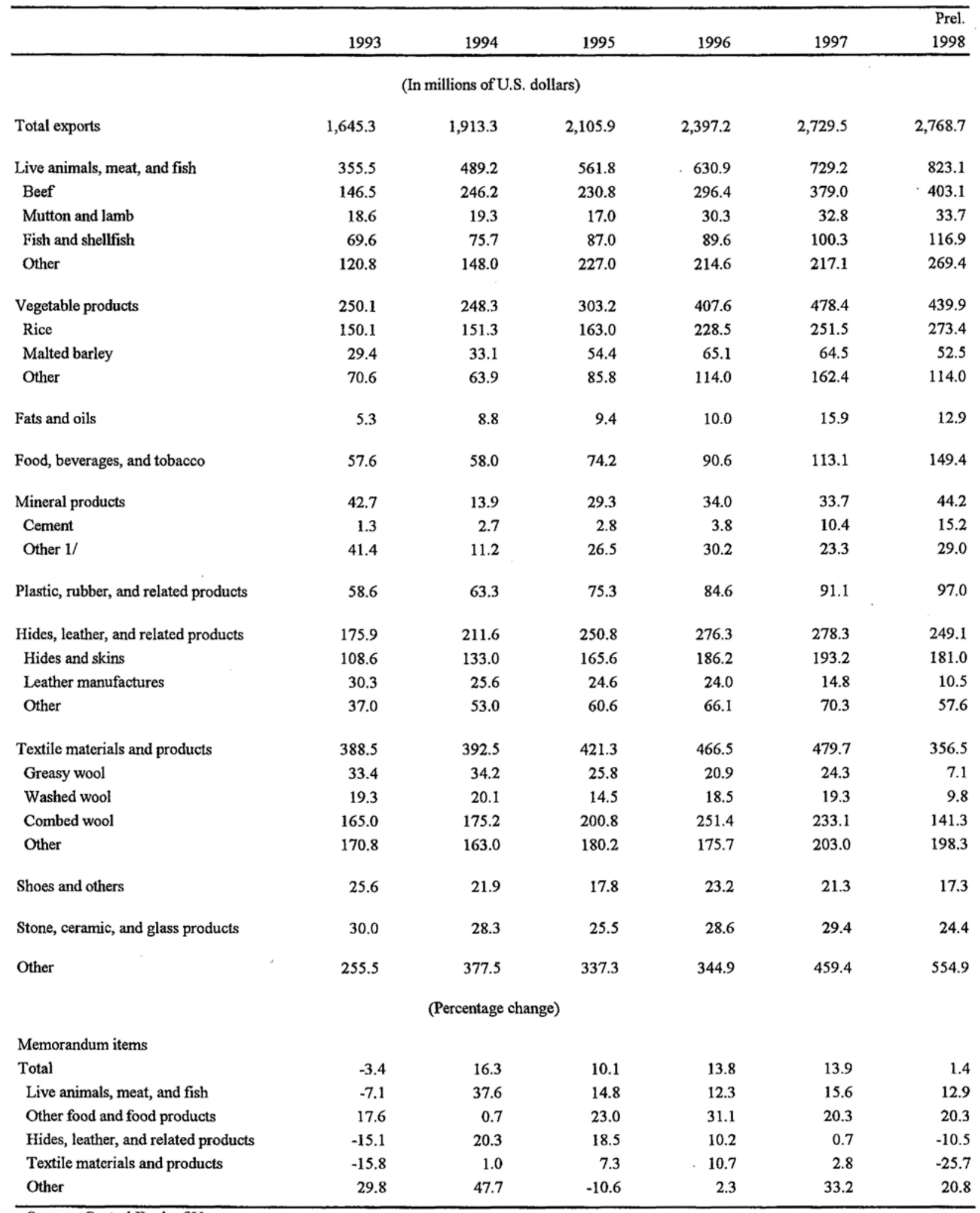

Source: Central Bank of Uruguay.

1/ Includes exports of electricity to Argentina. 
Table 47. Uruguay: Summary of Merchandise Imports, c.i.f.

\begin{tabular}{|c|c|c|c|c|c|c|}
\hline & 1993 & 1994 & 1995 & 1996 & 1997 & $\begin{array}{l}\text { Prel. } \\
1998 \\
\end{array}$ \\
\hline \multicolumn{7}{|c|}{ (In millions of U.S. dollars) } \\
\hline Total imports & $2,325.7$ & $2,786.1$ & $2,866.9$ & $3,322.8$ & $3,716.0$ & $3,808.2$ \\
\hline Consumer goods & 642.9 & 763.3 & 811.8 & 864.9 & $1,038.7$ & $1,091.0$ \\
\hline Intermediate goods & $1,283.0$ & $1,590.1$ & $1,567.6$ & $1,856.7$ & $1,991.9$ & $1,995.0$ \\
\hline Petroleum and derivatives & 193.1 & 237.0 & 271.6 & 337.6 & 318.3 & 205.0 \\
\hline Other & $1,089.9$ & $1,353.1$ & $1,296.0$ & $1,519.1$ & $1,673.6$ & $1,790.0$ \\
\hline Capital goods & 399.8 & 432.7 & 487.5 & 601.2 & 685.4 & 722.2 \\
\hline \multicolumn{7}{|c|}{ (In percent of total) } \\
\hline $\begin{array}{l}\text { Total } \\
\text { Consumer goods } \\
\text { Intermediate goods } \\
\text { Capital goods }\end{array}$ & $\begin{array}{r}100.0 \\
27.6 \\
55.2 \\
17.2\end{array}$ & $\begin{array}{r}100.0 \\
27.4 \\
57.1 \\
15.5\end{array}$ & $\begin{array}{r}100.0 \\
28.3 \\
54.7 \\
17.0\end{array}$ & $\begin{array}{r}100.0 \\
26.0 \\
55.9 \\
18.1\end{array}$ & $\begin{array}{r}100.0 \\
28.0 \\
53.6 \\
18.4\end{array}$ & $\begin{array}{r}100.0 \\
28.6 \\
52.4 \\
19.0\end{array}$ \\
\hline \multicolumn{7}{|c|}{ (Percentage change) } \\
\hline \multicolumn{7}{|l|}{ Memorandum items } \\
\hline Imports & 13.7 & 19.8 & 2.9 & 15.9 & 11.8 & 2.5 \\
\hline Consumer goods & 41.9 & 18.7 & 6.4 & 6.5 & 20.1 & 5.0 \\
\hline Intermediate goods & 2.4 & 23.9 & -1.4 & 18.4 & 7.3 & 0.2 \\
\hline Petroleum and derivatives & -13.3 & 22.7 & 14.6 & 24.3 & -5.7 & -35.6 \\
\hline Other & 5.8 & 24.1 & -4.2 & 17.2 & 10.2 & 7.0 \\
\hline Capital goods & 18.0 & 8.2 & 12.7 & 23.3 & 14.0 & 5.4 \\
\hline Oil imports & -13.3 & 22.7 & 14.6 & 24.3 & -5.7 & -35.6 \\
\hline Non-oil imports & 17.0 & 19.5 & 1.8 & 15.0 & 13.8 & 6.0 \\
\hline Total imports as percent of GDP & 17.2 & 17.1 & 15.9 & 17.4 & 18.6 & 18.3 \\
\hline
\end{tabular}

Sources: Central Bank of Uruguay; and Fund staff estimates. 
Table 48. Uruguay: Merchandise Imports, c.i.f.

(In millions of U.S. dollars)

\begin{tabular}{|c|c|c|c|c|c|c|}
\hline & 1993 & 1994 & 1995 & 1996 & 1997 & $\begin{array}{l}\text { Prel. } \\
1998 \\
\end{array}$ \\
\hline Total imports, c.i.f. & $2,325.7$ & $2,786.1$ & $2,866.9$ & $3,322.8$ & $3,716.0$ & $3,808.2$ \\
\hline Vegetable products & 88.1 & 119.2 & 105.2 & 126.8 & 119 & 118.5 \\
\hline Fats and oils & 13.7 & 19.9 & 19.6 & 21 & 22.3 & 24 \\
\hline Food, beverages and tobacco & 97.8 & 150.4 & 171.1 & 213.1 & 239.2 & 256.5 \\
\hline Mineral products, including fuels & 225.9 & 283.5 & 306.6 & 382.4 & 357.9 & 240 \\
\hline Petroleum & 18.5 & 22.8 & 177.3 & 225.2 & 203.1 & 163.4 \\
\hline Other & 207.4 & 260.7 & 129.3 & 157.2 & 154.8 & 76.6 \\
\hline Chemicals and related products & 268.1 & 319.7 & 340.1 & 452.1 & 491.8 & 512.4 \\
\hline Plastics, resins, and rubber & 142.5 & 165.5 & 201.1 & 217.1 & 254.2 & 257.9 \\
\hline Hides, leather, and related products & 28.1 & 35.9 & 40.3 & 56.5 & 44.3 & 50.6 \\
\hline Paper, cellulose, and printed matter & 60.1 & 74.7 & 97.1 & 111.6 & 128.5 & 144.8 \\
\hline Textile materials and products & 125.9 & 167.7 & 194.6 & 192.8 & 215.3 & 207 \\
\hline Stone, ceramic, and glass products & 37.3 & 44.3 & 49.7 & 48.9 & 59.4 & 65.8 \\
\hline Metals and metal products & 132.2 & 146.9 & 153.3 & 192.7 & 194.5 & 226.8 \\
\hline Machinery and equipment & 543.1 & 585.6 & 599.5 & 707.9 & 841.9 & 852.8 \\
\hline Transport equipment & 407 & 428.8 & 387.8 & 373.2 & 471.6 & 553.4 \\
\hline Precision instruments & 52.1 & 92.1 & 64.7 & 72.2 & 84.2 & 89.1 \\
\hline Miscellaneous goods & 103.8 & 151.9 & 136.2 & 154.5 & 191.9 & 208.6 \\
\hline
\end{tabular}

Source: Central Bank of Uruguay. 
Table 49. Uruguay: Direction of Merchandise Trade

(In percent)

\begin{tabular}{|c|c|c|c|c|c|c|}
\hline & 1993 & 1994 & 1995 & 1996 & 1997 & $\begin{array}{r}\text { Prel. } \\
1998 \\
\end{array}$ \\
\hline \multicolumn{7}{|c|}{ I. Exports } \\
\hline Total & 99.9 & 100.1 & 100.0 & 100.0 & 100.0 & 100.0 \\
\hline Western Hemisphere & 62.0 & 62.0 & 60.2 & 61.9 & 63.2 & 69.7 \\
\hline ALADI & 51.7 & 53.7 & 53.0 & 53.7 & 55.6 & 62.4 \\
\hline Argentina & 19.2 & 20.0 & 12.7 & 11.3 & 13.0 & 18.5 \\
\hline Brazil & 22.3 & 25.7 & 33.2 & 34.7 & 34.5 & 33.8 \\
\hline Others & 10.2 & 8.0 & 7.1 & 7.7 & 8.1 & 10.1 \\
\hline United States & 9.0 & 6.8 & 5.8 & 7.0 & 5.9 & 5.7 \\
\hline Rest of Western Hemisphere & 1.3 & 1.5 & 1.4 & 1.2 & 1.7 & 1.6 \\
\hline Europe & 22.2 & 22.3 & 22.7 & 21.4 & 21.1 & 17.9 \\
\hline $\mathrm{EC}$ & 20.3 & 20.0 & 20.8 & 19.5 & 18.9 & 16.4 \\
\hline Germany & 6.4 & 6.3 & 5.6 & 4.7 & 4.4 & 4.0 \\
\hline Italy & 3.0 & 3.2 & 3.1 & 3.5 & 3.3 & 2.8 \\
\hline United Kingdom & 3.9 & 3.8 & 4.1 & 3.6 & 4.3 & 3.4 \\
\hline Others & 7.0 & 6.7 & 8.0 & 7.7 & 6.9 & 6.2 \\
\hline Rest of Europe & 1.9 & 2.3 & 1.9 & 1.9 & 2.2 & 1.5 \\
\hline Asia & 10.0 & 10.4 & 11.8 & 11.3 & 10.1 & 6.9 \\
\hline Japan & 0.8 & 1.1 & 0.9 & 1.0 & 1.1 & 0.8 \\
\hline Rest of Asia & 9.2 & 9.3 & 10.9 & 10.3 & 9.0 & 6.1 \\
\hline Middle East & 4.2 & 4.1 & 3.7 & 3.9 & 4.0 & 3.9 \\
\hline Africa & 0.3 & 0.3 & 0.7 & 0.8 & 0.8 & 1.0 \\
\hline Other countries & 1.2 & 1.0 & 0.9 & 0.7 & 0.8 & 0.6 \\
\hline \multicolumn{7}{|c|}{ II. Imports } \\
\hline Total & 100.2 & 100.0 & 100.0 & 100.1 & 100.1 & 100.0 \\
\hline Western Hemisphere & 65.4 & 64.3 & 62.9 & 63.8 & 62.9 & 61.7 \\
\hline ALADI & 52.9 & 52.9 & 51.3 & 50.1 & 49.8 & 48.5 \\
\hline Argentina & 20.6 & 23.4 & 21.2 & 20.8 & 21.3 & 22.0 \\
\hline Brazil & 27.6 & 25.5 & 24.4 & 22.4 & 21.6 & 20.9 \\
\hline Others & 4.7 & 4.0 & 5.7 & 6.9 & 6.9 & 5.6 \\
\hline United States & 9.6 & 9.3 & 9.8 & 12.0 & 11.6 & 12.1 \\
\hline Rest of Western Hemisphere & 2.9 & 2.1 & 1.8 & 1.7 & 1.5 & 1.1 \\
\hline Europe & 20.6 & 23.3 & 22.9 & 21.5 & 22.1 & 23.3 \\
\hline EC & 17.6 & 19.6 & 20.8 & 19.4 & 19.4 & 20.6 \\
\hline Germany & 3.9 & 3.6 & 3.6 & 3.0 & 3.2 & 3.3 \\
\hline Italy & 4.2 & 4.8 & 5.2 & 5.2 & 4.6 & 4.6 \\
\hline United Kingdom & 1.8 & 1.5 & 1.4 & 1.6 & 1.8 & 1.5 \\
\hline Others & 7.7 & 9.7 & 10.6 & 9.6 & 9.8 & 11.2 \\
\hline Rest of Europe & 3.0 & 3.7 & 2.1 & 2.1 & 2.7 & 2.7 \\
\hline Asia & 12.1 & 10.0 & 9.6 & 9.2 & 10.3 & 11.0 \\
\hline Japan & 5.2 & 2.8 & 2.6 & 2.2 & 2.6 & 2.4 \\
\hline Rest of Asia & 6.9 & 7.2 & 7.0 & 7.0 & 7.7 & 8.6 \\
\hline Middle East & 1.1 & 1.3 & 2.4 & 2.3 & 2.8 & 0.8 \\
\hline Africa & 0.5 & 0.5 & 1.4 & 2.6 & 1.0 & 2.3 \\
\hline Other countries & 0.5 & 0.6 & 0.8 & 0.7 & 1.0 & 0.9 \\
\hline
\end{tabular}


Table 50. Uruguay: Summary of External Debt

\begin{tabular}{|c|c|c|c|c|c|c|}
\hline & 1993 & 1994 & 1995 & 1996 & 1997 & $\begin{array}{l}\text { Prel. } \\
1998\end{array}$ \\
\hline \multicolumn{7}{|c|}{ (In millions of U.S. dollars) } \\
\hline Medium and long-term debt & $3,821.1$ & $4,562.6$ & $4,696.4$ & $4,937.4$ & $5,225.6$ & $5,647.0$ \\
\hline Public sector $1 /$ & $3,458.1$ & $4,143.3$ & $4,274.9$ & $4,540.6$ & $4,760.7$ & $5,161.1$ \\
\hline Private sector $2 /$ & 363.0 & 419.3 & 421.5 & 396.8 & 464.9 & 485.9 \\
\hline Short-term debt & $1,682.2$ & $1,366.5$ & $1,593.0$ & $1,814.3$ & $1,976.5$ & $2,553.0$ \\
\hline Public sector & 749.7 & 419.4 & 525.1 & 420.5 & 292.5 & 402.1 \\
\hline Treasury notes & 441.4 & 419.4 & 525.1 & 420.5 & 292.5 & 402.1 \\
\hline Private sector & 932.5 & 947.1 & $1,067.9$ & $1,393.8$ & $1,684.0$ & $2,150.9$ \\
\hline Commercial banks & 932.5 & 947.1 & $1,067.9$ & $1,393.8$ & $1,684.0$ & $2,150.9$ \\
\hline Total external debt & $5,503.3$ & $5,929.1$ & $6,289.4$ & $6,751.7$ & $7,202.1$ & $8,200.0$ \\
\hline Public & $4,207.8$ & $4,562.7$ & $4,800.0$ & $4,961.1$ & $5,053.2$ & $5,563.2$ \\
\hline Private & $1,295.5$ & $1,366.4$ & $1,489.4$ & $1,790.6$ & $2,148.9$ & $2,636.8$ \\
\hline \multicolumn{7}{|c|}{ (In percent) } \\
\hline Ratio of total debt to GDP & 40.7 & 36.4 & 34.9 & 35.3 & 36.1 & 39.4 \\
\hline Ratio of public debt to GDP & 31.1 & 28.0 & 26.6 & 25.9 & 25.3 & 26.7 \\
\hline $\begin{array}{l}\text { Ratio of total debt to exports of goods } \\
\text { and nonfactor services }\end{array}$ & 206.9 & 182.5 & 179.4 & 175.5 & 170.5 & 194.1 \\
\hline
\end{tabular}

Sources: Central Bank of Uruguay; and Fund staff estimates.

1/ Includes long-term debt of the central bank.

2/ Suppliers' credits (including short-term). 
Table 51. Uruguay: Summary of Public External Debt Service

\begin{tabular}{|c|c|c|c|c|c|c|}
\hline & 1993 & 1994 & 1995 & 1996 & 1997 & $\begin{array}{l}\text { Prel. } \\
1998 \\
\end{array}$ \\
\hline \multicolumn{7}{|c|}{ (In millions of U.S. dollars) } \\
\hline Debt service payments $1 /$ & $1,128.4$ & 987.8 & $1,082.2$ & $1,078.5$ & 695.7 & 595.8 \\
\hline Amortization & 830.1 & 673.6 & 727.3 & 749.3 & 345.0 & 221.7 \\
\hline Medium- and long-term $2 /$ & 270.6 & 232.2 & 307.9 & 227.0 & 220.0 & 221.7 \\
\hline Short-term 3/ & 559.5 & 441.4 & 419.4 & 522.3 & 125.0 & 0.0 \\
\hline Interest & 298.3 & 314.2 & 354.9 & 329.2 & 350.7 & 374.1 \\
\hline Medium- and long-term & 269.5 & 284.8 & 317.2 & 284.2 & 305.4 & 335.4 \\
\hline Short-term & 28.8 & 29.4 & 37.7 & 45 & 45.3 & 38.7 \\
\hline \multicolumn{7}{|l|}{ Debt service payments excluding } \\
\hline \multicolumn{7}{|l|}{ Memorandum items: } \\
\hline IMF repurchases & 14.3 & 10.8 & 9.7 & 11.6 & 8.7 & 0.0 \\
\hline IMF charges & 6.9 & 4.7 & 4.2 & 3.3 & 0.9 & 0.0 \\
\hline \multicolumn{7}{|c|}{ (In percent) } \\
\hline \multicolumn{7}{|l|}{ Debt service ratios $4 /$} \\
\hline Public debt service including the Fund & 42.4 & 30.4 & 30.9 & 28.0 & 16.5 & 14.1 \\
\hline Interest & 11.2 & 9.7 & 10.1 & 8.6 & 8.3 & 8.9 \\
\hline Amortization & 31.2 & 20.7 & 20.7 & 19.5 & 8.2 & 5.2 \\
\hline Public debt service excluding the Fund & 41.6 & 29.9 & 30.5 & 27.6 & 16.2 & 14.1 \\
\hline Interest & 11.0 & 9.5 & 10.0 & 8.5 & 8.3 & 8.9 \\
\hline Amortization & 30.7 & 20.4 & 20.5 & 19.2 & 8.0 & 5.2 \\
\hline
\end{tabular}

Sources: Central Bank of Uruguay; and Fund staff estimates.

1/ After rescheduling. Includes payments to the Fund.

2/ 1991 figure includes US\$461 million related to the debt buy-back and interest and principal collateral components of the debt and debt service reduction operation with commercial banks of February 1991.

3/ All short-term debt is assumed to be "rolled over" once a year.

4/ In relation to exports of goods and nonfactor services; includes amortization of treasury bonds and notes denominated in foreign currency in hands of nonresidents, and medium-term suppliers' credits. Debt service ratios in 1991 include the cost of the debt reduciton operation. Excludes short-term debt rollover. 
Table 52. Uruguay: Disbursements, Amortization, and Outstanding External Public Debt

(In millions of U.S. dollars)

\begin{tabular}{|c|c|c|c|c|}
\hline & \multicolumn{3}{|c|}{ Transactions during 1995} & \multirow{2}{*}{$\begin{array}{r}1995 \\
\text { Outstanding } \\
\end{array}$} \\
\hline & Disbursements & Amortization & $\overline{\text { Adjustment } 1 /}$ & \\
\hline Medium- and long-term debt & 397.0 & 259.9 & -5.5 & $4,274.9$ \\
\hline Central Government 2/ & 121.4 & 84.0 & 8.0 & $1,331.4$ \\
\hline University of Labor (UTU) & 2.3 & 0.3 & 0.0 & 2.3 \\
\hline Treasury & 14.7 & 42.7 & 6.8 & 845.6 \\
\hline Ministry of Agriculture and Fishing (MAP) & 9.1 & 7.4 & -0.9 & 95.6 \\
\hline Ministry of Industry and Energy & 0.0 & 0.0 & 0.0 & 0.0 \\
\hline Commission of Agricultural Planning & 0.0 & 0.9 & -0.6 & 0.0 \\
\hline Ministry Public Works and Transportation & 33.7 & 29.5 & 2.6 & 252.3 \\
\hline Ministry of Education and Culture & 7.6 & 0.0 & 0.0 & 12.7 \\
\hline University of Montevideo & 0.2 & 0.7 & 0.0 & 2.5 \\
\hline Air Force & 0.0 & 0.0 & 0.0 & 0.0 \\
\hline Ministry of Public Health (MSP) & 3.8 & 0.9 & -0.1 & 34.4 \\
\hline National Public Education Adm. (ANEP) & 11.3 & 0.0 & 0.0 & 28.2 \\
\hline Official Broadcasting Services (SODRE) & 0.3 & 0.4 & 0.0 & 5.3 \\
\hline Customs & 0.0 & 0.0 & 0.0 & 0.0 \\
\hline Ministry of Housing & 32.0 & 0.0 & 0.1 & 32.6 \\
\hline Ministry of Defense & 6.4 & 1.2 & 0.1 & 19.9 \\
\hline Local governments & 10.4 & 4.7 & 2.4 & 71.8 \\
\hline Official banks & 1.2 & 3.3 & -1.4 & 204.4 \\
\hline BROU & 1.2 & 3.3 & -0.2 & 73.7 \\
\hline $\mathrm{BHU}$ & 0.0 & 0.0 & -1.2 & 130.7 \\
\hline Monetary authorities 3 / & 0.0 & 7.2 & 4.8 & 101.0 \\
\hline Public enterprises & 23.5 & 57.9 & -19.3 & $1,002.8$ \\
\hline UTE & 12.8 & 42.8 & -18.4 & 754.8 \\
\hline ANTEL & 9.0 & 6.6 & -0.1 & 87.1 \\
\hline ANCAP & 0.7 & 2.0 & 0.0 & 21.1 \\
\hline OSE & 0.0 & 2.8 & 1.0 & 22.5 \\
\hline ANP & 0.0 & 2.3 & 0.1 & 0.0 \\
\hline Salto Grande & 0.0 & 0.0 & -2.1 & 69.0 \\
\hline PLUNA & 0.0 & 1.4 & 1.1 & 23.8 \\
\hline AFE & 1.0 & 0.0 & -0.9 & 24.5 \\
\hline Treasury bonds & 240.5 & 59.9 & 0.0 & $1,429.8$ \\
\hline Suppliers' credits & 0.0 & 42.9 & 0.0 & 133.7 \\
\hline \multicolumn{5}{|l|}{ Memorandum items } \\
\hline Treasury notes & 105.7 & 0.0 & 0.0 & 525.1 \\
\hline BROU, net nonresident deposits & 43.4 & 0.0 & 0.0 & 326.4 \\
\hline BHU, net nonresident deposits & 0.0 & 3.1 & 0.0 & 25.9 \\
\hline \multicolumn{5}{|l|}{ Total, including treasury notes, } \\
\hline $\mathrm{BROU}$ and $\mathrm{BHU}$ & 546.1 & 263.0 & -5.5 & $5,152.3$ \\
\hline
\end{tabular}


$-65-$

Table 52. Uruguay: Disbursements, Amortization, and Outstanding External Public Debt

(In millions of U.S. dollars)

\begin{tabular}{|c|c|c|c|c|}
\hline & \multicolumn{3}{|c|}{ Transactions during 1996} & \multirow{2}{*}{$\begin{array}{r}1996 \\
\text { Outstanding } \\
\end{array}$} \\
\hline & Disbursements & Amortization & $\overline{\text { Adjustment } 1 /}$ & \\
\hline Medium- and long-term debt & 503.6 & 227.6 & -10.3 & $4,540.6$ \\
\hline Central Government 2/ & 139.6 & 84.6 & -12.5 & $1,373.9$ \\
\hline University of Labor (UTU) & 1.3 & 0.0 & -0.6 & 3.0 \\
\hline Treasury & 70.7 & 40.8 & 0.8 & 876.3 \\
\hline Ministry of Agriculture and Fishing (MAP) & 7.0 & 7.4 & 2.0 & 97.2 \\
\hline Ministry of Industry and Energy & 0.0 & 0.0 & 0.0 & 0.0 \\
\hline Commission of Agricultural Planning & 0.0 & 0.0 & 0.0 & 0.0 \\
\hline Ministry Public Works and Transportation & 45.7 & 34.6 & -16.2 & 247.2 \\
\hline Ministry of Education and Culture & 3.8 & 0.0 & 3.6 & 20.1 \\
\hline University of Montevideo & 0.0 & 0.4 & -0.4 & 1.7 \\
\hline Air Force & 0.0 & 0.0 & 0.0 & 0.0 \\
\hline Ministry of Public Health (MSP) & 0.5 & 0.0 & 0.1 & 35.0 \\
\hline National Public Education Adm. (ANEP) & 3.6 & 0.0 & 0.0 & 31.8 \\
\hline Official Broadcasting Services (SODRE) & 0.2 & 0.4 & -0.1 & 5.0 \\
\hline Customs & 0.6 & 0.0 & 0.2 & 0.8 \\
\hline Ministry of Housing & 3.3 & 0.0 & -0.2 & 35.7 \\
\hline Ministry of Defense & 2.9 & 1.0 & -1.7 & 20.1 \\
\hline Local governments & 2.5 & 4.4 & -3.9 & 66.0 \\
\hline Official banks & 1.0 & 3.5 & 1.8 & 203.7 \\
\hline BROU & 1.0 & 3.5 & 0.4 & 71.6 \\
\hline $\mathrm{BHU}$ & 0.0 & 0.0 & 1.4 & 132.1 \\
\hline Monetary authorities 3/ & 0.0 & 6.7 & 7.8 & 102.1 \\
\hline Public enterprises & 41.3 & 83.0 & -3.2 & 957.9 \\
\hline UTE & 13.4 & 69.5 & -2.3 & 696.4 \\
\hline ANTEL & 5.8 & 6.0 & 0.2 & 87.1 \\
\hline ANCAP & 0.0 & 2.0 & 0.1 & 19.2 \\
\hline OSE & 21.9 & 2.7 & -0.2 & 41.5 \\
\hline ANP & 0.0 & 0.0 & 0.2 & 0.2 \\
\hline Salto Grande & 0.0 & 0.0 & -0.8 & 68.2 \\
\hline PLUNA & 0.0 & 1.4 & 1.7 & 24.1 \\
\hline AFE & 0.2 & 1.4 & -2.1 & 21.2 \\
\hline Treasury bonds & 297.4 & 12.7 & 1.7 & $1,716.2$ \\
\hline Suppliers' credits & 21.8 & 32.7 & -2.0 & 120.8 \\
\hline \multicolumn{5}{|l|}{ Memorandum items } \\
\hline Treasury notes & 25.5 & 130.1 & 0.0 & 420.5 \\
\hline BROU, net nonresident deposits & 36.1 & 0.0 & 0.1 & 362.6 \\
\hline BHU, net nonresident deposits & 4.5 & 0.6 & 0.0 & 29.8 \\
\hline \multicolumn{5}{|l|}{ Total, including treasury notes, } \\
\hline & 569.7 & 358.3 & -10.2 & $5,353.5$ \\
\hline
\end{tabular}




$$
-66-
$$

Table 52. Uruguay: Disbursements, Amortization, and Outstanding External Public Debt

(In millions of U.S. dollars)

\begin{tabular}{|c|c|c|c|c|}
\hline & \multicolumn{3}{|c|}{ Transactions during 1997} & \multirow{2}{*}{$\begin{array}{r}1997 \\
\text { Outstanding } \\
\end{array}$} \\
\hline & Disbursements & Amortization & Adjustment $1 /$ & \\
\hline Medium- and long-term debt & 982.2 & 715.6 & -46.5 & $4,760.7$ \\
\hline Central Government $2 /$ & 247.5 & 382.4 & 13.2 & $1,252.2$ \\
\hline University of Labor (UTU) & 3.4 & 0.0 & 0.0 & 6.4 \\
\hline Treasury & 165.8 & 339.7 & 28.8 & 731.2 \\
\hline Ministry of Agriculture and Fishing (MAP) & 10.4 & 9.9 & -1.7 & 96.0 \\
\hline Ministry of Industry and Energy & 0.0 & 0.0 & 0.0 & 0.0 \\
\hline Commission of Agricultural Planning & 0.0 & 0.0 & 0.0 & 0.0 \\
\hline Ministry Public Works and Transportation & 45.7 & 30.1 & -13.1 & 249.7 \\
\hline Ministry of Education and Culture & 6.8 & 0.0 & -0.4 & 26.5 \\
\hline University of Montevideo & 0.0 & 0.7 & 0.1 & 1.1 \\
\hline Air Force & 0.4 & 0.0 & -0.4 & 0.0 \\
\hline Ministry of Public Health (MSP) & 0.8 & 0.0 & 0.0 & 35.8 \\
\hline National Public Education Adm. (ANEP) & 6.7 & 0.0 & 0.0 & 38.5 \\
\hline Official Broadcasting Services (SODRE) & 0.0 & 0.4 & 0.0 & 4.6 \\
\hline Customs & 0.2 & 0.0 & 0.0 & 1.0 \\
\hline Ministry of Housing & 7.3 & 0.0 & -0.1 & 42.9 \\
\hline Ministry of Defense & 0.0 & 1.6 & 0.0 & 18.5 \\
\hline Local governments & 0.0 & 5.2 & -3.1 & 57.7 \\
\hline Official banks & 1.2 & 14.6 & -3.9 & 186.4 \\
\hline BROU & 1.2 & 3.5 & -3.9 & 65.4 \\
\hline BHU & 0.0 & 11.1 & 0.0 & 121.0 \\
\hline Monetary authorities 3/ & 296.5 & 0.1 & -0.5 & 398.0 \\
\hline Public enterprises & 97.5 & 74.4 & -52.2 & 928.8 \\
\hline UTE & 53.4 & 57.7 & -28.8 & 663.3 \\
\hline ANTEL & 0.0 & 4.1 & -2.5 & 80.5 \\
\hline ANCAP & 0.0 & 2.0 & -0.6 & 16.6 \\
\hline OSE & 37.1 & 2.5 & -9.8 & 66.3 \\
\hline ANP & 5.8 & 0.0 & -5.7 & 0.3 \\
\hline Salto Grande & 0.0 & 3.7 & -2.0 & 62.5 \\
\hline PLUNA & 0.0 & 2.0 & 0.0 & 22.1 \\
\hline AFE & 1.2 & 2.4 & -2.8 & 17.2 \\
\hline Treasury bonds & 339.5 & 196.6 & 0.0 & $1,859.1$ \\
\hline Suppliers' credits & 0.0 & 42.3 & 0.0 & 78.5 \\
\hline \multicolumn{5}{|l|}{ Memorandum items } \\
\hline Treasury notes & 0.0 & 128.0 & 0.0 & 292.5 \\
\hline BROU, net nonresident deposits & 14.9 & 0.0 & 0.0 & 377.5 \\
\hline BHU, net nonresident deposits & 0.0 & 1.5 & 0.0 & 28.3 \\
\hline $\begin{array}{l}\text { Total, including treasury notes, } \\
\text { BROU and BHU }\end{array}$ & 997.1 & 845.1 & -46.5 & $5,459.0$ \\
\hline
\end{tabular}


Table 52. Uruguay: Disbursements, Amortization, and Outstanding External Public Debt

(In millions of U.S. dollars)

\begin{tabular}{|c|c|c|c|c|}
\hline & \multicolumn{3}{|c|}{ Transactions during 1998} & \multirow{2}{*}{$\begin{array}{r}1998 \\
\text { Outstanding }\end{array}$} \\
\hline & Disbursements & Amortization & Adjustment 1/ & \\
\hline Medium- and long-term debt & 586.8 & 238.6 & 52.2 & $5,161.1$ \\
\hline Central Government $2 /$ & 240.9 & 114.7 & 50.6 & $1,429.0$ \\
\hline University of Labor (UTU) & 5.0 & 0.0 & -0.1 & 11.3 \\
\hline Treasury & 163.0 & 69.1 & 48.0 & 873.1 \\
\hline Ministry of Agriculture and Fishing (MAP) & 10.7 & 9.9 & 0.6 & 97.4 \\
\hline Ministry of Industry and Energy & 0.0 & 0.0 & 0.0 & 0.0 \\
\hline Commission of Agricultural Planning & 0.0 & 0.0 & 0.0 & 0.0 \\
\hline Ministry Public Works and Transportation & 49.2 & 33.0 & 2.3 & 268.2 \\
\hline Ministry of Education and Culture & 1.0 & 0.0 & 0.4 & 27.9 \\
\hline University of Montevideo & 0.0 & 0.7 & 0.0 & 0.4 \\
\hline Air Force & 0.8 & 0.0 & 0.3 & 1.1 \\
\hline Ministry of Public Health (MSP) & 3.7 & 0.0 & -0.4 & 39.1 \\
\hline National Public Education Adm. (ANEP) & 5.2 & 0.0 & -0.4 & 43.3 \\
\hline Official Broadcasting Services (SODRE) & 0.0 & 0.4 & -0.1 & 4.1 \\
\hline Customs & 0.0 & 0.0 & 0.0 & 1.0 \\
\hline Ministry of Housing & 1.9 & 0.0 & 0.0 & 44.8 \\
\hline Ministry of Defense & 0.4 & 1.6 & 0.0 & 17.3 \\
\hline Local governments & 0.0 & 5.0 & 2.0 & 54.7 \\
\hline Official banks & 2.0 & 12.0 & 0.0 & 176.4 \\
\hline BROU & 1.7 & 5.3 & 0.3 & 62.1 \\
\hline BHU & 0.3 & 6.7 & -0.3 & 114.3 \\
\hline Monetary authorities 3/ & 0.0 & 16.6 & -14.1 & 367.3 \\
\hline Public enterprises & 32.7 & 90.3 & 13.7 & 884.9 \\
\hline UTE & 27.2 & 67.9 & 2.6 & 625.2 \\
\hline ANTEL & 0.0 & 5.4 & -0.2 & 74.9 \\
\hline ANCAP & 0.0 & 2.4 & -0.1 & 14.1 \\
\hline OSE & 5.3 & 9.2 & 8.9 & 71.3 \\
\hline ANP & 0.2 & 0.0 & -0.2 & 0.3 \\
\hline Salto Grande & 0.0 & 3.2 & -0.2 & 59.1 \\
\hline PLUNA & 0.0 & 1.1 & -0.2 & 20.8 \\
\hline AFE & 0.0 & 1.1 & 3.1 & 19.2 \\
\hline Treasury bonds & 305.1 & 0.0 & 0.0 & $2,164.2$ \\
\hline Suppliers' credits & 6.1 & 0.0 & 0.0 & 84.6 \\
\hline \multicolumn{5}{|l|}{ Memorandum items } \\
\hline Treasury notes & 109.6 & 0.0 & 0.0 & 402.1 \\
\hline BROU, net nonresident deposits & 0.0 & 13.3 & 0.0 & 364.2 \\
\hline BHU, net nonresident deposits & 0.0 & 0.5 & 0.0 & 27.8 \\
\hline $\begin{array}{l}\text { Total, inchuding treasury notes, } \\
\text { BROU and BHU }\end{array}$ & 696.4 & 252.4 & 52.2 & $5,955.2$ \\
\hline
\end{tabular}

Sources: Central Bank of Uruguay; and Fund staff estimates.

1/ Reflects valuation of adjustments due to changes in exchange rates.

2/ Excludes treasury bills and bonds.

3/ Excludes use of IMF credit. 
Table 53. Uruguay: Amortization for Medium- and Long-Term Public Sector External Debt

(In millions of U.S. dollars)

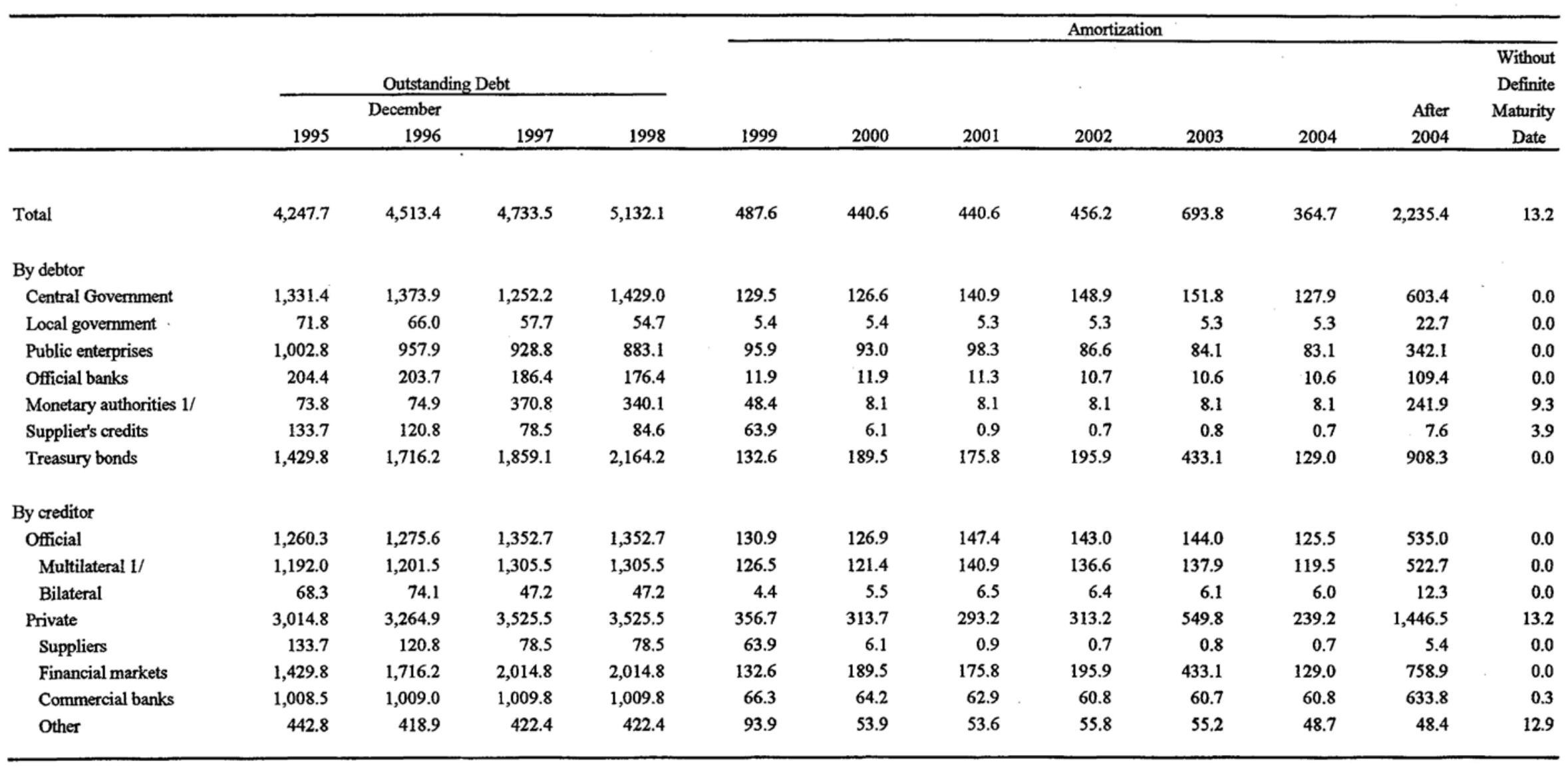

Source: Central Bank of Uruguay.

1/ Includes IMF credit. 
Table 54. Uruguay: Net International Reserves of the Central Bank

(In millions of U.S. dollars, unless otherwise indicated)

\begin{tabular}{|c|c|c|c|c|c|c|}
\hline & 1993 & 1994 & 1995 & 1996 & 1997 & 1998 \\
\hline Assets & $1,286.7$ & $1,516.1$ & $1,824.0$ & $1,908.9$ & $2,068.4$ & $2,589.3$ \\
\hline Gold 1/ & 496.2 & 522.0 & 663.8 & 642.1 & 509.7 & 513.4 \\
\hline SDRs & 0.0 & 0.0 & 4.0 & 3.9 & 0.0 & 0.7 \\
\hline Correspondents $2 /$ & 1.4 & 2.0 & 7.9 & 6.5 & 6.9 & 56.9 \\
\hline Other assets & 789.1 & 992.1 & $1,148.3$ & $1,256.4$ & $1,551.8$ & $2,018.3$ \\
\hline Liabilities & 84.9 & 86.6 & 56.1 & 33.7 & 2.5 & 162.2 \\
\hline $\mathrm{IMF}$ & 38.4 & 29.9 & 21.0 & 8.6 & 0.0 & 160.7 \\
\hline Other liabilities & 46.5 & 56.7 & 35.1 & 25.1 & 2.5 & 1.5 \\
\hline Net international reserves & $1,201.8$ & $1,429.5$ & $1,767.9$ & $1,875.2$ & $2,065.9$ & $2,427.1$ \\
\hline \multicolumn{7}{|l|}{ Memorandum items } \\
\hline Gold (millions of fine troy ounces) & $1,700.0$ & $1,704.0$ & $1,715.0$ & $1,736.3$ & $1,760.0$ & $1,783.6$ \\
\hline $\begin{array}{l}\text { Gross reserves (in months of } \\
\text { imports of goods and services) }\end{array}$ & 5.4 & 5.2 & 6.1 & 5.8 & 5.7 & 6.9 \\
\hline
\end{tabular}

Sources: Central Bank of Uruguay; IMF, International Financial Statistics; and Fund staff estimates.

1/ Price of gold per troy ounce: US\$307.71 in 1990, US\$283.04 in 1991, US\$266.80 in 1992, US\$291.89 in 1993 US\$306.36 in 1994, and US\$387.05 in 1995 and 1996.

2/ Overnight accounts. 
Table 55. Uruguay: Exchange Rates 1/

Period Average

End of Period

(New Uruguayan pesos per U.S. dollar)

1982

1983

1984

1985

1986

1987

1988

1989

1990

1991

1992

1993

1994

March

June

September

December

1995

March

June

September

December

1996

March

June

September

December

1997

March

June

September

December

1998

March

June

September

December

13.85

34.38

55.89

101.16

151.43

225.52

359.44

634.70

$1,235.10$

$2,088.58$

$3,107.82$

$4,134.00$

(Uruguayan pesos per U.S. dollar) 21

\begin{tabular}{ll}
4.56 & 4.69 \\
4.84 & 4.99 \\
5.25 & 5.57 \\
5.54 & 5.60 \\
& \\
5.84 & \\
6.14 & 5.95 \\
6.51 & 6.26 \\
6.92 & 6.64 \\
& 7.11 \\
7.34 & \\
7.74 & \\
8.24 & 7.54 \\
8.54 & 7.99 \\
& 8.36 \\
8.91 & 8.71 \\
9.30 & \\
9.66 & \\
9.90 & 9.08 \\
& 9.49 \\
10.05 & 9.75 \\
10.25 & 9.97 \\
10.54 & \\
10.62 & \\
& \\
& \\
& 10.21 \\
& 10.42 \\
& 10.83 \\
& 10.78 \\
\hline & \\
& \\
& \\
& \\
& \\
& \\
&
\end{tabular}

Source: Central Bank of Uruguay.

$1 /$ Buying rate. Until 1978 , the exchange rates listed are those prevailing in the commercial foreign exchange market. On October 17, 1978, the Central Bank unified the commercial and financial foreign exchange markets and on December 26, 1978, the Government announced that the new peso would be depreciated each month according to preannounced rate. On November 26 , 1982, a managed float system was implemented.

2/ The Uruguayan peso replaced the new Uruguayan peso at the rate of 1,000 to 1 on March 1, 1993.

(C)International Monetary Fund. Not for Redistribution 
Table 56. Uruguay: Measures of Competitiveness

\begin{tabular}{|c|c|c|c|c|c|}
\hline & \multirow{2}{*}{$\begin{array}{c}\text { Real Effective } \\
\text { Exchange Rate 1/ }\end{array}$} & \multirow{2}{*}{$\begin{array}{c}\text { Wage-based } \\
\text { Real Effective } \\
\text { Exchange Rate 2/ }\end{array}$} & \multicolumn{3}{|c|}{ Bilateral Real Effective Exchange Rate } \\
\hline & & & Argentina & Brazil & Unites States \\
\hline \multicolumn{6}{|c|}{ Index 1990=100 } \\
\hline \multicolumn{6}{|l|}{1993} \\
\hline First quarter & 133.6 & 150.0 & 84.1 & 166.0 & 131.7 \\
\hline Second quarter & 139.6 & 158.3 & 86.3 & 169.4 & 138.3 \\
\hline Third quarter & 142.9 & 169.7 & 90.5 & 172.2 & 146.1 \\
\hline Four quarter & 146.5 & 163.4 & 92.2 & 175.9 & 148.7 \\
\hline \multicolumn{6}{|l|}{1994} \\
\hline First quarter & 143.6 & 162.5 & 93.3 & 162.5 & 149.9 \\
\hline Second quarter & 148.5 & 174.0 & 95.4 & 175.3 & 153.6 \\
\hline Third quarter & 131.6 & 159.7 & 91.8 & 135.8 & 149.1 \\
\hline Four quarter & 142.5 & 156.0 & 100.7 & 139.4 & 163.8 \\
\hline \multicolumn{6}{|l|}{1995} \\
\hline First quarter & 146.4 & 164.9 & 101.8 & 142.5 & 165.8 \\
\hline Second quarter & 143.8 & 161.9 & 105.0 & 143.8 & 170.2 \\
\hline Third quarter & 149.9 & 172.3 & 106.4 & 149.0 & 172.4 \\
\hline Four quarter & 148.2 & 158.3 & 104.7 & 146.7 & 169.4 \\
\hline \multicolumn{6}{|l|}{1996} \\
\hline First quarter & 147.6 & 161.6 & 105.7 & 145.9 & 168.3 \\
\hline Second quarter & 146.5 & 165.9 & 106.0 & 143.3 & 167.3 \\
\hline Third quarter & 147.7 & 173.7 & 107.1 & 146.5 & 169.0 \\
\hline Four quarter & 147.5 & 157.7 & 106.9 & 146.9 & 167.5 \\
\hline \multicolumn{6}{|l|}{1997} \\
\hline First quarter & 152.5 & 168.2 & 106.7 & 146.5 & $\begin{array}{l}167.8 \\
168.9\end{array}$ \\
\hline $\begin{array}{l}\text { Second quarter } \\
\text { Third quarter }\end{array}$ & $\begin{array}{l}153.2 \\
154.8\end{array}$ & $\begin{array}{l}171.6 \\
185.1\end{array}$ & $\begin{array}{l}106.4 \\
107.3\end{array}$ & $\begin{array}{l}147.1 \\
146.1\end{array}$ & $\begin{array}{l}168.9 \\
170.2\end{array}$ \\
\hline Fourth quarter & 154.4 & 166.0 & 107.2 & 144.3 & 171.0 \\
\hline \multicolumn{6}{|l|}{1998} \\
\hline First quarter & 158.0 & 176.6 & 107.9 & 143.5 & 171.1 \\
\hline Second quarter & 157.4 & 180.0 & 108.2 & 142.7 & 172.4 \\
\hline Third quarter & 157.3 & 189.7 & 108.8 & 139.0 & 173.3 \\
\hline Fourth quarter & 153.7 & 166.1 & 107.2 & 134.7 & 172.3 \\
\hline \multicolumn{6}{|c|}{ (Average annual percentage change) } \\
\hline 1985 & -0.4 & 7.3 & 6.8 & -1.7 & -8.2 \\
\hline 1986 & 6.0 & 11.0 & -0.5 & 6.2 & 15.3 \\
\hline 1987 & 1.4 & 6.6 & 6.3 & -1.5 & 6.4 \\
\hline 1988 & -4.7 & -4.9 & 3.3 & -12.1 & -2.1 \\
\hline 1989 & 1.8 & 1.3 & 28.1 & -12.9 & 2.3 \\
\hline 1990 & -15.3 & -19.0 & -33.2 & -15.9 & 3.3 \\
\hline 1991 & 15.0 & 22.0 & -14.4 & 38.0 & 12.2 \\
\hline 1992 & 6.9 & 11.1 & -5.5 & 16.3 & 8.9 \\
\hline 1993 & 14.5 & 18.3 & 9.1 & 6.5 & 15.6 \\
\hline 1994 & 0.6 & 1.7 & 8.0 & -10.3 & 9.1 \\
\hline 1995 & 3.9 & 0.8 & 9.6 & -5.1 & 10.0 \\
\hline 1996 & 0.2 & 0.2 & 1.9 & 0.1 & -0.8 \\
\hline 1997 & 4.3 & 4.9 & 0.4 & 0.2 & 0.9 \\
\hline 1998 & 1.9 & 3.1 & 1.1 & -4.1 & 1.7 \\
\hline
\end{tabular}

Sources: Central Bank of Uruguay; IMF Information Notice System; and Fund staff estimates.

1 / Based on consumer prices. INS data with updated trade weights.

$2 /$ Real private sector wages multiplied by real effective exchange rate. Increase indicates loss in competitiveness. 


\section{UNEMPLOYMENT AND REAL WAGES ${ }^{1}$}

Uruguay's process of market-oriented reforms during the 1990s has boosted trade flows and productivity, and brought down inflation to a single digit. However, at more than 10 percent, the unemployment rate continues to be high by historical standards (Figure 1), despite almost a decade of impressive GDP growth. Also, Uruguayan (and Argentine) unemployment is high compared with other countries in the region (Figure 2) ${ }^{2}$ This note presents background facts to analyze the nature of Uruguayan unemployment and the role of union wage bargaining for the labor market outcome.

A Phillips-curve diagram is helpful to gain a first overview of recent developments on the labor market (Figure 3). In the early phase of stabilization, between January 1991 (when inflation peaked at 140 percent) and October 1993, the relationship between inflation and unemployment was almost vertical. Inflation could be reduced without much cost, as expectations adapted quickly. The "sacrifice ratio" was minimal since unemployment remained constant or even fell slightly. However, reducing inflation became more difficult as it declined below 50 percent. Residual inflation expectations were harder to overcome and structural reforms to make the economy more efficient produced layoffs. A (first) negatively sloped Phillips curve appears in late 1993, forcing unemployment up to 13 percent over the next three years. However, the government remained strongly committed to disinflation, despite the rise in unemployment and an external demand shock in 1995 that plunged Uruguay into a recession. This might have convinced the public to revise its inflationary expectations. The Phillips curve shifted downward in late 1996 and 1997, allowing unemployment to fall. With a second external demand shock since late 1998, unemployment has increased again. However, as confidence in the disinflation policies strengthened, Uruguay has obtained a much more favorable inflation-unemployment tradeoff than at the beginning of the decade.

The Phillips curve diagram in Figure 3 also suggests a distinction between structural and cyclical unemployment in Uruguay. The two (external) demand shocks of the 1990s have added a cyclical component to unemployment. Data on the duration of unemployment (Figure 4) support the notion that the deterioration on the labor market in the mid-1990s was in part cyclical. The graph shows a "duration loop" familiar from Western European economies. As economic conditions deteriorated (the "Tequila crisis"), employees were dismissed and entered

\footnotetext{
${ }^{1}$ Prepared by Benedikt Braumann.

${ }^{2}$ Because of its even income distribution and high level of social spending, Uruguay is often compared to Western European countries. A first glance at the labor market seems to support this comparison.
} 
the unemployment pool. The duration of an average unemployment spell fell, since the job losses were recent (1995). As the downturn bottomed out, unemployment continued to increase, and duration did so, too (1996). Job creation resumed with the start of a new recovery (1997-98) and the unemployment rate began to fall. However, firms first hired people with a short history of unemployment, since they appear more productive. The share of long-term unemployed and parallel to it the average duration increased. Only in a prolonged recovery will the long-term unemployed find jobs as well, and duration fall will (as happened in 1988-93).

A more precise measure of structural and cyclical unemployment can be obtained from a simple econometric estimation. The NAIRU (non-accelerating inflation rate of unemployment) is often used as an empirical approximation to structural unemployment. Figure 5 shows an estimate of the NAIRU in Uruguay. ${ }^{3}$ The unemployment rate is regressed on the change in CPI (DI) inflation (augmented Phillips curve) and a vector of structural variables, using monthly data over the period $1985.01-1999.03$. The structural variables include: real wages to account for union wage pressure (WR), cement sales for capital formation (CE), electricity consumption for industrial and household activity (EL), the relative price of non-tradables to tradables as a proxy for structural change (RP), real M2 balances (M2) and the slaughter of cattle (SL). An OLS regression yields the following:

$$
\begin{aligned}
& \mathrm{U}=20.8-0.075 * \mathrm{DI}+0.20 * \mathrm{WR}-4.71 * \mathrm{CE}-0.29 * \mathrm{EL}+0.075 * \mathrm{RP}-0.029 * \mathrm{M} 2+0.38 * \mathrm{SL} \\
& \quad(10.80)(5.46) \\
& (6.19)
\end{aligned}
$$

Adjusted $\mathrm{R}^{2}=0.67$, numbers in brackets are t-values.

This equation can be used to calculated the NAIRU by setting the change in inflation to zero and solving for unemployment.

Figure 5 shows that unemployment in Uruguay is mostly structural. Cyclical fluctuations around the NAIRU become more pronounced in the mid- and late 1990s, but they remain small compared to the overall level of unemployment. The NAIRU reached a peak during the crisis of 1983-85, and again during the years of high inflation 1989/90 before declining to just above 8 percent in the early 1990s. Structural change associated with the opening of the economy resulted in higher mismatch on the labor market and increased the NAIRU substantially in the mid-1990s.

${ }^{3}$ The calculation follows the method of Fallon, R. and Very, P. (1988): The Economics of the Labor Market. Oxford: Phillip Allan. 
Unemployment benefits, demographics, skills mismatch and real wage pressure from the unions are often cited as important reasons for high structural unemployment. These factors will be examined next for the case of Uruguay.

Uruguayan unemployment benefits are generous compared to the region, but the coverage is limited to workers in industry, trade and construction. ${ }^{4}$ Important sectors such as banking, agriculture and government are not included, but dismissals in these sectors are rare. Benefits are equivalent to 50 percent of the last wage and are disbursed for a maximum of six months. The data do not allow to calculate an average replacement ratio (average ratio of benefits to wages). However, the small number of people receiving benefits suggests that it might be quite low: In December 1994, 20,058 people, or less than 15 percent of all unemployed, were receiving benefits.

Unemployment is largely a phenomenon of second wage earners. Unemployment is relatively low among household heads (jefes de hogar). With an average rate of 3.5 percent over the last 12 years, this important group - at least until recently - enjoyed full employment (Figure 6). The incidence of unemployment is concentrated among women and the young, especially if they are not heads of a household. In March 1999, the unemployment rate for young people (below 25) was 30 percent, compared to 7 percent for older people. In both groups, women were about twice as likely to be unemployed as men. About 60 percent of all unemployed were people that did not lead a household.

About half of the job-seekers are not willing to take a job unless certain conditions are met. Figure 7 shows the requirements that the unemployed demand from their next job. The most important is to work in the same profession as before, which indicates a low inter-sectoral mobility of labor. High standards of education endow Uruguayan employees with significant amounts of specialized human capital. This makes the work force more productive but also, in the Uruguayan context, creates job specific expectations for employees, which makes the labor market vulnerable to sectoral restructuring.

On the other hand, the share of persons demanding no conditions from their prospective job has risen substantially, from 30 percent in 1993 to almost 60 percent in early 1999. This is indicative of increasing skill mismatch on the Uruguayan labor market after almost a decade of deep structural changes. The doubling of the unemployment rate for jefes de hogar points in the same direction (see Figure 6). Most of the increase of unemployment in the 1990s can be explained by this component.

\footnotetext{
${ }^{4}$ The following paragraph is based on ILO (1997): "Uruguay: Unemployment Insurance and Employment Services”, International Review of Social Security, 121-127.
} 
Figure 8 shows a historical time series of real wages in Uruguay. Unions were banned and collective bargaining suspended after the military took over the government in 1973. Firms were relatively free to adjust wages and employment for a period of 12 years. Upon the return to democracy in 1985, unions quickly re-organized and collecțive bargaining was restored. ${ }^{5}$ Real wages fell by about 50 percent during the military government, then stabilized after 1985, but hardly increased. This might reflect the fact that union density has been declining again after an initial surge. In 1987, 23 percent of all employees had become union members, but their number fell to 17 percent in 1993. In addition, most Uruguayan markets were opened up in the years 1973-85, and liberalized further during the 1990s. The labor market thus continued to be exposed to strong competitive forces.

In recent years, moderate wage increases have been accompanied by strong gains in productivity (Figure 9). Real wages grew by an average of only 0.5 percent since 1990 , compared with 4 percent for economy-wide productivity and 7.5 percent for productivity in manufacturing. This reduced unit labor costs and allowed for the net creation of almost 200,000 jobs in the economy since 1990 (Figure 10). Employment expanded by an average of 1.6 percent a year over this period. However, the increase in labor demand did not lead to lower unemployment as it was matched or even surpassed by an increase in labor supply. The participation rate increased from 58 to 63 percent over the 1990 s, mostly due to an increased participation of women. Thanks to this, the average Uruguayan household was able to increase its real income by about 20 percent, despite the small gains in real wages (Figure 11).

To sum up, most Uruguayan unemployment seems to be structural. A cyclical component of unemployment has emerged now and then in recent years, typically associated with negative external demand shocks. The main reasons for high structural unemployment include high reservation wages among second wage earners, structural change of the economy and a strong increase in labor supply. Real wage growth has been moderate over the 1990s. A more competitive environment and falling union density are gradually reducing unit labor costs. This has allowed the net creation of jobs and particularly a rising labor force participation of women. The addition of second wage earners contributed to an increase in real household income.

\footnotetext{
${ }^{5}$ See Allen, S., Cassoni, A., and Labadie, G. (1996): Wages and Unemployment after Reunionization in Uruguay. Cuadernos de Economia, 33, 277-293 for background and econometric evidence.
} 
Data Sources:

Figure 1. Latest national central bank bulletins.

Figure 2. International Labor Office (ILO): Labor statistics and National Statistical Institute (INE).

Figure 3. INE, Central Bank of Uruguay (BCU) and Fund staff calculations.

Figure 4. INE.

Figure 5. INE.

Figure 6. INE.

Figure 7. International Financial Statistics and INE.

Figure 8. ILO Labor statistics and BCU.

Figure 9. BCU and Fund staff calculations.

Figure 10. BCU.

Figure 11. BCU and INE. 
Figure 1. Historical Unemployment Rate

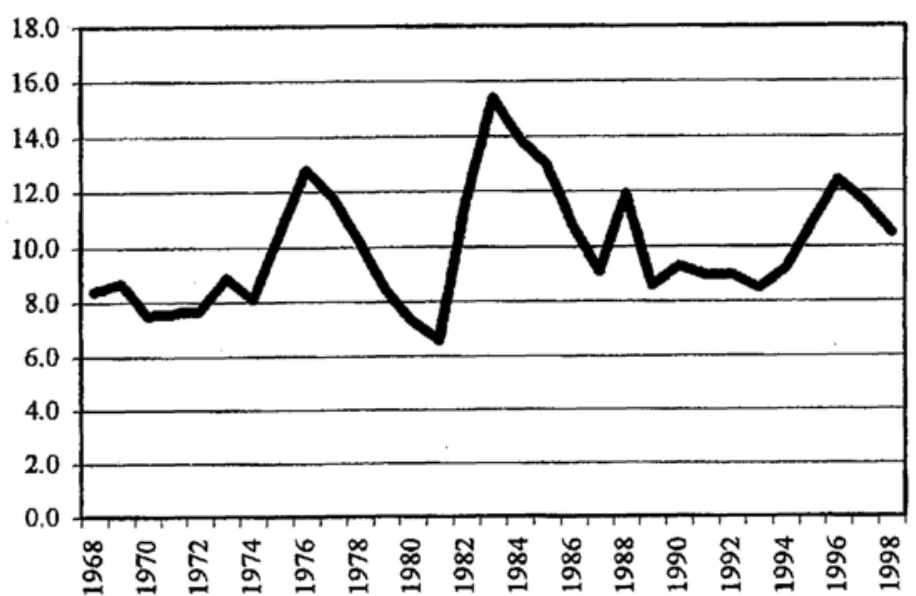

Figure 3. Phillips Curves 1985-99

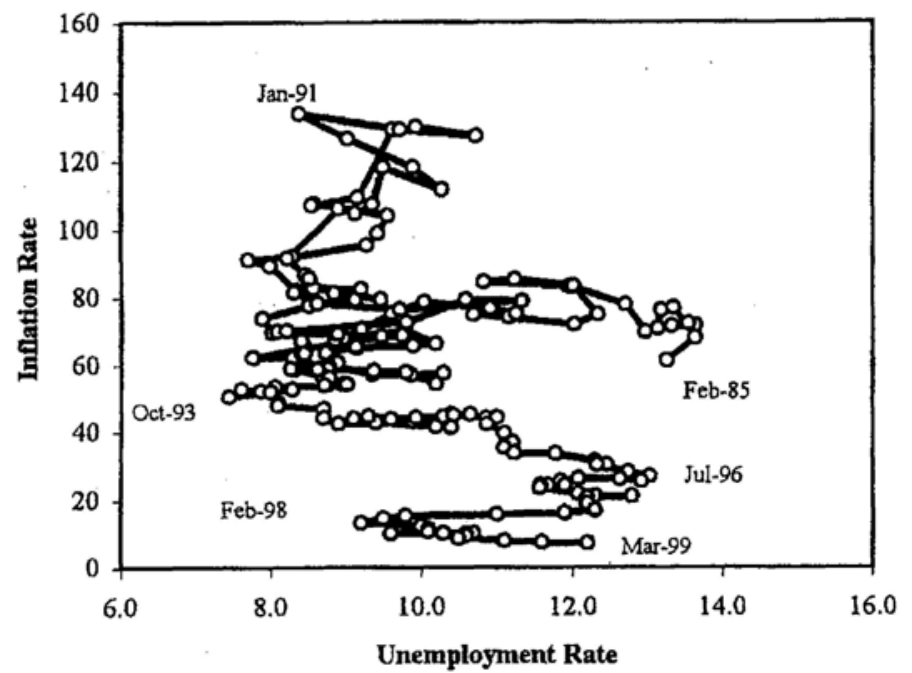

Figure 2. Unemployment in the Region - 1998

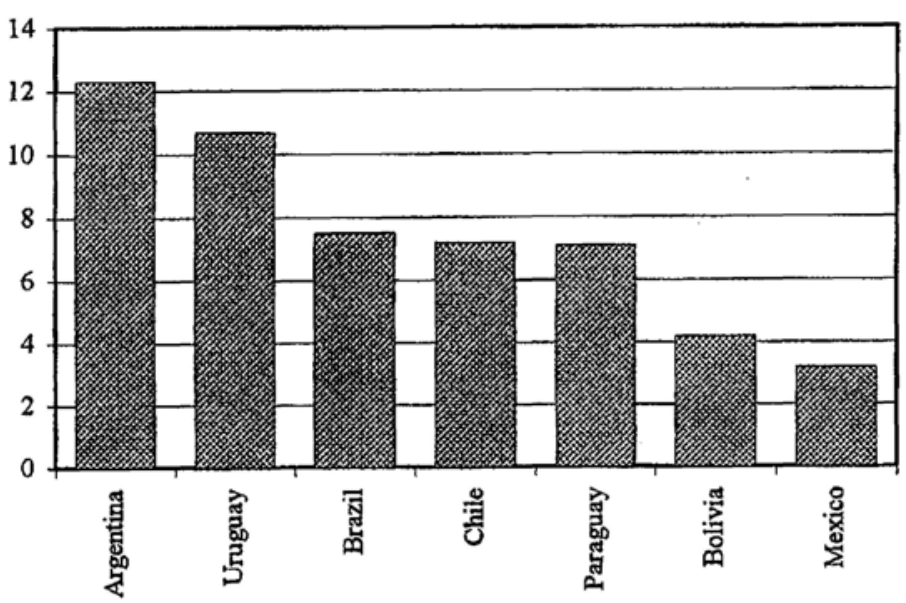

Figure 4. Duration of Unemployment

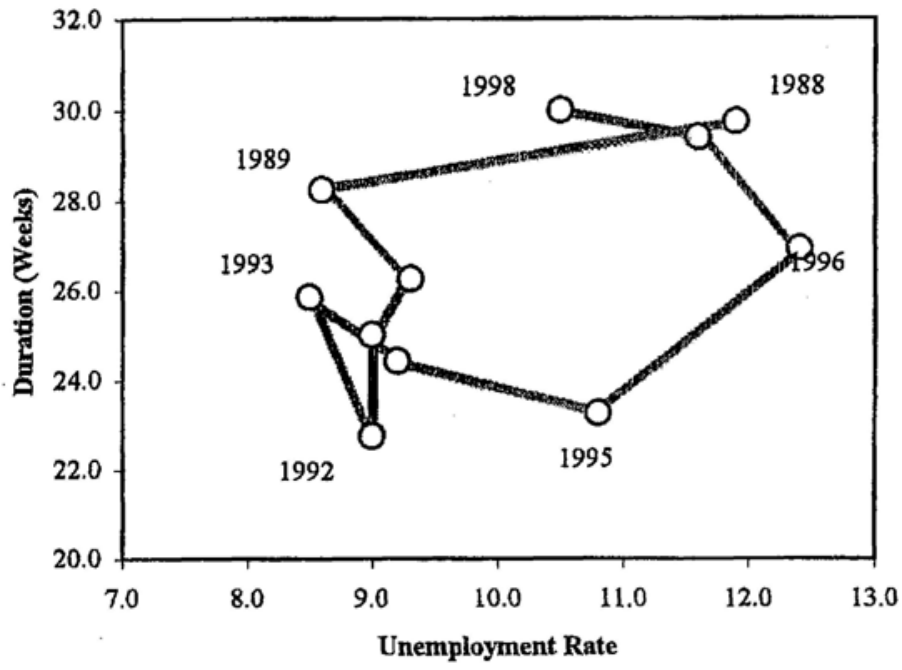




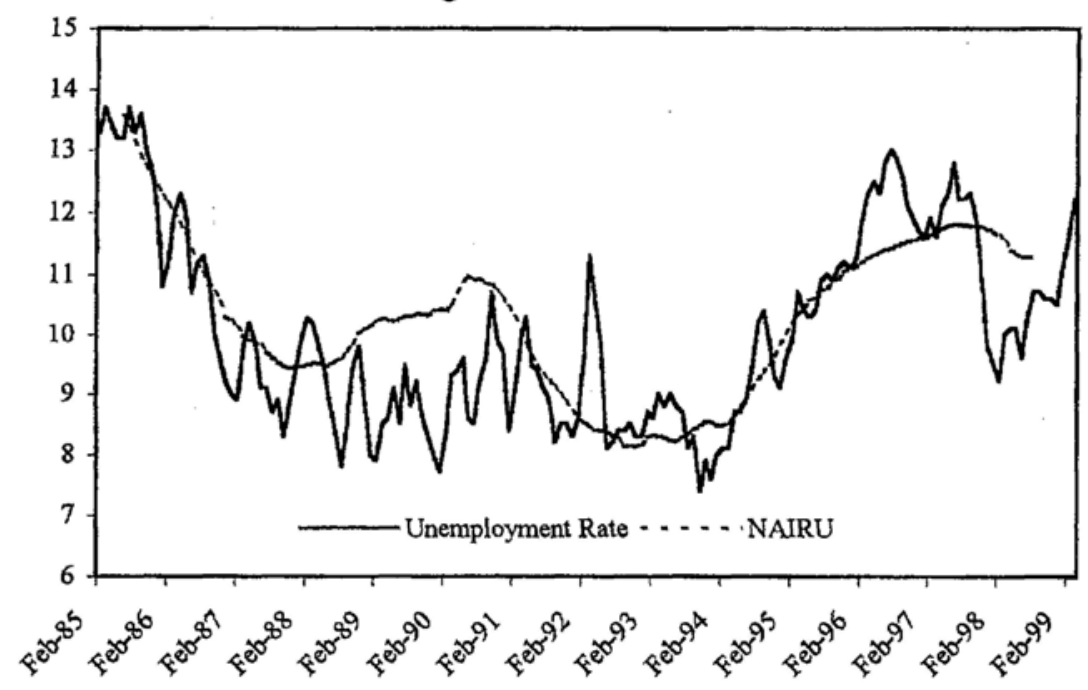

Figure 6. Unemployment Rates

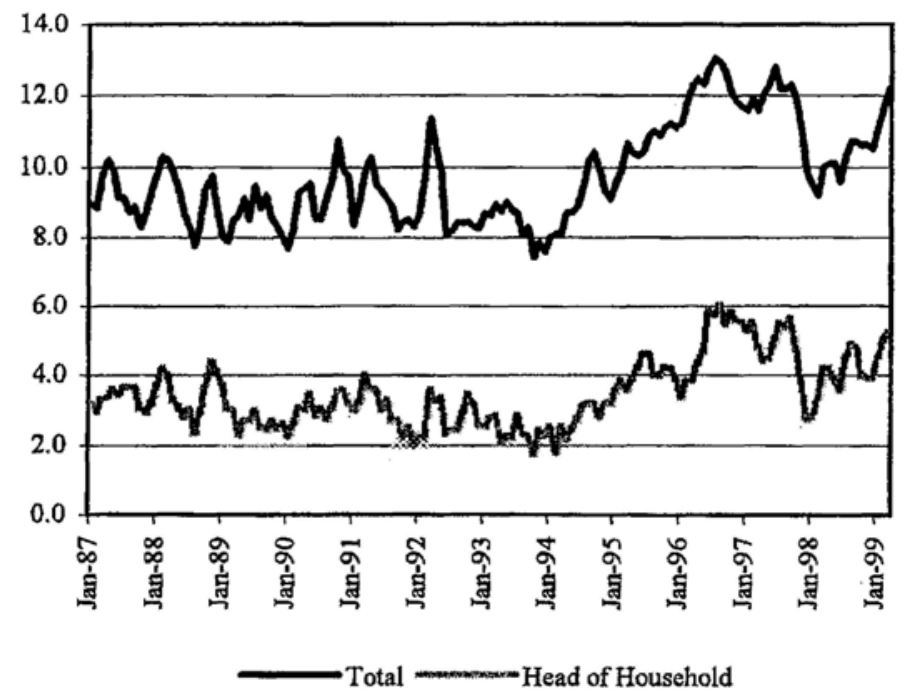

Figure 7. Requirements of Job-Seekers

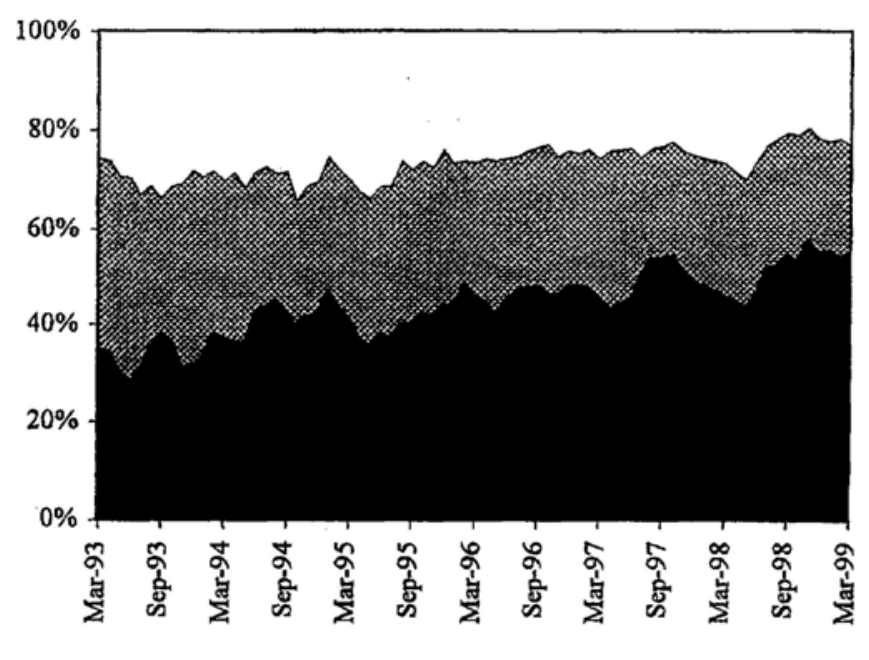




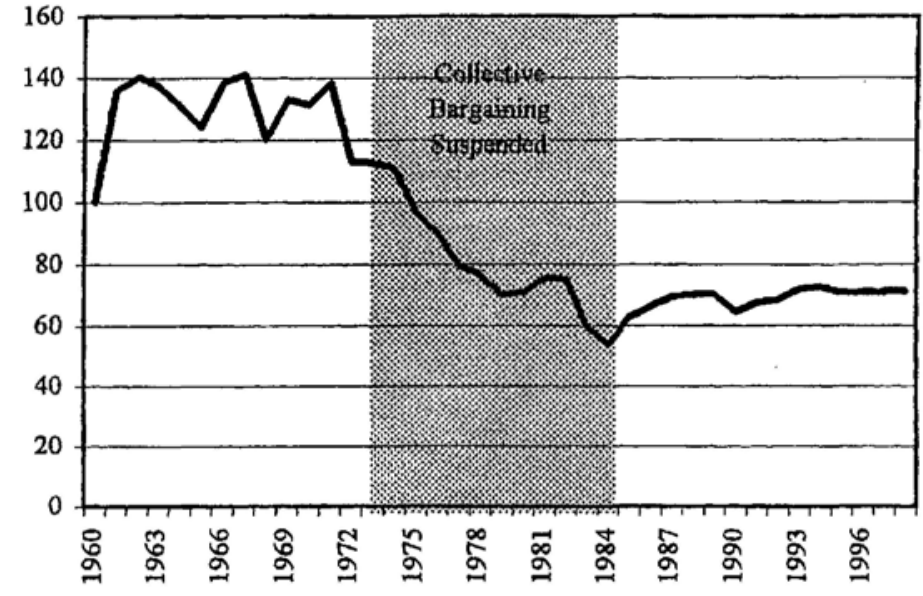

Figure 10. Total Employment

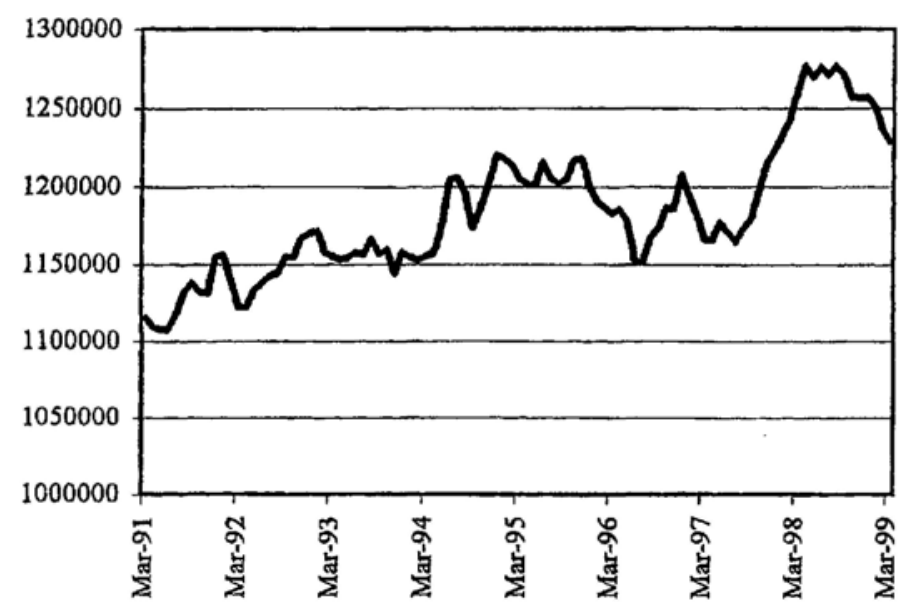

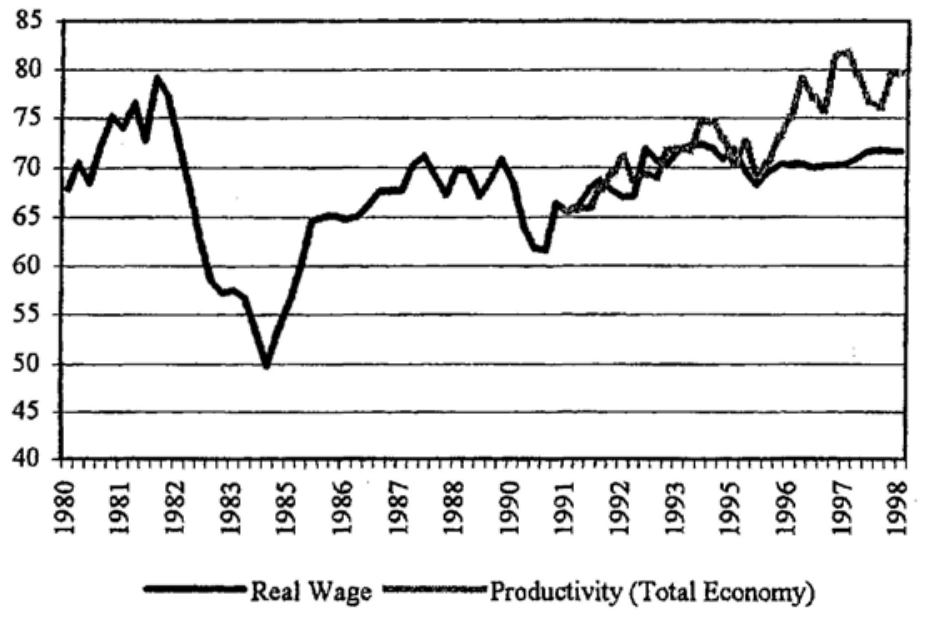

Figure 11. Real Wages and Household Income

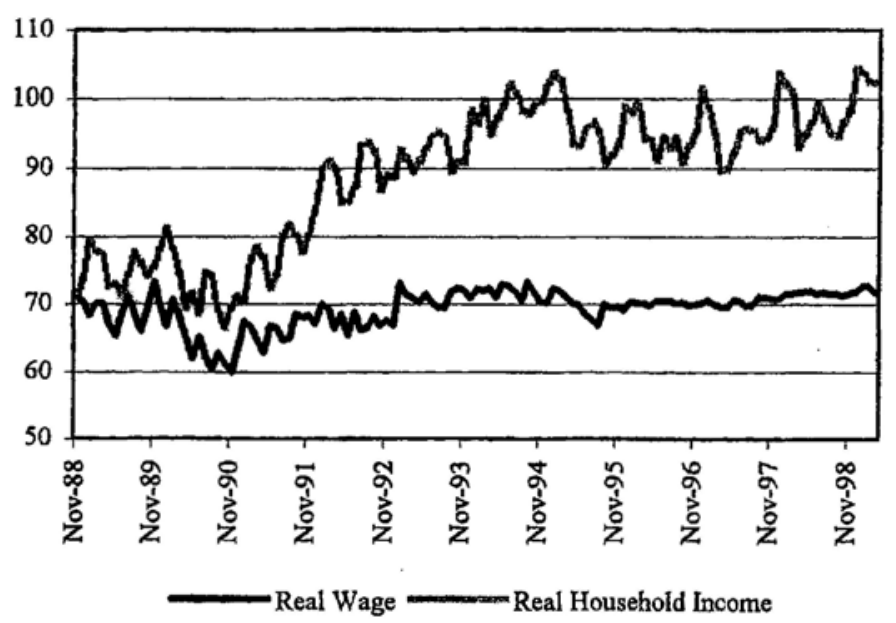




\section{INTERNATIONAL ReSERVES AdEQUACY In URUGUAY}

\section{Alternative Perspectives ${ }^{6 /}$}

This note examines Uruguay's gross international reserve holdings (including gold), in comparison to several other countries. The conclusions are:

- Measured against imports of goods and services, Uruguay's reserve holdings compare favorably with most other countries.

- This policy substantially insulates Uruguay against shocks to the current account and has benefited the country by increasing investor confidence and reducing interest rates.

- However, Uruguay is less well insulated against capital account shocks, according to ratios of international reserves to broad money, debt service, or the stock of short-term debt.

- Given that international reserves provide confidence, help to improve credit ratings, and lower interest spreads, if Uruguay were to accumulate additional reserves, and keep them permanently, the benefits of doing so would exceed the costs.

\section{A. Uruguay Has Shielded itself Well Against Shocks to Goods Markets}

As a traditional measure of a country's insulation against shocks to goods markets, international reserves are scaled by imports of goods and services. According to this measure, Uruguayan reserves have risen from under five months during the early 1990s to almost seven months (about US $\$ 2.6$ billion dollars) by end-1998. Also, by this measure, relative to other countries, Uruguay compares favorably. As Part A of Table 57 shows, this ratio for Uruguay substantially exceeds the mean for a sample of 19 selected emerging market countries.

\section{B. Uruguay's High Reserve Holdings Have Already Brought Benefits}

All else equal, increased holdings of international reserves should reduce country risk, increase investor confidence, and thus reduce interest rates. This hypothesis is supported by data on reserve levels and dollar interest spreads (relative to U.S. Treasury bonds) in several developing countries for which such interest rates are available, including Uruguay, as presented in Table 58.

Many factors determine a country's spread over U.S. interest rates. However, even without examining such other factors, these data suggest that lower levels of international reserves are generally associated with a higher yield spread paid on their international bond issues. A simple bivariate regression yields an elasticity of the spread with respect to the reserves to import ratio of approximately minus one half. That is, a 1 percent increase in reserves (measured in months of

${ }^{6}$ Prepared by Jose Bailen, Juan Carlos Jaramillo, and Evan Tanner. 
imports) will reduce the spread (measured in basis points) by $1 / 2$ percent. Moreover, the only two countries that paid a lower interest spread than Uruguay in recent bond issues, Chile and China, also held more reserves (relative to imports) than Uruguay; and except for Argentina, all countries that pay a higher interest spread than Uruguay also hold fewer reserves (relative to imports).

\section{However, it is Difficult for Countries to Fully Protect Themselves from Shocks to the Capital Account}

Another motive for international reserve holdings should be to protect a country from financial shocks like market contagion and deposit outflows, while also permitting external debt obligations (both short- and long- term) to be serviced.

The ratio of official reserves to currency indicates the monetary authorities' ability to withstand pressure on the exchange rate stemming from a flight away from the local currency (but not away from banks). As the data in Part B of Table 57 suggest, Uruguay's official reserves are about twice as large as currency, similar to the average observed in other emerging economies.

A broader indicator of a country's ability to confront a flight from its liquid assets may be gauged by its ratio of official reserves to broad money (the sum of money and quasi-money). As the data in Part $\mathrm{C}$ of Table 57 suggest, by this measure, Uruguay compares less favorably against an average of 23 emerging market economies. The average for these countries has increased in recent years, from about 39 percent in 1990 to over 52 percent by end 1998. In Uruguay, this ratio grew rapidly, from 16.2 percent in 1990 to 24.2 percent in 1998. Despite this rapid growth, however, it remains below the average of other emerging economies.

The above calculation treats all countries alike and thus ignores Uruguay's unusual position as a safe haven for deposits. In Uruguay, such nonresident deposits are an important component of broad money and are not typically intermediated domestically. Instead, the banks hold the counterpart of these deposits themselves in relatively liquid foreign assets. At end-1998, liquid foreign assets roughly offset such deposits in Uruguayan banks. If these deposits are excluded from broad money, the ratio of official reserves to broad money rises to about 37 percent. Even considering this adjustment, however, this ratio for Uruguay remains below the average for other emerging economies.

Scheduled debt service (both short- and long- term) also plays a role in evaluating reserve holdings. In a crisis, debt service will strain reserves, as debt rollover or new credits will be difficult to obtain. Greater reserve holdings should, all else equal, facilitate repayment and reduce difficulties to renew old debts.

As Part D of Table 57 shows, Uruguay's coverage of official reserves to debt service (which excludes amortizations on short-term debt) has risen dramatically in recent years, from 39 percent in 1990 to over 160 percent in 1998. This exceeds the average for the Western Hemisphere countries, but nonetheless is below that for other comparable net debtor countries. 
Part E of Table 57 also shows the ratio of official reserves to short-term external debt for several groups of countries and for Uruguay. Uruguay's ratio has remained around 80 percent and is above the Latin American average, but it is somewhat below the reserve coverage of short-term debt of other comparator emerging-market countries, whose ratio exceeds 90 percent.

\section{For Uruguay, the Eventual Benefits of Additional Reserve Holdings Exceed the Costs}

Reserve holdings imply a cost. For debtor countries, the opportunity cost of reserves is best measured by the difference between the interest rate paid on external debt and that received on reserve assets. In 1998 the implicit average interest rate paid on the external debt was 6.6 percent. At the same time, the implicit rate of return of nongold reserve assets was 4.5 percent. ${ }^{7}$ Thus, opportunity cost of holding reserves is about 2 percent. As mentioned above, there is evidence that an increase in reserve holdings benefit a country by lowering interest rates it faces in world markets.

To compare costs and benefits, consider a permanent increase in reserve holdings of US $\$ 100$ million. The yearly, undiscounted cost of such additional reserve holdings will be US\$2.1 million, while the ratio of reserves to imports would increase by 4 percent. The evidence presented in Section 2 suggests that the spread (measured in basis points) would fall by about 2 percent. Recently, the spread faced by Uruguay has been about 300 basis points. ${ }^{8}$ Thus, the spread should fall by about six basis points.

The benefits of additional reserve holdings depend on whether the new, lower spreads apply to only new, additional debt or to the entire existing debt as well. In the less favorable case, suppose that the new, lower spread applies only to short term debt, about US $\$ 5$ billion. In this case, the immediate benefit to Uruguay would be about US\$3 million, greater than the cost of US\$2.1 million mentioned above.

However, if the reduction in spreads is permanent, the gains will also apply to the medium-and long- term debt (about US\$7 billion dollars) as it is rolled over. In undiscounted terms, yearly benefits on the total debt stock (short, medium, and long term) would be about US\$7.2 million (compared to costs of US\$2.1 million).

\footnotetext{
${ }^{7}$ As an extreme example, if Uruguay were to use its total stock of reserves (averaging about US $\$ 2.4$ billion in 1998) to retire foreign debt, it would save about US $\$ 49$ million, or 0.2 percent of GDP.
}

${ }^{8}$ This figure is somewhat less than that in Table 58, reflecting recent developments. 
Table 57. Uruguay: Indicators of International Reserves Adequacy

(In percent)

1990

1995

1996

1997

1998

A. Ratio of official reserves to imports of goods and services

19 emerging market economies (group average)1/

2.9

4.1

4.4

3.9

4.8

Uruguay

4.6

6.1

5.8

5.7

6.9

B. Ratio of official reserves to currency outside banks

19 emerging market economies (group average)1/

Uruguay

$\begin{array}{lllll}146.4 & 283.1 & 314.7 & 287.0 & 287.0 \\ 179.1 & 181.9 & 202.6 & 237.3 & 287.4\end{array}$

C. Ratio of official reserves to money plus quasi-money

19 emerging market economies (group average)1/

Uruguay

Uruguay (adjusted 2/)

$\begin{array}{lllll}42.6 & 53.2 & 53.4 & 57.8 & 59.7 \\ 16.2 & 19.1 & 18.5 & 20.3 & 24.2 \\ 34.0 & 28.3 & 28.2 & 31.3 & 37.2\end{array}$

D. Ratio of gross official reserves to total debt service

$\begin{array}{lrrrrr}19 \text { emerging market economies (group average)1/ } & 103.8 & 173.5 & 176.3 & 147.8 & 139.7 \\ & & & & & \\ \text { Net debtor countries } & 116.4 & 183.3 & 200.1 & 204.2 & 188.0 \\ \text { Developing countries } & 133.8 & 190.9 & 206.8 & 212.9 & 196.4 \\ \text { Western Hemisphere } & 77.6 & 132.2 & 136.8 & 120.9 & 112.8 \\ \text { Uruguay } & 39.2 & 91.2 & 95.7 & 148.4 & 161.3\end{array}$

E. Ratio of gross official reserves to short term external debt

19 emerging market economies (group average)1/

$6.8-107.6$

102.4

91.4

Net debtor countries

$\begin{array}{lllll}113.9 & 129.0 & 136.0 & 138.4 & 130.1\end{array}$

Developing countries

$\begin{array}{lll}123.6 & 134.8 \quad 141.3\end{array}$

$143.8 \quad 134.8$

Western Hemisphere

$\begin{array}{lll}83.5 & 93.8 & 98.6\end{array}$

93.1

73.0

Uruguay

n.a. $\quad 77.1$

82.3

88.6

79.6

Sources: IFS; WEO Database; Central Bank of Uruguay; and Fund staff estimates.

1/ Includes Argentina, Bolivia, Brazil, Chile, Colombia, Dominican Republic, Ecuador, Egypt, Guatemala, Malaysia, Mexico, Panama, Paraguay, Peru, Thailand, Trinidad and Tobago, Turkey, Uruguay, and Venezuela.

2/ Ratio estimated with money plus quasi-money excluding nonresidents' deposits. 
Table 58. Uruguay: International Reserves and Yield Spread by Emerging Markets

\begin{tabular}{lrrrr}
\hline & \multicolumn{2}{c}{1997} & & \multicolumn{2}{c}{ Q4 1998-Q1 1999 } \\
\cline { 2 - 5 } & $\begin{array}{r}\text { Reserves/month } \\
\text { of Imports 1/ }\end{array}$ & $\begin{array}{r}\text { Yield } \\
\text { Spread }\end{array}$ & $\begin{array}{r}\text { Reserves/month } \\
\text { of Imports 1/ }\end{array}$ & $\begin{array}{r}\text { Yield } \\
\text { Spread }\end{array}$ \\
\hline Argentina & 8.8 & 410 & 9.1 & 711 \\
Chile & 10.6 & 124 & 9.4 & 289 \\
Colombia & 7.4 & 162 & 5.9 & 667 \\
Mexico & 4.5 & 247 & 4.5 & 574 \\
Panama & 6.9 & 239 & 3.3 & 417 \\
Uruguay & 5.3 & 137 & 6.5 & 357 \\
China & 12.1 & 92 & 12.3 & 292 \\
Philippines & 2.3 & 231 & 3.4 & 419 \\
\hline
\end{tabular}

Sources: IFS, April 1999 and Private Market Financing for Emerging Markets, Q1 1999.

1/ Gold valued at SDR 35 per ounce. 